\title{
Diamond Photonics for Distributed Quantum Networks
}

\author{
Sam Johnson, Philip R. Dolan and Jason M. Smith \\ Department of Materials, University of Oxford, Parks Road, Oxford, OX1 3PH
}

\begin{abstract}
The distributed quantum network, in which nodes comprising small but well-controlled quantum states are entangled via photonic channels, has in recent years emerged as a strategy for delivering a range of quantum technologies including secure communications, enhanced sensing and scalable quantum computing. Colour centres in diamond are amongst the most promising candidates for nodes fabricated in the solid-state, offering potential for large scale production and for chip-scale integrated devices. In this review we consider the progress made and the remaining challenges in developing diamond-based nodes for quantum networks. We focus on the nitrogen-vacancy and silicon-vacancy colour centres, which have demonstrated many of the necessary attributes for these applications. We focus in particular on the use of waveguides and other photonic microstructures for increasing the efficiency with which photons emitted from these colour centres can be coupled into a network, and the use of microcavities for increasing the fraction of photons emitted that are suitable for generating entanglement between nodes.
\end{abstract}

Keywords: Diamond photonics, Quantum networks, Color centers, Nitrogen-vacancy, Silicon-vacancy, Microcavities

\section{Contents}

1 Introduction $\quad 2$

1.1 Quantum Networks . . . . . . . . . . . . . . . . . . . 2

1.2 Considerations and Requirements for Nodes in Quantum Networks . . . . . . . . 3

2 Colour centres in diamond as networkable spin qubits 6

2.1 The Nitrogen-Vacancy Centre . . . . . . . . . . . . . . . . . . . 6

2.2 The Silicon-Vacancy Centre . . . . . . . . . . . . . . . . . 13

2.3 Emerging Colour Centres . . . . . . . . . . . . . . . . . 16

3 Engineering Diamond for Nodes in Quantum Networks 16

3.1 Fabrication and Processing . . . . . . . . . . . . . . . . . 16

3.1 .1 Reactive Ion Etching . . . . . . . . . . . . . . . 17

3.1 .2 FIB Milling . . . . . . . . . . . . . . . . . . . . . . . . . . . . . 18

3.2 Generation and Placement of Defects . . . . . . . . . . . . . . . . . 18

Email address: sam.johnson@materials.ox.ac.uk (Sam Johnson, Philip R. Dolan and Jason M. Smith) 
3.2.1 Ion Implantation $\ldots \ldots \ldots \ldots \ldots$

3.2.2 Vacancy Generation . . . . . . . . . . . . . . . . . . . . 21

3.2.3 Delta-Doping for Colour Centre Positioning . . . . . . . . . . . . . 22

3.3 Diamond Surface Preparation . . . . . . . . . . . . . . . . . . . 23

3.4 Coherence Properties of Colour Centres in Engineered Diamond . . . . . . . . 23

4 Photonic Structures I: Fluorescence Collection and Waveguiding 26

4.1 Solid Immersion Lenses _ . . . . . . . . . . . . . . . . . . . . 26

4.2 Bullseye Structures . . . . . . . . . . . . . . . . . . . . . 27

4.3 Nanowires and Nanopillars . . . . . . . . . . . . . . . . . . . 27

4.4 On-Chip Waveguides and Photonic Elements . . . . . . . . . . . . . . 30

5 Photonic Structures II: Optical Resonators 31

5.1 Integrated Resonators . . . . . . . . . . . . . . . . . . . . . . . . . . . 34

5.1.1 Photonic Crystal Cavities (PCCs) . . . . . . . . . . . . . . . . 34

5.1 .2 Whispering Gallery Resonators . . . . . . . . . . . . . . . . . . 39

5.1.3 Hybrid GaP/Diamond Devices . . . . . . . . . . . . . . . . . . . . . 39

5.2 Open Cavities . . . . . . . . . . . . . . . . . . . . . . . 42

5.2.1 Fabrication of Open Cavities . . . . . . . . . . . . . . . 42

5.2.2 Colour Centre Coupling to Open Cavities . . . . . . . . . . . . . . 44

6 Conclusions and Future Outlook $\quad 47$

7 Acknowledgments $\quad 48$

\section{Introduction}

\subsection{Quantum Networks}

The quantum network, consisting of discrete nodes that are linked by entanglement (Figure 1) has become popular in recent years as a route towards scalable quantum processors [1](Figure 1a), secure sensor networks and the 'quantum internet' [2]. Each node consists of a quantum bit (qubit) or multiple qubits that can be entangled together at will using deterministic and reversible local gate operations. Entanglement between nodes is generated by first entangling the nodes with photons and then performing measurements on the photons which project the nodes into an entangled state. The use of photonic channels provides flexibility in the physical scale of the network, from chip-based systems to larger networks utilising optical fibres or free-space line-of-sight pathways (Figure 1b). The fact that the nodes themselves are not required to interact directly with each other also provides opportunities for heterogeneity between different systems. Nodes might consist for example of ion trap systems, cold atoms, superconducting circuits, or solid state devices incorporating spins on defects or quantum dots. It is likely that over the coming years, as quantum technology matures, devices using these and other platforms will be developed to make use of the full range of emerging quantum functionality for different applications. Ultimately, the vision is for a 'quantum internet of things' in which quantum entanglement between devices provides capabilities for information gathering, security, communication and processing that exceed the limits of classical information systems.

This article reviews recent progress towards the realisation of quantum networks using diamond colour centre defects and photonic structures as nodes. Diamond has for a number of years 
(a)

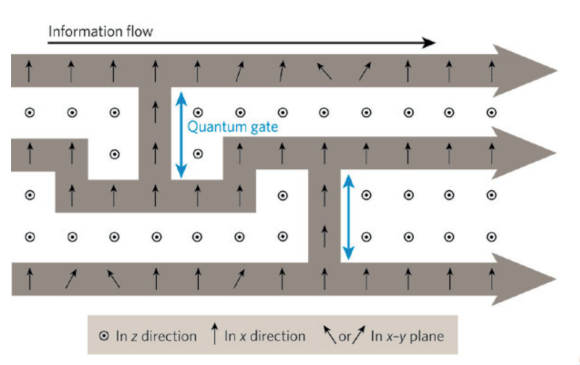

(b)

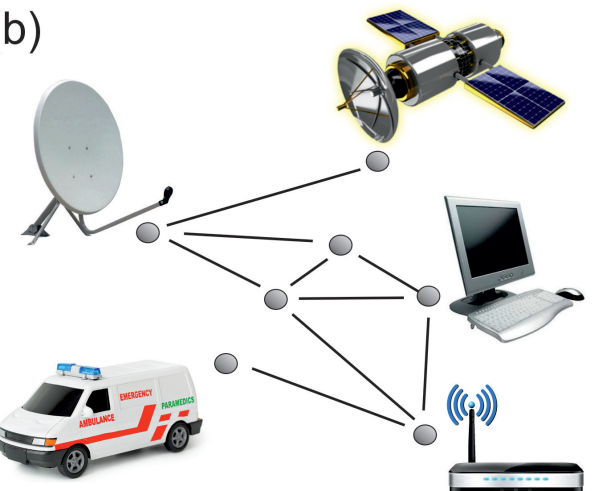

Figure 1: Quantum networks. (a) A network 'circuit' for scalable quantum computing. Reprinted by permission from Macmillan Publishers Ltd: Nature [3], copyright 2008. (b) A large heterogeneous network with different nodes linked by optical fibre and free-space channels.

been recognised as one of the most promising solid state platforms for networked quantum technologies [4]. Its combination of a nearly nuclear spin-free environment, high Debye temperature, and wide band gap, result in an extremely 'quiet' environment in which isolated electron spins can display coherence times of order a millisecond at room temperature [5], and approaching a second at cryogenic temperatures [6], with nuclear spins offering coherence times well in excess of a second [7,8]. These spin states can be initialised, manipulated, and measured with high fidelity [9] using optical, microwave and radio frequency control gates, thus fulfilling the key DiVincenzo criteria for qubits to be used in quantum computing [10]. Spin-dependent electric dipole transitions in colour centres provide an efficient optical interface with which to link the electron spin qubits to a photonic network. A 'node' of a network would therefore typically consist of a single colour centre defect with an electron spin coupled via the hyperfine interaction to the spins of nearby nuclei. The first demonstrations of simple two-node networks have in the past three years been reported by the Hanson group at Delft University [11, 12, 13] using negatively charged nitrogen-vacancy (NV) defects as nodes.

One of the principal attractions of diamond for quantum networks, in common with some other solid state systems, is its compatibility with integrated photonic approaches. This provides not only a means for fabricating on-chip networks, but for controlling the interface between network photons and the colour centre nodes using optical microresonators.

\subsection{Considerations and Requirements for Nodes in Quantum Networks}

Before embarking on the subject of diamond photonics for quantum networks, it is worthwhile to review briefly the methods that have been proposed for generating entanglement between discrete nodes, and the requirements they place on the architectures, components and methods to be used.

A key concept in the generation of entanglement between nodes by projective measurement of photons is that of 'path erasure', namely that when a measurement is performed it reveals information about the collective state of the nodes to be entangled, but not each node individually, an idea initially proposed in 1999 by Cabrillo et al [14]. In 2005, Barrett and Kok [15] showed how this concept could be used to generate scalable entangled networks by devising a simple 


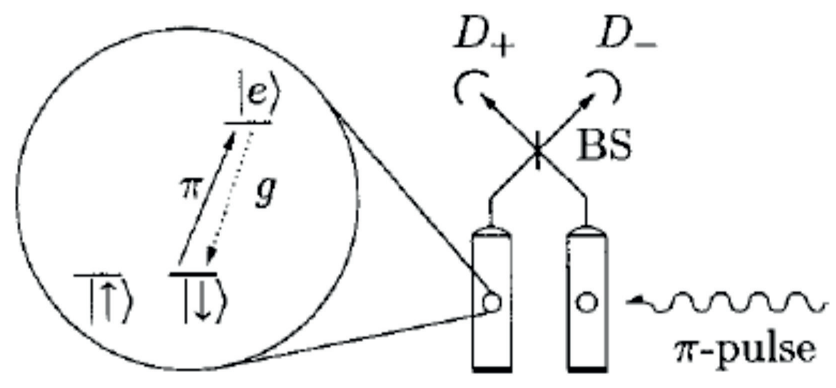

Figure 2: The Barrett \& Kok 'double heralding' scheme for creating maximally entangled states via a photonic network. Two physical qubits spontaneously emit photons conditionally on their spin state. The photons are interfered on a beam splitter before being directed to two detectors. A detection event at one of the detectors reveals the presence of a photon, but not from which node it originated - a technique known as 'path erasure'. Reprinted with permission from [15] Copyright 2005 by the American Physical Society.

protocol for producing a heralded, high fidelity Bell state between two qubits. In their scheme (figure 2) the quantum level structure within each node includes an optical transition (labelled $\pi)$ that is sensitive to the electron spin state $(|\uparrow\rangle,|\downarrow\rangle)$, such that after excitation the presence of a photon spontaneously emitted from a node $(g)$ provides information about that node's spin, in other words the spin state becomes entangled with the photon number at the node output. A measurement system is set up in which two nodes are measured simultaneously in such a way that detection of a photon on detector $D_{+}$or $D_{-}$reveals the encoded information but crucially does not reveal from which node it originated. The result of a photon detection event is that the combined state of the system necessarily collapses into the state that is consistent with the outcome of the measurement (the inconsistent terms are 'measured out'), which, with an appropriate choice of measurement, will be an entangled state of the two nodes. In this scheme the entanglement is imperfect after the first photon detection event. Both spins are then inverted and the measurement process repeated upon which detection of a second photon projects the spins into the maximally entangled Bell state. These photon detection events 'herald' the successful generation of entanglement, and so Barrett \& Kok and similar two-photon detection schemes, are known as 'double heralding' schemes. The successful implementations of this scheme in diamond will be discussed in more detail in section 2.1 .

The path erasure process requires that after each photon detection event there is no way of telling, even in principle, the origin of a measured photon. This places two quite stringent conditions on the measurement process: firstly that photons emitted from the two nodes must be formally indistinguishable such that they can be mapped onto the same quantum states, most commonly using a simple 50:50 beam splitter with two input and two output ports; and secondly that the nodes themselves must have no 'memory' of whether or not they were excited, for example by the presence of a lattice vibration (phonon). The condition of indistinguishability is a rather stringent constraint - the photons must be identical in all respects, including in their frequency distribution and the arrival time of the photon wave-packet at the detector. To fulfill these conditions without significantly compromising efficiency, the photons need to be 'transform-limited', meaning that the spectral shape and line-width of the photon are determined by the Fourier transform of the spontaneous emission decay function, i.e that the time envelope 
for the photon extends over the entire spontaneous emission lifetime. To achieve this condition requires an absence of decoherence on the timescale of the spontaneous emission process, a situation that in solid state environments only occurs in a few select systems and invariably requires cryogenic temperatures of a few Kelvin to suppress phonon interactions. To meet the condition of identical frequency distribution from different nodes may also require some tuning capability, generally using an electric field to generate a Stark shift in one or more of the optical transitions (section 2.1). The condition of 'no memory' requires that the optical excitation of the qubit be resonant with the emitted photon to ensure that no tell-tale energy is dispersed in the node as a result of the spin-dependent excitation.

As well as Barrett \& Kok, several other measurement-based entanglement schemes have been proposed that could be used to generate scalable quantum networks $[16,17,18,19,20]$. The details of these are beyond the scope of this review, but two by $\mathrm{Hu}$ et al [19] and Nemoto et al [20] are worth highlighting because they may serve to relax the constraints on the spontaneous emission process described above. Essentially in these two works the photons which carry the information, rather than being spontaneously emitted by the node, are scattered from it, and indistinguishability can therefore be established straightforwardly by using photons from a common coherent source. This brings the advantage that because scattering effects can be observed at frequencies detuned from the resonant transition, small differences in the optical transition energies of the two nodes, and increased dephasing in the optically excited state, can be accommodated. Finally we note recent theoretical work which shows that indistinguishable photons can be produced by strongly dephasing emitters coupled to high quality cavities [21], providing a further possible route towards nodes that can operate at higher temperatures.

A common feature of heralded entanglement-by-measurement schemes is that they are probabilistic and may need to be repeated multiple times before entanglement is successfully achieved. If large scale entangled states are to be created it is therefore important to preserve any existing entanglement links while attempting to create new ones. This can be achieved by a procedure known as 'brokering' [22] in which any entanglement successfully created on a 'broker' qubit is passed deterministically onto a protected neighbouring 'client' qubit before further entanglement operations commence. Diamond colour centres are well suited to brokering schemes: the electron spin to which the optical transition couples is the broker and a nearby nuclear spin, decoupled from the environment and from the optical signals, is the client. Recent work by the Delft group has shown that selected nuclear spin states can be preserved through multiple entanglement attempts with the electron spin [23].

Even with brokering schemes in place, the efficiency of the entanglement process is a critical parameter in determining the size of entangled network that can be created. In doubleheralding pairwise entanglement schemes such as Barrett \& Kok, the entanglement efficiency scales quadratically with the efficiency $\eta$ of detecting a single photon from one of the nodes at $D_{+}$or $D_{-}$. This probability can itself be expressed as the product of several factors:

$$
\eta=\eta_{e x} . \eta_{e m} . \eta_{c o} . \eta_{t r} . \eta_{d e t}
$$

where $\eta_{e x}$ is the probability of excitation, $\eta_{e m}$ is the probability of emission of a suitable photon, $\eta_{c o}$ is the probability that the photon will be collected into the network channel, $\eta_{t r}$ is the probability that it will be transmitted to the detector, and $\eta_{d e t}$ is the quantum efficiency of the single photon detector. To bring all of these factors close to unity is a major challenge: for example $\eta_{e x}$ is reduced by instabilities in the node resonance resulting in detuning from the excitation laser; $\eta_{e m}$ is limited by the branching ratio of the emitter; $\eta_{c o}$ is only a few percent without the 
use of optical microstructures or resonators; and $\eta_{t r}$ can be severely limited by attenuation in the network channels, particularly in long range networks where scattering and absorption of photons may be considerable. In the Delft experiments to date, the efficiency of detection of single indistinguishable photons has been between $10^{-4}$ and $10^{-3}$, yielding an entanglement probability per attempt of order $10^{-7}$. Development of nodes that facilitate improvement of these figures is now the focus of considerable effort by many research groups.

For scalable quantum computing, the concept of 'fault tolerance' is critical in determining the degree to which architectures can withstand errors in individual components. In particular the fidelity of the entanglement created, along with the subsequent measurements and control gates, will have a threshold below which scalability is not possible. This fidelity threshold is highly dependent on the computing protocol to be used, and considerable effort is focused on developing intrinsically fault-tolerant protocols. In recent work, scalable computing has been shown to be possible with threshold node fidelity around $99 \%$, even if the network itself is noisy and lossy, using entanglement distillation [24]. Such performance has been achieved using NV defects in diamond [25], and is assisted by the facility for local error correction [26, 27, 28]. Entanglement distillation and error correction rely upon local operations acting on individual nodes, and so do not place requirements on the photonic subsystem or network, beyond the need for sufficient number of qubits in each node to perform the operations (five in the case of entanglement distillation [24]) and the integration of the local control systems required for spin manipulation.

\section{Colour centres in diamond as networkable spin qubits}

Diamond plays host to hundreds of optically active point defects or colour centres, with emission from the blue to the near infra-red region of the spectrum [29,30]. Only a subset have been identified and studied at the single defect level and of these, the nitrogen-vacancy centre and silicon-vacancy $(\mathrm{SiV})$ centre, have emerged as potential nodes for quantum networks. The following section will present an introduction to these defects and their enabling features for this application. The understanding and implementation of the $\mathrm{NV}$ centre is at a more mature stage, allowing milestone demonstrations of spin-photon [31] and remote spin-spin entanglement $[11,12,13]$ which are discussed in section 2.1 . The $\mathrm{SiV}$ has more recently been identified as having potential for use in quantum networks, in particular due to some attractive optical properties. This progress will be reviewed in section 2.2 , before a brief discussion of alternative defects.

\subsection{The Nitrogen-Vacancy Centre}

The negatively charged $\mathrm{NV}$ centre $\left(\mathrm{NV}^{-}\right)$, illustrated in figure 3 , is the most widely studied colour centre in diamond, and besides its potential for quantum networks, it has attracted widespread interest for use as a nanoscale sensor in the physical [32] and biological [33] sciences. Consisting of a lattice vacancy bound to a substitutional nitrogen along the [111] bond axis (figure 3(b)), the six valence electrons of $\mathrm{NV}^{-}$form a spin triplet ground state that provides a natural long-lived qubit with long spin coherence time. The spin-spin interaction raises the energy of the $\mathrm{m}_{s}= \pm 1$ states relative to $\mathrm{m}_{s}=0$ by $2.88 \mathrm{GHz}$, such that magnetic dipole transitions between these states can be driven coherently via a simple antenna using standard microwave electronics. The ground state couples to an orbitally degenerate excited state triplet manifold via spin-dependent optical transitions, and intersystem crossing to a pair of spin singlet 


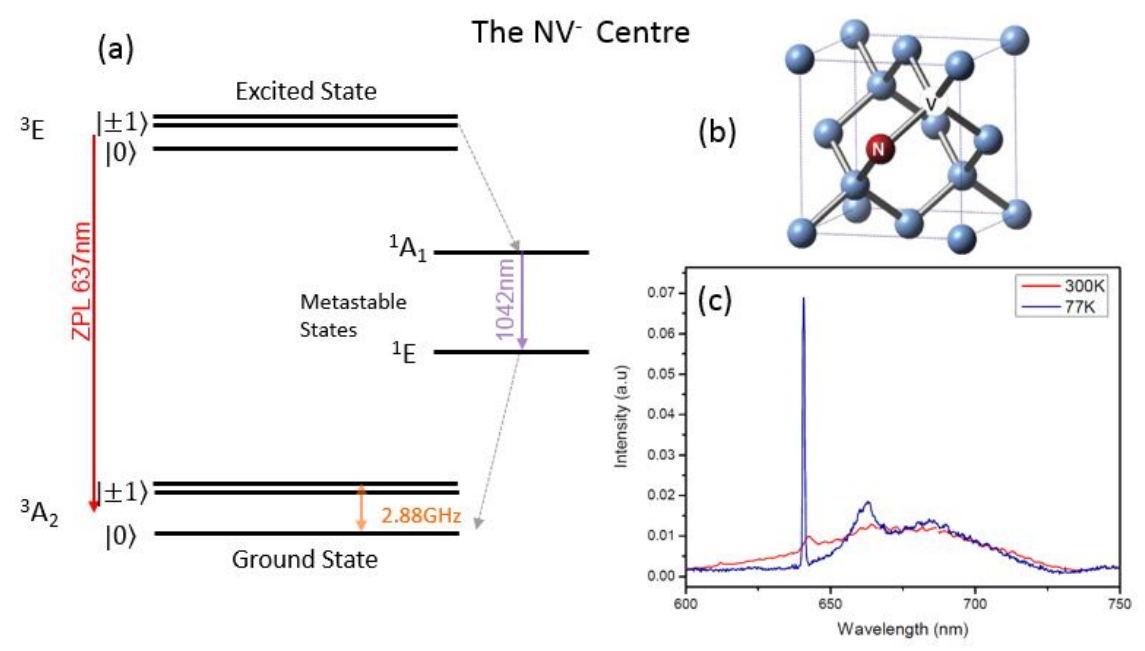

Figure 3: The $\mathrm{NV}^{-}$centre in diamond. (a) The basic energy level structure. The crystal field splits the triplet sub-levels of the ground and excited state. The zero-phonon line (ZPL) transition is indicated. The dashed arrows indicate the dominant transitions to and from the metastable shelving states which enable the optical spin polarisation mechanism. (b) A substitutional nitrogen $(\mathrm{N})$ atom sits adjacent to a vacancy $(\mathrm{V})$ along the [111] direction, resulting in a $\mathrm{C}_{3 V}$ point group symmetry. Reprinted with permission from Macmillan Publishers Ltd: Nature Communications [46], copyright 2012. (Author permission pending). (c) Photoluminescence spectrum of $\mathrm{NV}^{-}$at $300 \mathrm{~K}$ and $77 \mathrm{~K}$. A reduction in dephasing results in a narrowed zero-phonon line at cryogenic temperatures, whilst the phonon side-band profile remains dominated by rapid transitions through the vibrational sublevels.

levels provides an asymmetric relaxation pathway (figure 3a) that allows for the non-resonant optical initialisation and readout of the spin state. Since its identification at the single defect level [34, 35], the dual demonstrations of coherent control of the ground state electron spin [36], along with implementations of basic two-qubit gates through coupling with local nuclear spins $\left({ }^{13} \mathrm{C}, \mathrm{I}=\frac{1}{2}\right)[37,38]$, represented the emergence of $\mathrm{NV}^{-}$as one of the pre-eminent solid-state qubit candidates. The phenomenal progress made over the last decade has seen the establishment of the underlying physics [39], development of coherent electron and nuclear spin control techniques and improvements in the process control of both diamond crystal growth and defect engineering. The electron spin coherence can be extended through isotopic purification during diamond growth (to suppress the $1.1 \%$ natural ${ }^{13} \mathrm{C}$ abundance)[5]. The application of dynamical decoupling protocols $[40,41,42]$, which serve to reverse phase accumulated by the interaction with the ${ }^{13} \mathrm{C}$ nuclear spin-bath, can further extend the coherence. Mediated by the hyperfine interaction, the electron has access to well-isolated long-lived nuclear spin states (using ${ }^{13} \mathrm{C},{ }^{14} \mathrm{~N}$ $(\mathrm{I}=1)$ or $\left.{ }^{15} \mathrm{~N}\left(\mathrm{I}=\frac{1}{2}\right)\right)$ [7] and extended coherence has enabled rapid nuclear spin-initialisation [43] and projective single-shot readout $[44,45]$. Recent developments have seen the implementation of quantum error correction protocols on these multi-qubit registers, consisting of a single electron spin and up to three nuclear spins [26, 27], underlining the rich computational resources that are available to defects in diamond.

\section{$N V^{-}$Optical Transitions}

Relaxation from the ${ }^{3} E$ excited state is dominated by radiative transitions to the ${ }^{3} A_{2}$ ground state, 

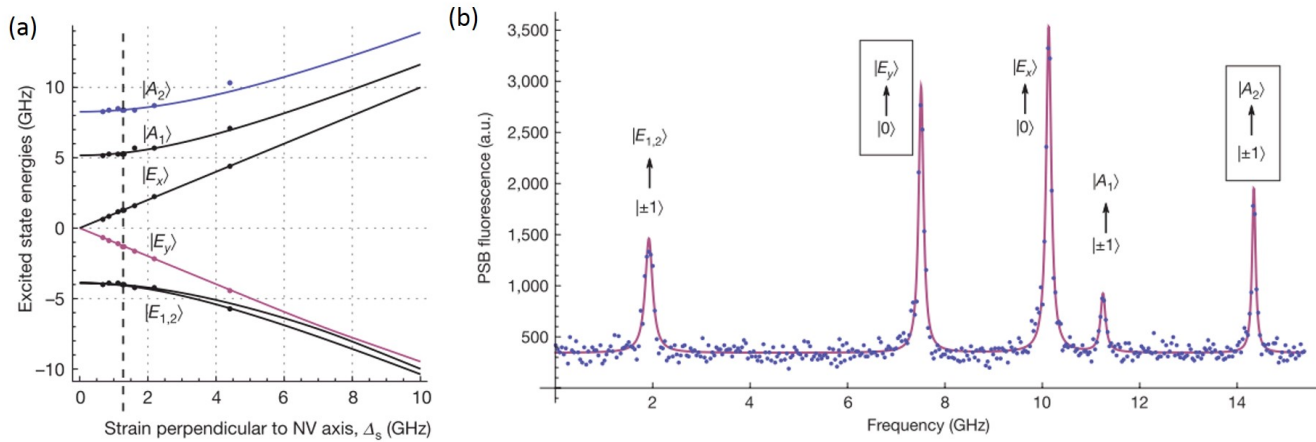

Figure 4: (a) The fine structure of the NV excited state resolved at cryogenic temperatures as a function of transverse strain. (b) PLE spectrum of the excited state. It is necessary to apply a modulation of $2.88 \mathrm{GHz}$ to the resonant excitation laser such that the transitions from the $| \pm 1\rangle$ projections can be observed. Reprinted by permission from Macmillan Publishers Ltd: Nature [31], copyright 2010

which in bulk material collectively occur at a rate of $\gamma=80 \mathrm{MHz}$ giving the defect an excited state lifetime of $13 \mathrm{~ns}$. The excited state fine structure is resolved at cryogenic temperatures around 4K through high-resolution photoluminescence excitation (PLE) spectroscopy as shown in figure 4(b) $[47,31]$. Each state is labelled according to the symmetry of the orbital and its spin projection. The excited state energies are affected by local lattice strain as shown in figure 4(a). Temperatures below 10K render thermally-induced Jahn-Teller dephasing negligible $[48,49]$ and the resulting transition line widths are transform limited [50], fulfilling one of the primary conditions for generation of indistinguishable photons.

For implementation of distributed entanglement protocols, the spin-dependent ${ }^{3} A_{2} \leftrightarrow{ }^{3} E$ electric dipole transitions provide a few different options. For the initialisation of the electron spin into the $\mathrm{m}_{s}=0$ state, resonant excitation of transitions with the $\mathrm{m}_{s}= \pm 1$ spin-projection can achieve a much higher fidelity (preparation error $0.3 \%$ ) than when using non-resonant $532 \mathrm{~nm}$ pumping (preparation error 11\%) [9]. If entanglement between the electron spin and the polarisation state of a photon is desired, the $A_{1}$ excited state can be utilised as demonstrated by Togan et al [31], exploiting the fact that it couples to the different spins in the ground state manifold via photons of different polarisation. For spin readout in the Barrett \& Kok protocol, a highly spin-preserving transition is desirable and the probability of relaxation via a radiative process should be as close to unity as possible, and the transition between the ${ }^{3} E m_{s}=0$ state and the ${ }^{3} A_{2} m_{s}=0$ state is the most obvious choice.

Despite its many virtues, there are some features of $\mathrm{NV}^{-}$that are less than ideal for photonic networks. Perhaps the most significant of these is that its optical transitions couple strongly to vibrational degrees of freedom resulting in a high proportion of phonon-assisted emission [39] that can not be used for entanglement purposes (although it remains useful for spin readout and monitoring purposes). Just $3 \pm 1 \%[51,52]$ of the emission occurs through the zero-phonon line (ZPL) as shown in figure 3(c), which in the absence of other factors immediately sets an upper limit of $\eta_{e m}<0.03$ in equation 1.

A second limitation is that the low defect symmetry of the NV colour centre renders it highly sensitive to local electric fields and strain. Emitters in the solid-state inevitably experience a 

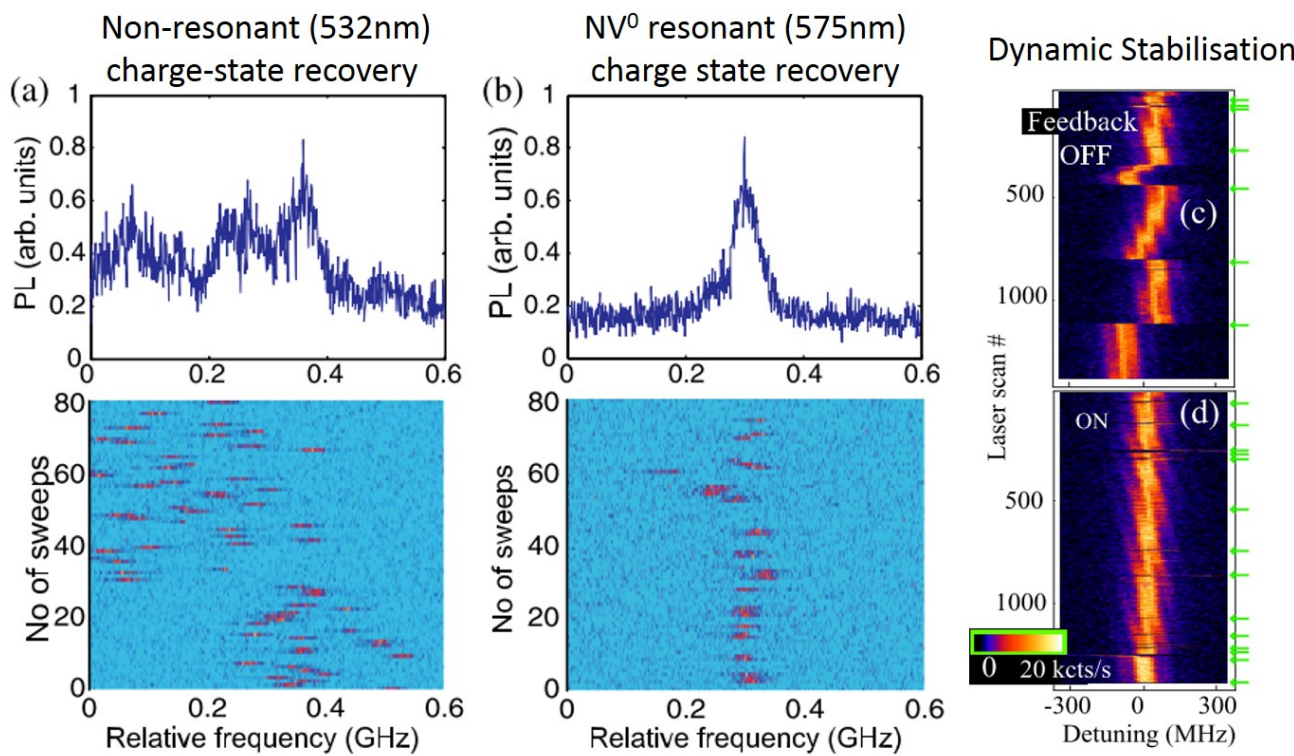

Figure 5: Spectral drift of ZPL transitions in $\mathrm{NV}^{-}$. (a) PLE measurements: a $1 \mathrm{~mW} 532 \mathrm{~nm}$ laser pulse is applied after each resonant laser scan to recover the negative charge state after photoionisation. The averaged spectra are shown on the top panel. (b) A $575 \mathrm{~nm}$ laser resonant with the $\mathrm{NV}^{0}$ transition is applied after every scan. This resonant recovery technique is accomplished with a lower laser power. Reprinted with permission from [55]. Copyright 2013 by the American Physical Society. (c) A PLE trace is shown without any stabilisation applied. The green arrows indicate the points at which a $532 \mathrm{~nm}$ recovery pulse as applied. (d) Using a feedback loop, dynamic stabilisation is achieved via the applied bias voltage of the gate electrodes on the surface. Reprinted with permission from [56].

dynamic electric field environment when under optical excitation, owing to the ionization of surrounding donor and acceptor states. Primary candidates in diamond include substitutional nitrogen (ionized under $532 \mathrm{~nm}$ excitation), vacancies, and $\mathrm{sp}^{2}$ hybridised graphitic carbon [53]. An example of the resulting spectral drift of the electronic transitions can be seen in figure 5a. The effect of such random spectral drift on a networked node is that relative to a fixed wavelength excitation laser, the resonance condition will occur only a fraction of the time, such that the probability of excitation $\eta_{e x}$ will be less than unity. The extent of the spectral drift is characterised by an inhomogeneous line width $\gamma_{\text {inhom }}$, whereby the probability of excitation, given a homogeneous linewidth $\gamma$, is $\eta_{e x}=\frac{\gamma}{\gamma+\gamma_{i n h e}}$. The issue of spectral instability becomes of particular importance for defects that suffer from ionisation, since the desired charge state is typically restored by an optical pulse that is likely to ionise additional defects. Ionisation of the $\mathrm{NV}^{-}$centre to the chargeneutral $\mathrm{NV}^{0}$ state under resonant excitation has been extensively studied [54, 55], along with the resultant spectral drift upon restoration of the negative charge state by non-resonant $532 \mathrm{~nm}$ excitation, which can typically be of order $\gamma_{\text {inhom }} \sim 200 \mathrm{MHz}$ or more (Figure 5a).

Stabilisation of these transitions is therefore of great importance for maintaining resonance with an excitation laser and achieving high $\eta_{e x}$. Resonant excitation of the $\mathrm{NV}^{0} \mathrm{ZPL}$ at $575 \mathrm{~nm}$ has been shown to recover the negative charge state with a much lower excitation power density and consequently reducing $\gamma_{\text {inhom }}$ to $50 \mathrm{MHz}$ or less $[55,57]$, as seen in figure 5(b). As with the ionisation process itself, this resonant repump was proposed to occur via a two photon excitation 
(a)

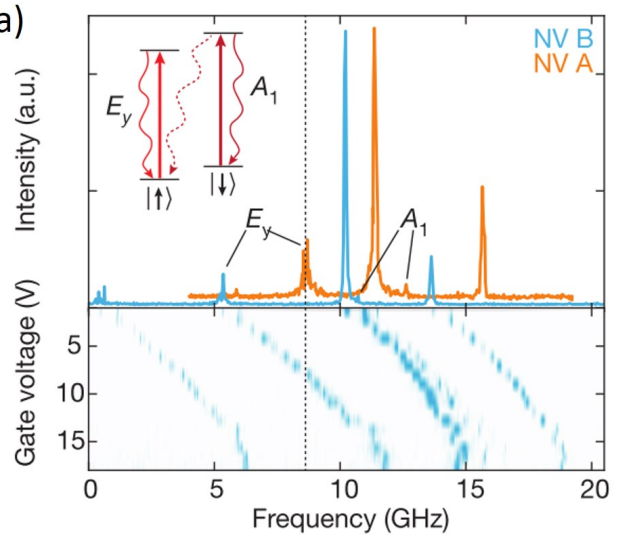

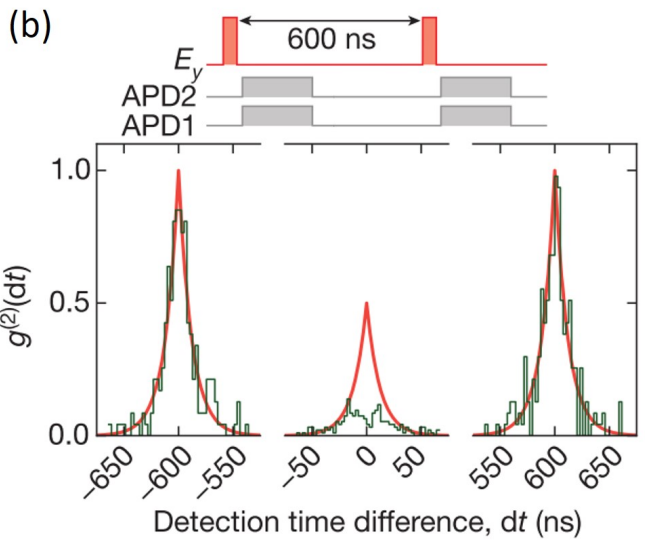

Figure 6: Indistinguishable photon emission from separate NV centres. (a) PLE spectra of each defect showing the relative offset in the transition energies. A gate voltage is applied to NV B enabling the $\mathrm{E}_{y}$ transitions to be tuned into resonance. (b) Above: Excitation pulse and detector gating sequence for the Hong Ou Mandel experiment, Below: photon correlation measurements (green) using pulsed excitation, demonstrating indistinguishable photon emission from each NV with a HOM visibility of $80 \pm 5 \%$. The red curve indicates the expected shape in the case of fully distinguishable emission. Reprinted with permission from Macmillan Publishers Ltd: Nature [11], copyright 2013.

process, followed by diffusion of the deep-lying hole state in the valence band [55]. Acosta et al [56] have also demonstrated dynamic stabilisation of the transition (figure 5(c)), using gate electrodes and a feedback mechanism. A time-integrated line width of $\gamma_{\text {inhom }}+\gamma \simeq 16 \mathrm{MHz}$ was achieved using this method, suggesting that an excitation efficiency of $\eta_{e x} \simeq 0.8$ would be achieved with a single frequency laser. However the usefulness of such dynamic stabilisation techniques is dependent on the speed with which the appropriate correction can be calculated. In the method reported, compensation of both slow drift and larger spectral jumps required scanning of the excitation laser at a maximum rate of up to $20 \mathrm{~Hz}$, taking 5-10 cycles at most to recover the target energy. Most recently a third approach to ZPL stabilisation has been proposed using near-resonant optical pulse trains as a form of optical dynamic decoupling [58] but such a technique has yet to be demonstrated experimentally.

Indistinguishable Photon Emission from $\mathrm{NV}^{-}$

Indistinguishable photon emission from the ZPLs of separate NV centers was first demonstrated by the Delft [59] and Harvard groups [60] via the Hong Ou Mandel (HOM) effect [61]. Both experiments utilised integrated solid immersion lenses (SILs) to enhance photon collection (as discussed in section 4.1), and gate-electrodes for Stark tuning to bring the transition energies of the two defects into resonance with each other (figure 6a) $[50,56]$. The apparatus is essentially the same as that of the Barrett \& Kok entanglement scheme shown in figure 2, with the number of simultaneous detection events on the two detectors monitored as the two nodes are repeatedly excited. If the photons emitted from the nodes are distinguishable then the central peak of the coincidence histogram (figure 6(b)) at $d t=0$ will be no less than half the height of the peaks at $d t= \pm 600 \mathrm{~ns}$. A central peak height below this value indicates two-photon quantum interference effects, and perfect indistinguishability results in the complete absence of the central peak. The results demonstrate an indistinguishability of $80 \pm 5 \%$. 


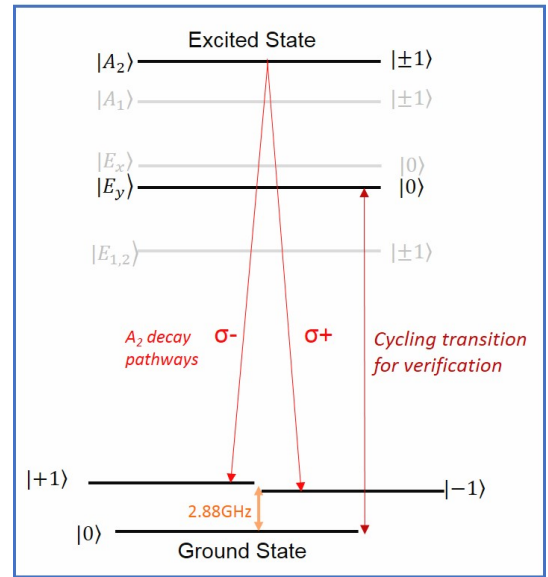

Figure 7: Scheme for NV spin-photon entanglement. A $\Lambda$ transition connects $|+1\rangle$ and $|-1\rangle$ to a common excited state $\left|A_{2}\right\rangle$. The cycling transtion $\left|E_{y}\right\rangle$ is used for read-out to confirm correlations between the measured photon-polarisation and the spin population. Adapted from [31].

\section{Spin-Photon Entanglement with $\mathrm{NV}^{-}$}

The demonstration by Togan et al [31] of spin-photon entanglement with $\mathrm{NV}^{-}$relies on the fact that the excited level $\left|A_{2}\right\rangle$ is an entangled state of spin $\left|m_{s}= \pm 1\right\rangle$ and orbital $\left|E_{ \pm}\right\rangle$angular momentum projections. This decays with equal probability to the ground states $\left|m_{s}= \pm 1\right\rangle$ through the emission of circularly polarised photons of opposite helicity, as shown in figure 7. The resulting state after the relaxation process is complete is the entangled state $|\psi\rangle=$ $\frac{1}{\sqrt{2}}\left(\left|\sigma_{-}\right\rangle\left|m_{s}=+1\right\rangle+\left|\sigma_{+}\right\rangle\left|m_{s}=-1\right\rangle\right)$ and measurements in the circularly polarised $\left|\sigma_{ \pm}\right\rangle$basis verified the emitted photons to be entangled with the spin states $\left|m_{s}= \pm 1\right\rangle$.

Spin-photon entanglement of this kind can be used to generate distributed entanglement between two nodes heralded by a single coincidence event [62], but is not well suited to the growth of scalable networks since the quantum state of the node is reset to the common excited state during each step. The Barrett \& Kok scheme instead utilises selective excitation to entangle the spin state with the presence of a photon, with the benefit that any existing entanglement with other nodes is preserved following a successful entanglement operation.

\section{Remote Spin-Spin Entanglement with $\mathrm{NV}^{-}$}

The seminal work by the Delft group to entangle two NV spins [11] utilised the Barrett \& Kok proposal [15] introduced in section 1.1 with the relevant steps outlined schematically in figure 8 . Here the $m_{s}=0$ and $m_{s}=-1$ states are denoted as $|\uparrow\rangle$ and $|\downarrow\rangle$ respectively. Both NVs were first prepared into the coherent superposition state $\frac{1}{\sqrt{2}}\left(|\uparrow\rangle+|\downarrow\rangle\right.$ by pumping the $|\downarrow\rangle \leftrightarrow\left|A_{1}\right\rangle$ transition to produce $|\uparrow\rangle[9]$ and then applying a $\frac{\pi}{2}$ microwave pulse to create the spin superpositions. An excitation pulse resonant with the $\left|E_{y}\right\rangle$ transition was then applied to each $\mathrm{NV}$, resulting in local entanglement of the spin with the photon number (emission $=1$, no emission $=0$ ) at each NV site creating the state $\frac{1}{\sqrt{2}}|\uparrow 1\rangle+|\downarrow 0\rangle$. The emission channels are directed onto a 50:50 beamsplitter and two-photon interference provides the necessary path erasure. The successful detection of a photon in each of the two phases of the double heralding scheme projects the two NV spins 


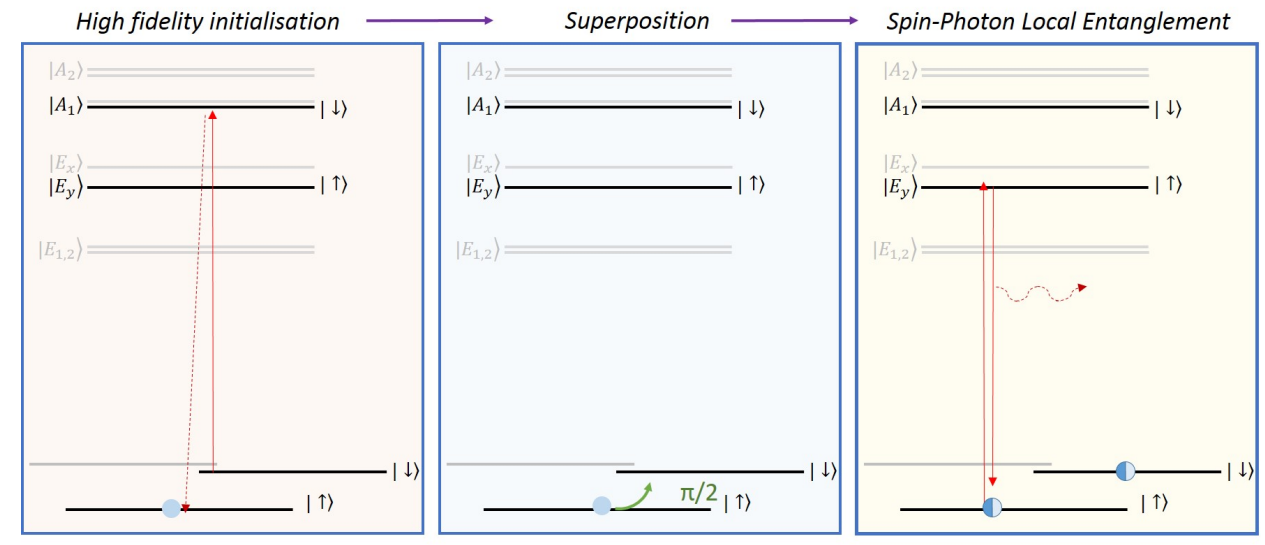

Figure 8: Generation of remote spin-spin entanglement through the Barrett \& Kok protocol applied to the NV centre The relevant ground and excited state levels are indicated in black. (a) Each system is initialised into $|\uparrow\rangle$. (b) A $\pi / 2$ microwave pulse prepares a superposition between the $|\uparrow\rangle$ and $|\downarrow\rangle$ states. (c) A resonant pulse is applied to the cycling transition $\left|E_{y}\right\rangle$. Spontaneous emission creates a local entanglement between the spin and the photon number. The output channels from each spin are input onto a 50:50 beamsplitter, described further in text. Adapted from [11].

into the state $|\psi\rangle=\frac{1}{\sqrt{2}}\left|\uparrow_{A} \downarrow_{B}\right\rangle \pm\left|\downarrow_{A} \uparrow_{B}\right\rangle$. The sign of the relative phase between the two terms is determined by whether the two photons were detected by the same detector (in which case it is + , producing the Bell state $\Psi_{+}$) or by opposite detectors (in which case it is -, producing the Bell state $\left.\Psi_{-}\right)$.

The spin-dependent optical measurement is achieved by resonance fluorescence, utilising both optical polarisation and time gating to distinguish fluorescence photons from those scattered towards the detector from elsewhere in the apparatus. The success of this protocol naturally depends on the detection probability $\eta_{i}$, of resonant ZPL photons in each channel, which was found to be $\approx 4 \times 10^{-4}$. This led to an overall sucess rate $P_{\psi} \approx \frac{1}{2} \eta_{A} \eta_{B}=10^{-7}$, where the factor of $\frac{1}{2}$ account for both rounds of the protocol. An attempt frequency of $20 \mathrm{kHz}$ produced an entanglement generation rate of $\sim 1.7 \times 10^{-3} \mathrm{~s}^{-1}$. This rate was increased to $4 \times 10^{-3} \mathrm{~s}^{-1}$ in the subsequent demonstrations of quantum-state teleportation by the same group, upon increasing $\eta_{\text {ex }}$ using the $\mathrm{NV}^{0}$ resonant charge state recovery scheme discussed previously [12]. More recently, this same platform was used to demonstrate loophole-free violations of Bell's inequalities with NVs separated by $1.3 \mathrm{~km}$ [13] setting a new record for distributed entanglement in any system. In this tour-de-force experiment the indistinguishable photons were transmitted from the nodes to the detectors using single mode optical fibres, such that the attenuation of the signal by the fibre of $\sim 8 \mathrm{~dB} / \mathrm{km}$ begins to limit $\eta_{t r}$ and thus the rate of entanglement. The successful implementation of the Barrett \& Kok scheme with a long range fibre network is also significant in that the scheme requires interferometric stability in the entire apparatus during the time elapsed between the two heralding events. That such stability was achieved without any special attention being paid to thermal stabilisation of the fibres is encouraging for further scale-up as efficiencies are improved. 

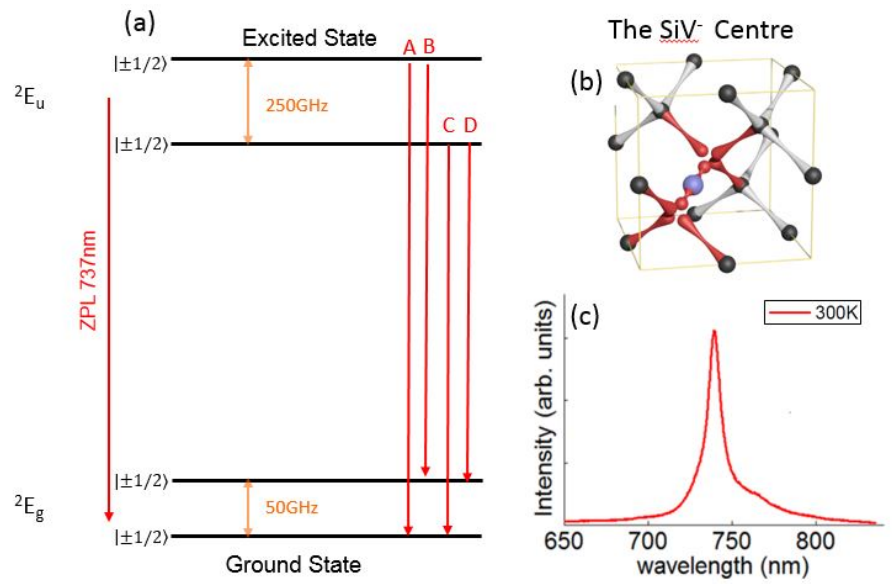

Figure 9: (a) SiV energy level structure, with the ZPL transition at $737 \mathrm{~nm}$ indicated. A zero-field splitting (orange) exists within the ground and excited state doublets giving rise to four transitions which can be resolved at low temperature. Adapted from [63]. (b) Crystal Structure: a silicon atom (purple) sits at an interstitial position between two vacancy sites (dangling bonds in red), along the [111] direction. Reprinted from [74]. (c) Room temperature spectrum of the SiV. Most of the emission originates from the ZPL transition at $737 \mathrm{~nm}$. Reprinted with permission from [75]. Copyright 2015 American Chemical Society.

\subsection{The Silicon-Vacancy Centre}

The SiV centre, illustrated in figure 9, possesses optical properties that are in some important respects superior to those of the NV centre, and recent demonstrations of optically accessible spin-sublevels in SiV [63] have suggested its potential for use as a networked quantum node. The physical structure of $\mathrm{SiV}$, shown in figure $9 \mathrm{~b}$, comprises a silicon atom in an interstitial site within a split vacancy along the [111] axis [64, 65]. As with NV it is the negatively charged defect $\left(\mathrm{SiV}^{-}\right.$, with $\mathrm{ZPL}$ at $\left.738 \mathrm{~nm}\right)$ that is of greatest interest. In contrast with $\mathrm{NV}$ however the $\mathrm{SiV}$ possesses inversion symmetry, giving rise to quite different optical properties. In particular the electronic transitions interact only very weakly with lattice distortions allowing $70 \%$ of emission into the ZPL [66] as can be seen in figure 9(c). Line widths of individual transitions are as narrow as $0.7 \mathrm{~nm}$ at room temperature [67], and photon count rates of up to 6.2 MCps have been observed from single $\mathrm{SiV}^{-}$centres in CVD nanodiamond grown on iridium [68]. With strong narrow $\mathrm{ZPL}$ transitions, the $\mathrm{SiV}^{-}$is well suited for integration into resonant photonic structures, with significant cavity coupling effects observed even at room temperature $[69,70,71,72]$. These advantages persist despite a low quantum efficiency, with reports varying from 34\%-66\% in the bulk [71]. Fluorescence lifetimes of single centres in the bulk are around 1-3 ns at ambient temperatures, and about 30\% longer at $4 \mathrm{~K}$, which Rogers et al suggest could be consistent with thermally activated charge traps [66]. The SiV defect is known to have a lower energy transition in the $820-840 \mathrm{~nm}$ region [73], which is responsible for bunching at intermediate time delays in photon correlation measurements $[68,71]$, however the role that this transition plays in the quantum efficiency of the $738 \mathrm{~nm}$ electronic transition remains to be understood.

\section{Spin properties of $\mathrm{SiV}^{-}$}

Eleven electrons are associated with the negatively charged $\mathrm{SiV}$ defect, forming a spin $\frac{1}{2}$ system. The electronic energy level structure of $\mathrm{SiV}^{-}$, shown in figure $9 \mathrm{a}$, has only recently been con- 
(a)

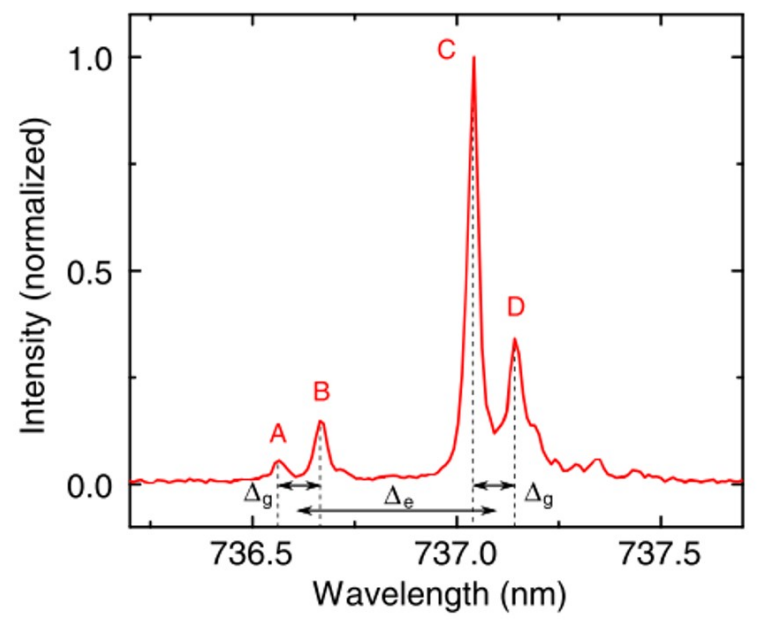

(b)

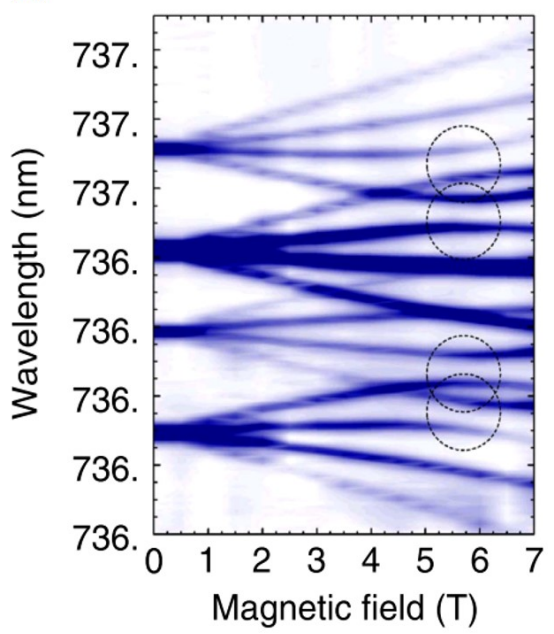

Figure 10: (a) The fine structure of an SiV ensemble at $4 \mathrm{~K}$. There is there is very little inhomogeneous distribution in energy between defects allowing the four transitions between the orbital doublets in the ground and excited state to be resolved. (b) The magnetic field dependence of the ZPL transitions of a single $\mathrm{SiV}$ at 4K, which allows for optical access of the individual spin sub-levels. Reprinted with permission from Macmillan Publishers Ltd: Nature Communications [63], copyright 2014.

firmed $[65,64]$. The ground and excited states are both orbital doublets with spin-orbit splittings of $47 \mathrm{GHz}$ and $258 \mathrm{GHz}$ [76]. These large splittings provide one of the significant attractions of the $\mathrm{SiV}$ centre, that its electronic state can be coherently manipulated on picosecond time scales with optical pulses [77]. At cryogenic temperatures the fine structure is easily resolvable and the four optical transitions at zero-magnetic field are shown in figure 10a. Transitions A \& D are co-polarised, which are orthogonal to the co-polarised inner transitions B \& C. These orientations are in agreement with the [111] alignment of the defect. Zeeman splitting under high magnetic fields lifts the remaining degeneracy of the spin sublevels allowing optical access the spin as shown in figure 10b. Using resonance fluorescence at magnetic fields up to 4T, Muller et al were able to optically access the spin sub-levels in the excited state manifold, with spindependent fluorescence to the ground states identified. By driving optical transitions between the lower branches of the ground and excited state, they were able to demonstrate a contrast of $90 \%$ between fluorescence signal between spin sub-levels [63] indicating high spin-purity. A current drawback of the $\mathrm{SiV}$ for use as a qubit is its relatively short electronic spin coherence time [78, 79], limited to around $100 \mathrm{~ns}$ by phonon-induced scattering between the orbital sublevels in the ${ }^{2} E_{g}$ ground state [66]. Although this coherence time is a reasonably healthy four orders of magnitude longer than the state rotation time, it may be too short to perform high fidelity gates with nearby nuclei as required for for fault tolerance and entanglement purification. Proposals to prolong $T_{1}$, and thereby also $T_{2}$, include cooling to millikelvin temperatures, the use of microstructures to modify the local phonon environment [79], or the application of strain to modify the splitting of the ${ }^{2} E_{g}$ levels. The success of these efforts will be crucial in determining the viability of the $\mathrm{SiV}$ as a spin qubit. 


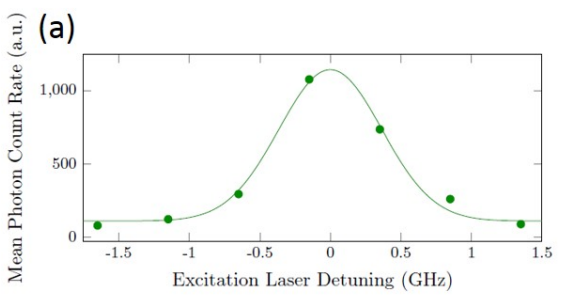

(c)
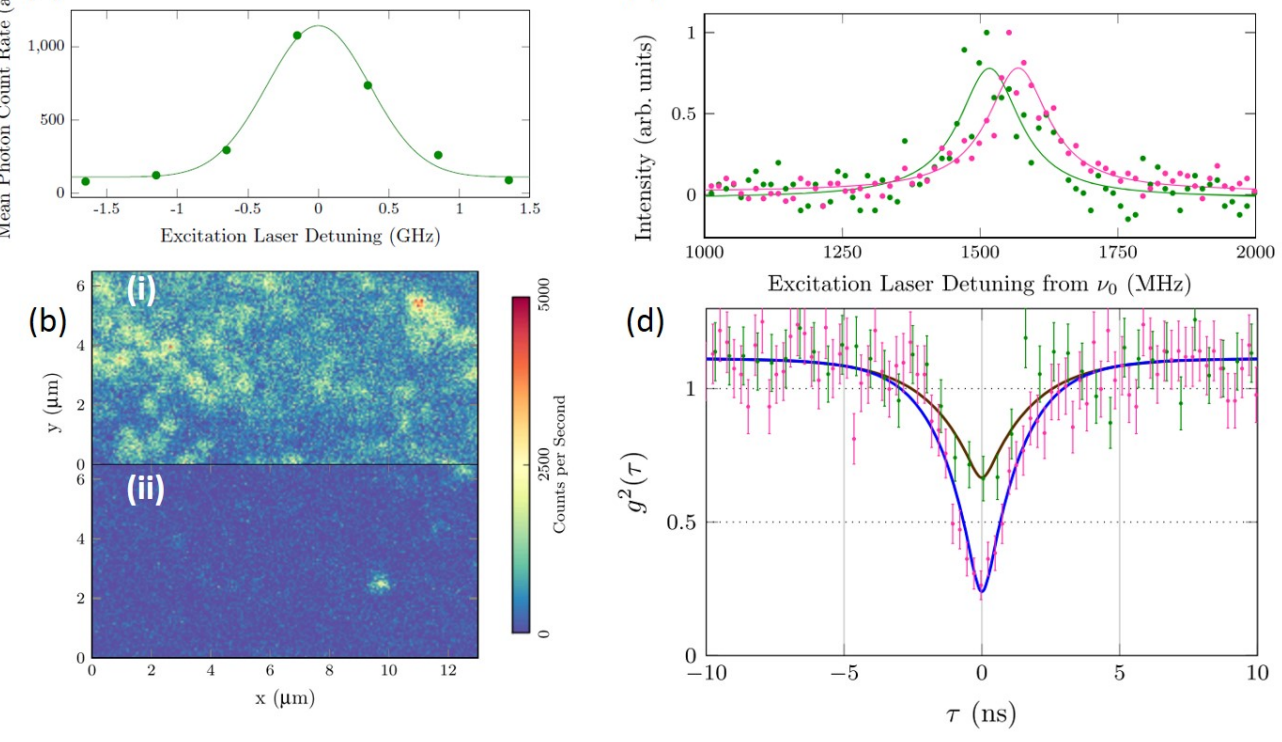

Figure 11: Indistinguishable photon emission from separate $\mathrm{SiV}$ centres in diamond. (a) The inhomogeneous distribution of a single transition for multiple $\mathrm{SiV}$ centres in a $150 \mu \mathrm{m}$ field of view. The total side-band fluorescence from this region is measured as a function of resonant excitation laser frequency resulting in a measured distribution of $365 \mathrm{MHz}$. Reproduced with permission from [80]. (b) PLE image with excitation tuned to (i) the mean of the inhomogeneous distribution and (ii) the edge of the distribution. This way identical SiVs are isolated to reduce the background contribution and selected for HOM experiments. (c) Spectral overlap of the selected SiVs, which have a detuning of less than half of the homogeneous linewidth. (c) Second order correlation functions for orthogonal (green) and co-polarised (pink) emission from distinct SiVs. The co-polarised emission reaches below $\mathrm{g}^{(2)}=0.5$, confirming indistinguishable photon emission. Reproduced with permission from [74]

\section{Coherent Optical Transitions in $\mathrm{SiV}^{-}$}

Owing to the inversion symmetry, the orbital states of the $\mathrm{SiV}$ centre have a vanishingly small permanent electric dipole moment which renders them to be mostly unperturbed by external electric fields [74]. PLE studies of the spectral stability by Rogers et al demonstrated frequency stability to within $8 \mathrm{MHz}$ over 7 hours of study [66] without the need for dynamical stabilisation [56]. Additionally the excited state transitions in high-quality, low strain samples are shown to be spectrally narrow down to $120 \mathrm{MHz}$, nearly at the transform limit corresponding to the fluorescence lifetime of $1.7 \mathrm{~ns}$. The inversion symmetry also ensures a consistency in the spectral positions of the optical transitions between defects. In the work of Rogers et al, over $50 \%$ of the centres studied had transitions within a transform limited lifetime of one another [66]. This was exploited by Sipahigil and coworkers who observed the emission of indistinguishable photons from distinct $\mathrm{SiV}$ centres in the same diamond, demonstrating a HOM-visibility of 0.72 , shown in figure 11a [74]. The uniformity between centres allowed this result to be achieved without the need for Stark shift tuning (figure 11c), as is necessary for NV centres [60, 59]. 


\subsection{Emerging Colour Centres}

Despite a wealth of luminescent defects in diamond [29], only a select few have been identified as single photon emitters [81,30], aside from the NV and SiV. Some of these defects are related to nickel complexes, whilst others remain unidentified despite promising optical properties $[82,83]$.

Of this selection, two have recently shown spin signatures. The ST1 defect was identified after the fabrication of diamond nanowires in single crystal HPHT diamond [83]. The fluorescence spectrum of ST1 shows a ZPL at $550 \mathrm{~nm}$ and a broad PSB. The spin readout contrast is as high as $45 \%$ and the properties are consistent with a spin singlet ground state and intersystem crossing to a triplet manifold. This centre certainly merits further investigation, although relaxation from the metastable triplet states may limit its use as a broker qubit in a quantum network. The germanium vacancy $(\mathrm{GeV})$ has also recently emerged as a potential candidate qubit, with identification of single photon emission [84] and demonstration of coherent spin control [85, 86]. The GeV shares many similiarities with the $\mathrm{SiV}$ centre, as it has the same split-vacancy structure with inversion symmetry along the (111) direction. The relaxation branching ratio from the optically excited state is also favourable for implementation in quantum networks, with approximately $60 \%$ of the photons originating from the ZPL at $602 \mathrm{~nm}$. Furthermore the transitions are largely stable over time [85] and with linewidths approaching the Fourier-transform limit. Of significance are the early signs that the quantum efficiency of the defect could be much higher than that of the $\mathrm{SiV}$, as evidenced by the sensitivity of the transition lifetime on the local optical density of states [86].

\section{Engineering Diamond for Nodes in Quantum Networks}

This section presents the engineering challenges faced in the construction of diamond-based nodes for quantum networks, namely the fabrication of microstructures and position of colour centres within them to maximise the efficiency with which indistinguishable photons can be produced $\left(\eta_{e m}\right)$ and passed from the node to the network $\left(\eta_{c o}\right)$. The most appropriate approach depends to an extent on the colour centre (and protocol) to be used, and progress in realisation of specific devices will be described in section 4 . In this section we first consider the methods available for use in the fabrication process, and some of the recipes that have been developed for achieving key tasks such as fabrication of high quality surfaces and positioning of high quality colour centres within the diamond lattice. In order to produce the required optical properties, these processes must meet stringent demands in terms of the number and type of crystal defects that are generated and the chemistry of nearby surfaces. We consider the methods available for microstructural fabrication of diamond in section 3.1, followed by methods for placing colour centres in photonic structures in 3.2. Section 3.3 concerns the chemical preparation of diamond surfaces, and section 3.4 looks at the best achieved optical properties of colour centres in engineered diamond.

\subsection{Fabrication and Processing}

Diamond grown by chemical vapour deposition (CVD) currently represents the benchmark for the material of high chemical purity required for quantum photonic / spintronic devices. This controlled process allows for impurities to be minimised (section 3.2), or for dopants to be introduced (section 3.2.3), whilst also enabling growth of isotopically pure materials for suppression of spin decoherence [5] (section 2.1). Whilst nanodiamond and polycrystalline materials offer many useful properties, and single colour centres in these materials find uses in single photon 
sources and nano-magnetometry, single crystal material is preferred for applications in which high degrees of both spin coherence and optical coherence are required.

The production of uniform films of single crystal diamond of order $100 \mathrm{~nm}$ in thickness is central to many architectures for single mode photonics (discussed further in section 4.4) but the lack of an established heteroepitaxial growth substrate presents a significant challenge [87, 88, 89]. Instead, membranes have been produced by mechanical polishing [30] and/or laser cutting of bulk materials, prior to being thinned by reactive ion etching (RIE) techniques (3.1.1). Such 'top-down' processes suffer from low reproducibility and tend to result in membranes of nonuniform thickness, with a variation of the order $300 \mathrm{~nm} / \mathrm{mm}[90,91]$, effectively restricting device development to basic proof-of-principle demonstrations. In this respect the commercially grown starting materials may be improved through template-assisted re-polishing [88]. Membranes of uniform thickness have been produced using helium ion bombardment to form a well-controlled sub-surface damaged layer, which is then sacrificed through selective chemical etching followed by lift-out $[92,93,94]$. The residual ion damage in the layer through which the helium ions have passed is deleterious to coherent single colour centre properties [95] (section 3.4), however it has been shown that these membranes can be suitable for the subsequent overgrowth of highquality single crystal material $[96,91]$, which has been combined with the delta-doping method to create high-quality free-standing nanobeam cavities (section 5.1.1) [97]. Coherent optical properties for single colour centres remain to be demonstrated in these structures and a further discussion of these techniques can be found elsewhere [30]. Other fabrication protocols can mitigate the necessity for large uniform membranes, such as the focused ion beam approaches (briefly discussed in section 3.1.2) to undercutting photonic structures [98, 99], angular RIE methods (section 4.4) and the hybrid GaP-on-diamond device designs (section 5.1.3). Diamond nanofabrication is an extensive subject area and further discussions can be found in references $[100,30,101,89]$.

\subsubsection{Reactive Ion Etching}

Reactive ion etching is a widespread and versatile technique and has become indispensable for top-down semiconductor fabrication processing. Under an applied bias, plasma ions are accelerated towards the surface of the material, breaking down and removing material through a combination of ion bombardment and chemical reaction. For applications in diamond, both of these roles may be fulfilled by one species, as in pure $\mathrm{O}_{2}$ based plasma recipes $[102,51,103$, 104]. Alternatively these roles may be separated using mixtures of $\mathrm{Ar} / \mathrm{O}_{2}$ or $\mathrm{Ar} / \mathrm{Cl}_{2}[105,106$, 107, 108] which can offer a more controlled process [101]. Each of these methods has their relative merits and drawbacks with regards to the surface termination, surface roughness and sub-surface lattice damage.

Inductively coupled plasma (ICP)-RIE recipes using $\mathrm{Ar} / \mathrm{Cl}_{2}$ have been shown to produce the smoothest surfaces, with Lee et al demonstrating a reduction in roughness from $\sigma_{\mathrm{RMS}}=0.53$ to $0.19 \mathrm{~nm}$ over a 10 minute etch cycle [108]. The $\mathrm{Ar} / \mathrm{Cl}_{2}$ etch does not however provide a 'one-stop shop' solution to the etching of diamond microstructures for quantum networks, as the process has been widely reported to quench near-surface NV fluorescence $[109,30,110]$. Part of the explanation for this effect comes from X-ray photoelectron spectroscopy of the postprocessed surface showing chlorine signatures [111, 110], and theoretical calculations showing that chlorine cannot fully terminate a diamond surface leading to a mixed passivation environment unfavourable for stable NV emission [112]. However these results do not appear to explain the near-complete suppression of NV emission that occurs within a few micrometres of an $\mathrm{Ar} / \mathrm{Cl}_{2}$ etched surface, and a full explanation is still lacking. State of the art fabrication recipes 
therefore utilise $\mathrm{O}_{2}$ based plasmas to remove the last few micrometres of material, which allows the retention of smooth surfaces whilst removing the deleterious effects of the $\mathrm{Ar} / \mathrm{Cl}_{2}$ etch [109, 57, 113, 114, 115, 52].

Etching conditions such as applied bias power and even the type of reaction chamber are important for the minimisation of sub-surface lattice damage. Studies have shown that both pure $\mathrm{O}_{2}$ plasma [116] and $\mathrm{Ar} / \mathrm{O}_{2}$ mixtures [117] may damage the surface at high bias powers, resulting in the quenching of near-surface NV fluorescence $[116,118]$. This damage can be mitigated by the use of 'soft' oxygen plasma recipes [119]. Cui et al demonstrated the extent of sub-surface damage with oxygen etching to be dependent on the type of reactor used, comparing a standard ICP-RIE system with plasma barrel reactors (lowered bias) and downstream etchers (sample location and plasma generation separated) as more gentle alternatives [118]. Using delta-doping (see section 3.2.3) to position NVs $70 \mathrm{~nm}$ below the initial sample surface, NV fluorescence was shown to switch off after just $30 \mathrm{~nm}$ of etching in the ICP-RIE system. As expected, downstream etching is observed to be most gentle, with centres still present after $55 \mathrm{~nm}$ of etching. Though these exceed the requirement for colour-centre-surface proximity in photonic structures $(\approx 60$ $75 \mathrm{~nm}$ ), they serve to demonstrate the sensitivity of the emitter properties to the subtleties of the chosen processing method.

\subsubsection{FIB Milling}

Focused-ion beam (FIB) milling has found application in the nanostructuring of diamond to create photonic structures such as SILs $[120,121,122]$ and photonic crystals $[123,98,124,70$, $71,99]$. FIB processing sputters the target material with heavy-ions, such as gallium, at high acceleration voltages, and as such creates significant damage to the surface, including graphitisation [70] and gallium ion implantation [123]. Such damage is acceptable for structures such as the SIL in which the colour centre can be several micrometres from the surface, but presents a challenge to nanophotonic structures such as photonic crystals where the colour centre is within $100 \mathrm{~nm}$ of the surface. In the latter case post-processing methods are required - annealing at a temperature over $1000 \mathrm{C}$ causes implanted $\mathrm{Ga}^{+}$ions and vacancies to migrate to the diamond surface, with subsequent thermal etching (heating to $~ 450 \mathrm{C}$ in an oxygen-rich atmosphere) and acid boiling enabling the removal of surface damage and contamination [70].

\subsection{Generation and Placement of Defects}

As is the case for a large number of diamond colour centres [29], the NV and SiV are complexes of one or more impurities and vacancies. For CVD-grown diamond the impurity atoms may be either introduced during growth or implanted later. Nitrogen is readily incorporated into the diamond lattice during growth owing to its relatively similar size to carbon, and as such it persists even in the most pure commercially available type IIa materials, in quantities down to a few parts per billion [101, 125, 126]. Whilst nitrogen impurities will most commonly take the simple substitutional form, some NVs can also form during the CVD process [126]. These 'native' NVs are generally randomly oriented in (100)-grown diamond, but preferential orientation along the growth axis during (111)-oriented growth has been reported, with a probability of over $95 \%$ [127, 128]. Such orientation may offer benefits to photonic devices in which the optical mode is polarised in the (111) plane [129]. Intentional nitrogen doping during growth can be also be achieved through the selective introduction of $\mathrm{N}_{2}$ gas [130] (see section 3.2.3). For $\mathrm{SiVs}$, formation during growth can occur through either silicon contamination from chamber side-walls (containing $\mathrm{SiO}_{2}$ ) $[64,66,131]$, through selection of a silicon/silicon-based growth 
(a)

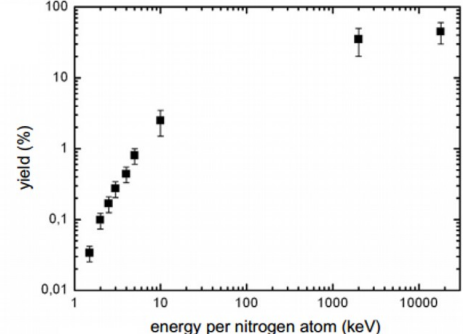

(b)

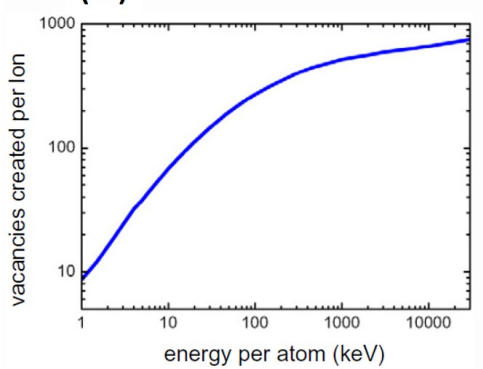

(c)

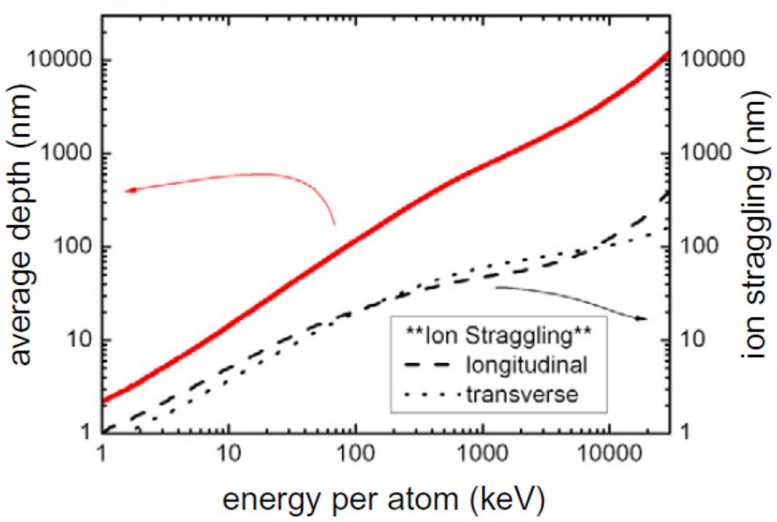

Figure 12: Nitrogen ion implantation in diamond. (a) The NV creation yield is measured as a function of implantation energy, deduced from the total fluorescence intensity compared to the integrated ion fluence. (b) SRIM simulations of the number of vacancies created as a function of implantation energy. (c) SRIM simulation of average implantation depth as a function of energy (red). The longitudinal and transverse straggle is also shown (dashed). Reprinted with permission from [134].

substrate $[64,66,75,81]$ or from the introduction of silane to the precursor gases $[132,133]$.

For the realisation of large scale quantum networks using diamond it is highly desirable to be able to position colour centres within photonic structures with a high production yield. The spatial precision required depends on the architecture adopted. For example, for a single mode resonator such as a photonic crystal cavity (PCC) operating in the visible/NIR region of the spectrum (see section 5.1.1) this length scale is given by $\frac{\lambda}{4 n} \sim 60-75 \mathrm{~nm}$. In some hybrid approaches to resonant enhancement such as open cavities (section 5.2), the requirements are relaxed by the ability to align the resonator and colour centre in-situ.

Since the colour centres of interest are formed by the binding of impurities and vacancies, positioning of colour centres at desired locations is achieved by positioning either or both species within the lattice prior to annealing. The methods available for doing so all involve some degree of damage to the diamond lattice which must then removed as far as is possible to produce colour centres with the required coherent properties. This can be achieved by annealing at higher temperatures as discussed in section 3.4.

\subsubsection{Ion Implantation}

Ion implantation has become the most widespread technique for generating shallow NV [135, 136, 137, 125, 57] and SiV [138, 139, 64, 78, 140, 141] colour centres, and has been the subject of previous reviews $[126,142]$. The impurity is implanted within a few micrometres of the diamond 
surface, with vacancies (and other lattice damage) generated as the ion transfers its kinetic energy to the diamond lattice and comes to rest. The vacancies are mobilised by subsequent annealing at temperatures above $600 \mathrm{C}[142,143]$, and a small percentage of these bind to implanted impurity atoms to form the desired colour centres.

Figure 12a shows data recorded by Pezzagna and co-workers for NV generation via nitrogen ion implantation [134], reporting the NV conversion yield after annealing, as a function of implantation energy, along with a simulation of the number of vacancies generated in figure $12 \mathrm{~b}$. For NV, the conversion yield has been shown to be highest at annealing temperatures of around $800 \mathrm{C}[144,57]$. Increasing the conversion yield continues to be the subject of considerable effort. One of the limitations of the single step implantation process is that the vacancies created are mostly at a shallower depth than the final location of the implanted impurity. To this end, carbon co-implantation has been used to generate vacancies closer to the nitrogen to increase the conversion yield [145]. Vacancy generation using other lightweight ions such as helium may help to minimise residual lattice damage [146, 147].

It is common to implant the nitrogen-15 isotope, in order to differentiate from the native impurities [143]. Figure 12c shows a SRIM (Stopping Range of Ions in Matter) simulation [148] demonstrating the dependence on the average depth of the implanted ions, with the acceleration energy [134]. As the implantation depth increases so too does the ion straggle, as the ions scatter at the lattice sites, which begins to limit the placement precision. For depths around $100 \mathrm{~nm}$ or less, this deviation is below a few tens of nanometres [134, 149], within reasonable tolerances for placement into single mode photonic structures. Implanted nitrogen ions are capable of producing NVs with spin coherence almost on a par with native defects, with $\mathrm{T}_{2} \approx 350 \mu$ s [150], although coherence times of $100 \mu$ s or less are commonly reported [151, 114, 109].

There are several approaches to the positioning of implanted ions. Focused ion beams provide a mask-less approach which can be used to determine the nitrogen implantation region [135], with the lateral precision defined by the beam FWHM down to $100 \mathrm{~nm}$ or less at the focus [152]. For deep fabrication at MeV acceleration energies, the single NV limit has been reached [135], however single NV generation has not been widely reported for shallow $(\approx 20 \mathrm{~nm})$, low energy implantation [152]. For the $\mathrm{SiV}$, focused $60 \mathrm{keV}$ silicon ion implantation has been shown by Tamura and coworkers to reach the single centre limit, implanting $\approx 40 \mathrm{~nm}$ below the surface [139]. Restricting the focused ion beam using a nano-aperture in an AFM tip can further increase the positioning accuracy to $\sim 25 \mathrm{~nm}$ [153], and Riedrich-Möller et al have taken this method further to demonstrate precise placement of NV defects at the centre of photonic crystal cavities (discussed in section 4) [72]. Further work to lower the ion fluences will enable the single NV limit to be reached.

A more prevalent method to achieve nanoscale positioning has been to use shadow masks containing nano-apertures through which ion implantation can be achieved over a larger sample area. Strategies include patterning apertures down to $30 \mathrm{~nm}$ in size via electron beam (e-beam) lithography in resist layers of 200-300 nm [156, 157, 154], fabricating arrays of single emitters as shown in figure 13(a,b). Toyli and coworkers formed on average $1.4 \mathrm{NV}$ centres per aperture upon 10-30 keV implantation through e-beam resist (ZEP520A), achieving a 5\% ion-NV conversion yield [156]. $\mathrm{T}_{2}$ measurements were presented but limited to $23 \mu \mathrm{s}$, which was attributed mainly to charge-state switching, due to surface proximity, and the fact that $\approx 30$ paramagnetic ions ${ }^{15} \mathrm{~N}$ are implanted per aperture. Single defect generation has also been achieved with aper- 


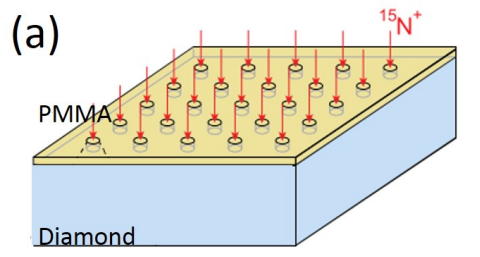

(c)

(b)
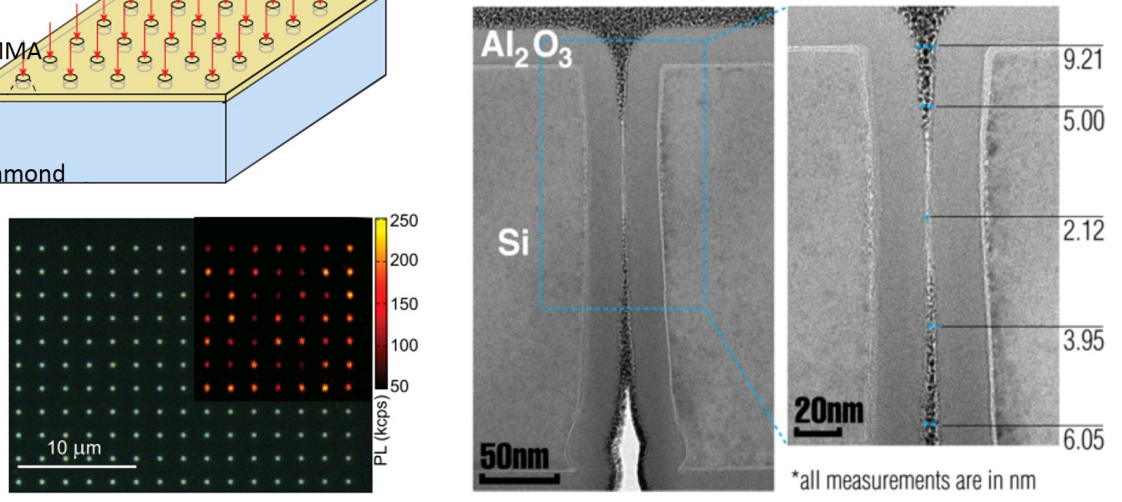

*all measurements are in nm

Figure 13: Precise positioning of colour centres using ion implantation.(a) Electron beam lithography is used to pattern nanoholes in a $230 \mathrm{~nm}$ PMMA layer on the diamond surface, which masks the ion implantation. A dark-field optical microscope image of the mask can be seen in (b), overlaid with the NV fluorescence post-annealing. Reprinted from [154], with permission from AIP Publishing. (c) Cross sectional SEM of a silicon hard-mask with a $47 \mathrm{~nm}$ starting aperture. Atomic layer deposition of $\mathrm{Al}_{2} \mathrm{O}_{3}$ creates a channel of width $2 \mathrm{~nm}$. Reprinted with permission from [155]. Copyright 2015 American Chemical Society.

tures in PMMA, with Spinicelli et al showing a 7\% conversion efficiency and $3.5 \mathrm{NV} /$ aperture through $\mathrm{CN}^{-}$molecular ion beam implantation [157], and Sangtawesin et al demonstrating 6$7 \%$ yield with 0.9 to $5 \mathrm{NV} /$ aperture, dependent on the aperture size [154]. Recently Scarabelli and coworkers gold-coated the diamond surface prior to PMMA coating for e-beam lithography, showing that with post-processing, nano-apertures as small as $15 \mathrm{~nm}$ could be fabricated for masking ion implantation and single NV generation [158]. To achieve smaller apertures with ebeam lithography requires thinner resist layers, however one eventually encounters the problem of implantation through the resist, outside of the defined aperture [155]. High aspect ratio hardmasks have been explored to to circumvent this issue $[159,155]$. Nanochannels down to $20 \mathrm{~nm}$ in width have been demonstrated in mica foils of thickness 5-30 $\mu \mathrm{m}$, fabricated through heavy ion bombardment and subsequent etching [159]. This method was used to mask high energy (1 $\mathrm{MeV})$ nitrogen implantation and fabricate deep $(730 \mathrm{~nm}) \mathrm{NV}$ centres for demonstrations of room temperature NV-NV entanglement through mutual dipolar interaction [160]. Silicon hard-masks are a particularly promising method for combining a high degree of mask isolation with high spatial precision. After fabricating channels of diameter as small as $10 \mathrm{~nm}$ in silicon by e-beam lithography and dry-etching, Bayn and coworkers used atomic layer deposition of $\mathrm{Al}_{2} \mathrm{O}_{3}$ to narrow the channels still further [155], as illustrated in figure 13(c), achieving a minimum channel diameter approaching $2 \mathrm{~nm}$. The fabricated NV distribution, measured by super-resolution imaging was as little as $26 \mathrm{~nm}$ only limited by straggle (6-20 keV energy regime), and this technique has recently been integrated into the fabrication of photonic crystal nanobeam structures [149]. The authors reported a $26 \%$ single NV yield at depths of $110 \mathrm{~nm}$ with $20 \mathrm{~nm}$ of straggle expected.

\subsubsection{Vacancy Generation}

With sufficient concentration of pre-existing impurity atoms, colour centres such as NVs may be fabricated in diamond through the introduction of crystal vacancies alone. It was established 


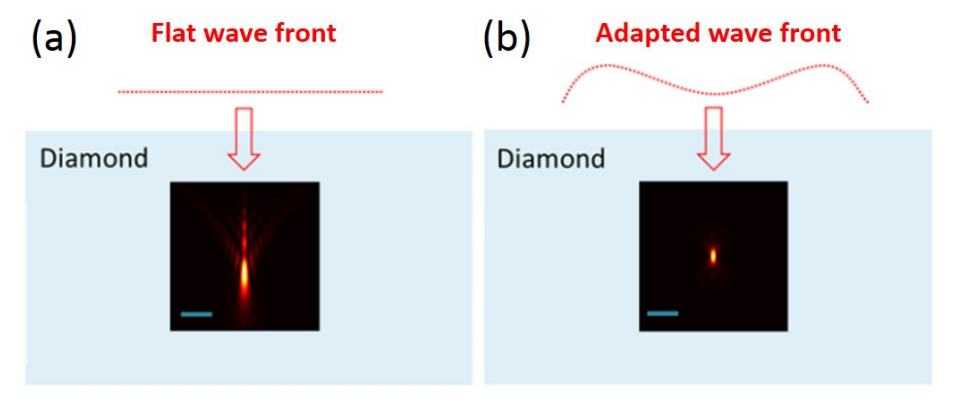

Figure 14: Principle of aberration-free laser writing of vacancies using a femtosecond pulse of a Ti:sapphire laser. (a) A flat wavefront leads to spherical aberrations of the point spread function leading to non-localised damage along the optical axis. A simulation of the point-spread function is shown inset, the scale bar is $2 \mu \mathrm{m}$. (b) An adapted wavefront will localise the laser writing process. Adapted from [165].

in some of the earliest studies on optically active defects in diamond that vacancy generation could be achieved through electron beam irradiation of the lattice, shown to activate NV centres in nitrogen rich type Ib materials upon annealing [161, 34]. Focused electron beam irradiation allows localisation of vacancy generation in two dimensions to within $\sim 10 \mathrm{~nm}[162,163]$, but provides no depth positioning. A different approach has recently been taken by Chen et al, using single pulses from a femtosecond Ti:Sapphire laser to write vacancies into the lattice [164]. The highly nonlinear process of vacancy generation using ultrafast optical pulses means that vacancies are generated only at the most intense region of the laser focus, providing both depth positioning and spatial resolution below the optical diffraction limit. In Chen's work, vacancies were introduced $50 \mu \mathrm{m}$ below the surface and single NV centres were formed with native nitrogen impurities upon annealing. By using adaptive optics to correct for aberrations and minimise the point spread function $[165,166]$ (shown in figure 14), the NVs were positioned to within about $200 \mathrm{~nm}$ in the image plane and $700 \mathrm{~nm}$ in depth. Importantly, some of the NVs were shown to be of high quality, with coherent ZPL transitions at $4 \mathrm{~K}$.

\subsubsection{Delta-Doping for Colour Centre Positioning}

Delta-doping has emerged as a technique to confine impurity doping to a well defined depth, so that only transverse positioning of vacancies is needed to generate accurately placed colour centres. For NV production using this method, $\mathrm{N}_{2}$ gas is briefly introduced to the CVD chamber [130] during growth, creating a nitrogen-rich layer as thin as $2 \mathrm{~nm}$ [167]. Controlled overgrowth then defines the final depth of this layer. Ohno and coworkers used defocused electron irradiation to yield $\mathrm{NV}$ centres confined to within a $8 \mathrm{~nm}$ layer after annealing. Long spin coherence times were also achieved by switching between ${ }^{13} \mathrm{CH}_{4}$ and isotopically pure ${ }^{12} \mathrm{CH}_{4}$ source gases to create a nuclear spin-free buffer around the delta-doped layer as in figure 15, with recorded values from $T_{2}>100 \mu$ s for NVs $5 \mathrm{~nm}$ below the surface to $T_{2}>600 \mu$ s for NVs deeper than $50 \mathrm{~nm}$. Subsequent work demonstrated lateral positioning of vacancy generation using ${ }^{12} \mathrm{C}$ ion bombardment through nano-apertures down to $50 \mathrm{~nm}$ in PMMA [168]. Here the delta-doped layer was $\approx 50 \mathrm{~nm}$ below the surface, whilst the sub-surface vacancies were created at a more shallow depth. During annealing the vacancies diffused to the doped layer, allowing the NVs to form away from the damaged region, manifesting in coherence times $T_{2}>300 \mu \mathrm{s}$. The formation confined the $\mathrm{NVs}$ to a $(180 \mathrm{~nm})^{3}$ volume with on average $\approx 0.9 \mathrm{NVs}$ /aperture. More 


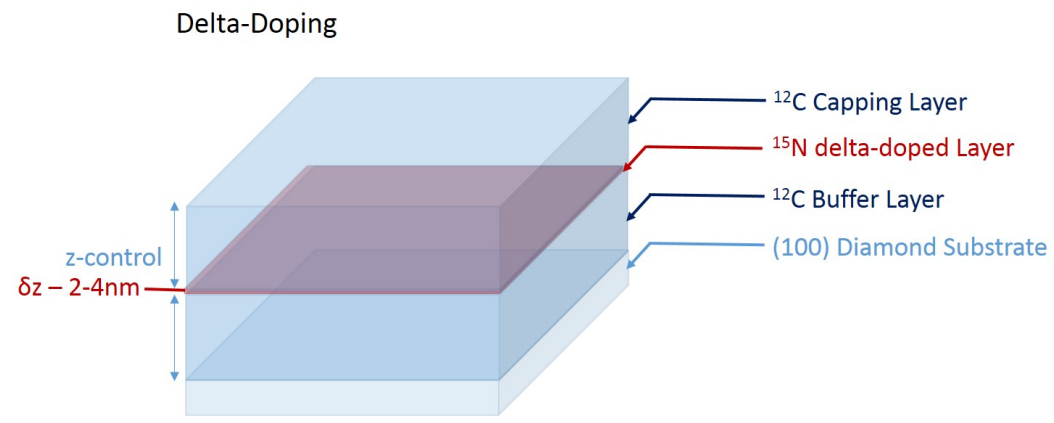

Figure 15: The delta-doping process. $\mathrm{A}^{12} \mathrm{C}$ layer is grown on a (100) diamond substrate. A controlled introduction of ${ }^{15} \mathrm{~N}$ during the growth process allows for a precise nitrogen doped layer. After more overgrowth, vacancies are generated before annealing to form NVs. Adapted from [167].

recent work by McLellan et al used focused electron beam irradiation with spot sizes down to $20 \mathrm{~nm}$ to confine the vacancy generation in a delta-doped sample [169]. This method appears capable of positioning NVs with $<10 \mathrm{~nm}$ accuracy in all three dimensions.

\subsection{Diamond Surface Preparation}

Controlling the surface chemistry is critical to maintaining the $\mathrm{NV}^{-}$charge state and in retaining good optical and spin coherence of the colour centres. Oxygen termination of the surface has been used extensively to achieve good properties, while hydrogen termination, the state of the surface immediately after the CVD growth process [101], forms a conductive surface layer that ionises sub-surface $\mathrm{NV}^{-}$to $\mathrm{NV}^{0}[170,171]$. Fu et al compared the influence of hydrogen and oxygen terminated surfaces on the $\mathrm{NV}^{-} / \mathrm{NV}^{0}$ ratio, at implantation depths of 14-62 nm [53]. With $\mathrm{NV}^{0}$ emission initially predominant in the hydrogen-terminated material, Fu et al demonstrated that the $\mathrm{NV}^{-}$ratio is recovered by thermal etching in $\mathrm{O}_{2}$ at $465 \mathrm{C}$, as shown in figure $16(\mathrm{a}, \mathrm{b})$. Unsurprisingly the effect was more pronounced for the shallower defects. Oxygen termination can also be achieved with acid boiling, with equal ratios of perchloric, nitric and sulphuric acids commonly used [171, 167, 113], and generally a combination of both acid boiling and annealing in $\mathrm{O}_{2}$ is employed for the most effective termination [57, 172]. Fluorine has also been shown to terminate the surface effectively for $\mathrm{NV}^{-}$at $15 \mathrm{~nm}$ below the surface. Fluorine termination is accomplished through fluorocarbon plasmas such as $\mathrm{CF}_{4}[173,174]$. The study by Cui and $\mathrm{Hu}$ reported that it can be more effective than oxygen treatments, as shown in figure 16(c), owing to the fluorine atom's higher relative electronegativity to carbon and its ability to fully passivate the surface [112]. Fluorine is also reported to provide an air-stable surface chemistry for diamond, whilst the oxygen termination can show degradation on the adsorption of moisture.

\subsection{Coherence Properties of Colour Centres in Engineered Diamond}

For the construction of diamond-based nodes for quantum networks, a primary consideration is the coherence of the optical transitions and of the electron spin. There have been many reports suggesting that the NV spin-coherence typical of native NVs in bulk CVD material can be maintained upon nanostructure fabrication [52, 175, 129]. Where ion implantation techniques are used, high temperature annealing $(>1000 \mathrm{C})$ has been shown to be critical in extending the spin-coherence of these centres [137] by removing excess paramagnetic defects from the lattice. 

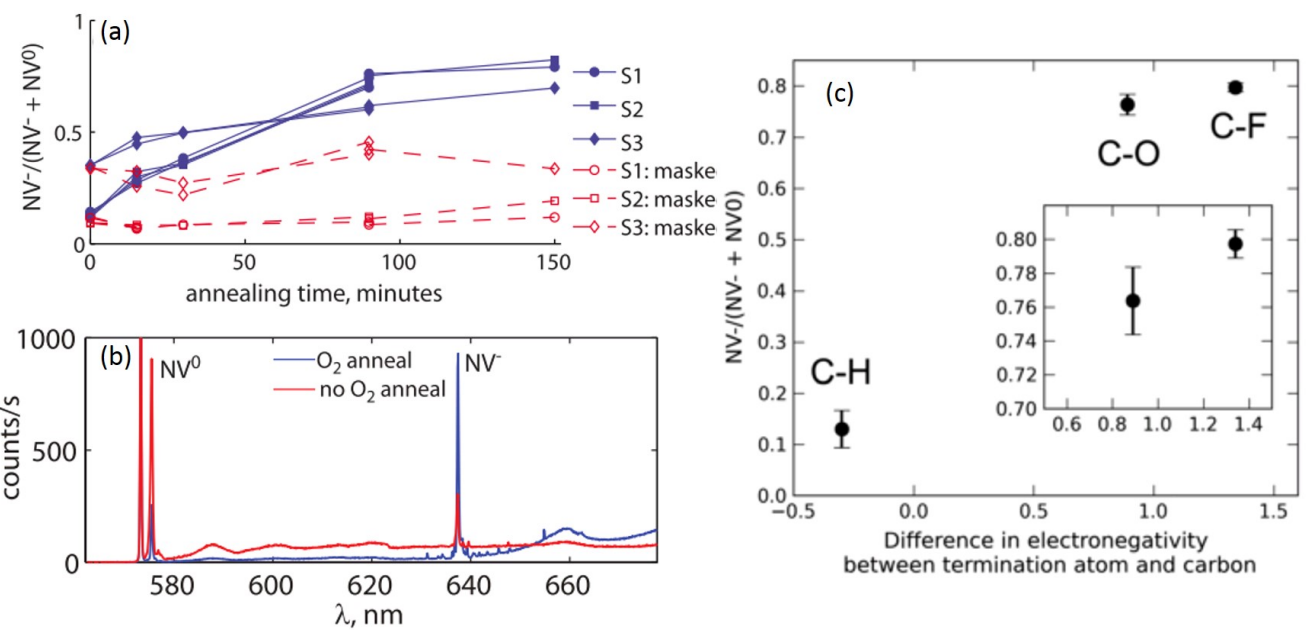

Figure 16: Influence of surface termination on the charge state of sub-surface NV (a) Samples S1, S2, S3 are initially hydrogenated and contain implanted NVs ensembles at 14, 14 and $62 \mathrm{~nm}$ respectively. The $\mathrm{NV}^{-}$to $\mathrm{NV}^{0}$ ratio is shown as a function of annealing time in an $\mathrm{O}_{2}$ atmosphere (blue). Some regions are masked as a control (red). (b) Low temperature PL spectra before (red) and after 150 min of $\mathrm{O}_{2}$ annealing (blue). Reprinted from [53], with permission from AIP Publishing. (c) The $\mathrm{NV}^{-}$to $\mathrm{NV}^{0}$ ratio for $\mathrm{NV}$ centres at $15 \mathrm{~nm}$ below the surface, plotted as a function of the electronegativity of the terminating species relative to carbon. Reprinted from [173], with permission from AIP Publishing.

However, there have been few reports of optically coherent transitions in photonic structures and membranes to meet the criterion of indistinguishability. Upon fabrication of PCCs into diamond in single crystal diamond through oxygen plasma RIE (detailed in section 4), Faraon et al presented PLE measurements on the cavity-coupled NV ZPL transitions, with linewidths of $\approx 4-9 \mathrm{GHz}$, orders of magnitude greater than the transform limit [51]. Mouradian and coworkers more recently reported PLE measurements of NV centres situated in free-standing diamond ridge waveguides (see section 4.4) fabricated through combined $\mathrm{Ar} / \mathrm{Cl}_{2}$ and oxygen plasma RIE. Significantly narrower single-scan widths were measured down to $\approx 400 \mathrm{MHz}$ on average [176], however this line width is still over one order of magnitude greater than the transform limit.

In 2014 Chu and coworkers [57] published a strategy for generating optically coherent NV centres using ion implantation, as summarised in figure 17(a). After the removal of surface damage due to polishing using first $\mathrm{Ar} / \mathrm{Cl}_{2}$ then $\mathrm{O}_{2} \mathrm{RIE}$ (section 3.1.1), [57, 88], ${ }^{15} \mathrm{~N}$ ions are implanted to a depth of $\approx 100 \mathrm{~nm}$ followed by a multi-stage annealing process in vacuum. An initial $400 \mathrm{C}$ bake-out stage [141] is followed by stages at $800 \mathrm{C}$ to initiate efficient $\mathrm{NV}$ formation and $1200 \mathrm{C}$ to heal the lattice damage $[142,125]$. Effective oxygen termination of the diamond surface is then established using a combination of tri-acid boiling and thermal oxidation (section 3.3). The resulting NV centres showed good optical properties at low temperature, with PLE measurements showing spectral diffusion to be almost eliminated with $\gamma+\gamma_{\text {inhom }}=27 \mathrm{MHz}$ (figure 17(c)). Equally impressive is the high fraction of NV centres created with this process that showed coherent optical properties, as evidenced by the statistics in the histogram of figure $17 \mathrm{~b}$. A similar methodology has been applied with artificially fabricated $\mathrm{SiV}$ centres via silicon ion implantation by Evans et al [141]. This work underscores the importance of the final annealing 


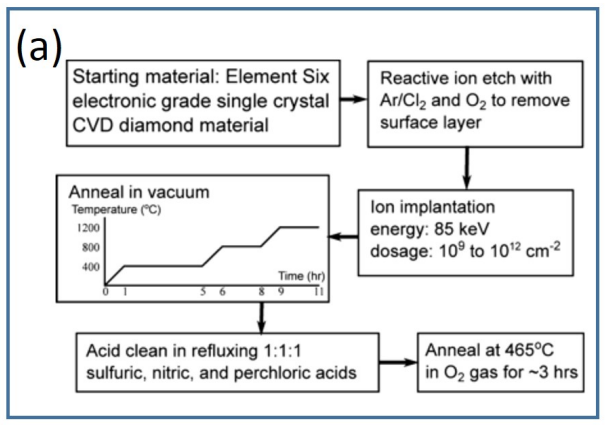

(c) Counts per second
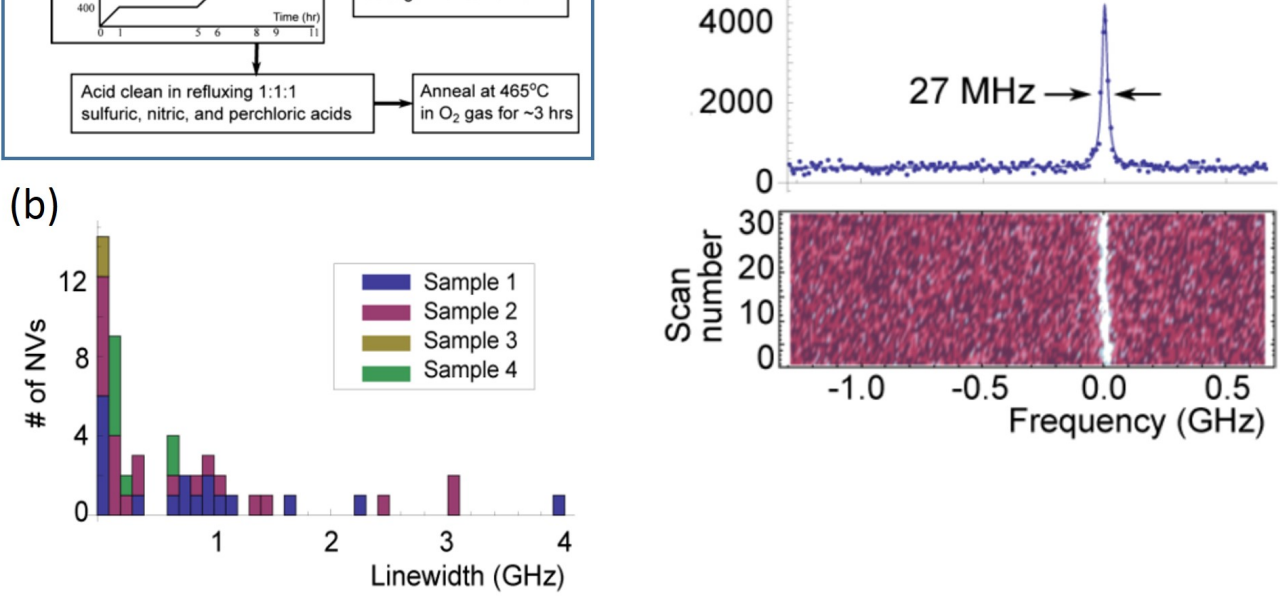

Figure 17: Generation of optically coherent sub-surface NV centres by ion implantation. (a) Protocol steps. (b) Histogram (100 MHz binning) summarising NV linewidths across four samples. (c) PLE spectrum averaged over repeated scans below with application of a $575 \mathrm{~nm}$ ( $\mathrm{NV}^{0}$ resonant) pulse for charge state recovery between each scan. Reprinted with permission from [57]. Copyright 2014 American Chemical Society. 
stage at $>1000 \mathrm{C}$, by showing the inhomogeneous distribution of separate $\mathrm{SiV}$ emission wavelengths to collapse from 3-4 nm after the $800 \mathrm{C}$ stage, to below $0.03 \mathrm{~nm}$ after the final stage. The coherent emission was demonstrated with PLE line widths of $320 \pm 180 \mathrm{MHz}$ across all centres studied, approaching the lifetime limit of $\gamma=94 \mathrm{MHz}$, for the transition of interest. These SiVs were also incorporated into free-standing waveguides through angular etching methods (see section 4.4). The line widths here remained largely unchanged at $410 \pm 160 \mathrm{MHz}$. These results suggest that coherent optical transitions from artificially generated colour centres incorporated into processed and nanostructured diamond is an achievable engineering challenge for the fabrication of photonic structures.

\section{Photonic Structures I: Fluorescence Collection and Waveguiding}

Advancing diamond processing methods have allowed for the fabrication and demonstration of a diverse range of photonic elements. The focus of this section is on non-resonant structures that enhance broad-band light collection efficiency $\eta_{c o}$ and provide methods for on-chip waveguiding. For enhanced light collection we discuss SILs, bullseye structures, nanowires and nanopillars. SILs in particular were an enabling factor in the recent remote spin-spin entanglement experiments between NV centres [11]. Expansion to larger-scale networks with NV centres will also require increases to $\eta_{e m}$ via resonant enhancement of the ZPL transition, discussed in section 5, but for colour centres such as the $\mathrm{SiV}$ with large Debye Waller factors, the non-resonant structures discussed in this section may provide sufficiently large $\eta_{c o}$ for scalability.

The second topic of this section will be progress towards integrated on-chip diamond photonic systems which have been facilitated by the processing methods of section 3. A tantalising longterm vision is for chip-based devices in which built-in photon routing elements, beamsplitters, switches and detectors allow fully scalable implementation of qubit networks [177]. Techniques that could be applied to the fabrication of on-chip photonic networks will be discussed, for example in the case of angular etching protocols of section 4.4. The engineering of these structures is also of importance to the discussion of integrated resonators in section 5.1, as these involve developments of the same basic architecture.

\subsection{Solid Immersion Lenses}

Solid immersion lenses (figure 18(c,d)) enhance light collection from sources in bulk materials by eliminating the refraction normally experienced at planar interfaces. The critical angle for total internal reflection at the diamond-air interface is $25^{\circ}$, so that only $4.5 \%$ of light emitted isotropically from a subsurface colour centre is transmitted through a planar interface and can be collected by a high numerical aperture lens (noting that at cryogenic temperatures oil immersion lenses are not practical). Light emitted by a defect at the centroid of a SIL, however, is normal to the surface over a solid angle approaching $2 \pi$, such that a SIL alone can increase $\eta_{c o}$ to nearly $50 \%$, an increase of an order of magnitude.

Other than the need to be compatible with high numerical aperture light collection optics, the diameter of a SIL has no direct impact on its functionality. One approach has been to fabricate 'macroscopic' SILs, of $500 \mu \mathrm{m}$ radius, made entirely out of diamond, through combined mechanical and laser processing [178]. Here the defect emission from the central position can be characterised both through the SIL and on the reverse side as a direct comparison of the enhancement, with over a factor of 6 observed. A more widely adopted approach has been to fabricate 
microscopic SILs via FIB milling [120]. These can be fabricated directly into the diamond surface using FIB milling into a region of type IIa diamond with a sufficiently low NV density, shown in figure $18(\mathrm{c})$. With the defects to be centered in a hemisphere of $2.5 \mu \mathrm{m}$ radius, the influence of surface states on the coherent properties of the centre is limited. Microscopic SILs are considerably more robust to shape imperfections than macroscopic SILs, since the degree of wavefront distortion of the escaping light depends on the absolute variation in path length from the emitter to the hemispherical surface. As well as enhancing emission, the magnifying power of the SIL (equal to the refractive index) reduces the excitation spot size, thus reducing the required excitation power. This can provide the additional benefit of reducing the intensity of background fluorescence and scattering relative to the colour centre fluorescence, important for achieving good single-photon fidelities. Finite-difference time-domain (FDTD) simulations, an example of which is shown in figure 18(d), suggest that the positional accuracy of the defects needs to be better than $1 \mu \mathrm{m}$ to ensure a significant enhancement of the collection efficiency [121]. Marseglia et al developed a registration technique to create SILs around pre-identified NV centres [122]. The lateral positioning accuracy of the fabricated SIL was determined to be $210 \mathrm{~nm}$, within the design tolerance for improved light collection. Enhancements of a factor of 8 were thus achieved. Further work on integrated SILs has seen photon count-rates of up to $10^{6} \mathrm{~s}^{-1}$ from a single NV centre in [111] oriented diamond [179]. SILs have also been used in conjunction with stimulated emission depletion microscopy of $\mathrm{NV}$ centres to eliminate aberrations in the excitation and depletion beams, resulting in a spatial resolution down to $2 \mathrm{~nm}$ and sub-nanometre position localisation [180]. Hybrid structures have been studied, whereby NV centers in a diamond membrane have been attached to the planar surface of a GaP SIL with 1 $\mathrm{mm}$ radius. Here the higher refractive index material $\left(n_{\mathrm{GaP}}=3.3\right)$, ensures that the vast majority of the fluorescence is preferentially directed through the SIL [181].

\subsection{Bullseye Structures}

Circular diffraction gratings etched into $300 \mathrm{~nm}$ membranes, shown in figure 18(a,b), serve to suppress emission in the plane and therefore provide strongly directional coupling to external collection optics. They have been shown to achieve the highest single NV photon count rates reported to date of $\approx 3 \times 10^{6}$ counts/s. The structures were fabricated using RIE through a silicon hard-mask mask (introduced in section 3.2.1). Hahn echo measurements showed $T_{2}$ in the region of $300 \mu \mathrm{s}$, whilst dynamical decoupling techniques (introduced in section 2.1) allowed the coherence time to be extended to $1.7 \mathrm{~ms}$ [175]. To get an indication of the requirements for the lateral positioning accuracy, the authors performed FDTD simulations suggesting that a $\approx 200 \mathrm{~nm}$ radial displacement of the emitter from the centre of the grating is enough to reduce the maximum collection efficiency by factor of two.

\subsection{Nanowires and Nanopillars}

Integrated nanowire and nanopillar structures provide another approach to achieving directional emission towards external collection optics, using the principle of waveguiding by the high index diamond. Nanopillars $(\approx 200 \mathrm{~nm}$ in length) and nanowires $(>1 \mu \mathrm{m})$ have both been explored [184, 183], whilst the single NV yield of these devices has improved with prior ion implantation and annealing [185]. This architecture is suitable for top-down processing, facilitating the creation of arrays as in reference [19]. Babinec et al [183] demonstrated a ten-fold 


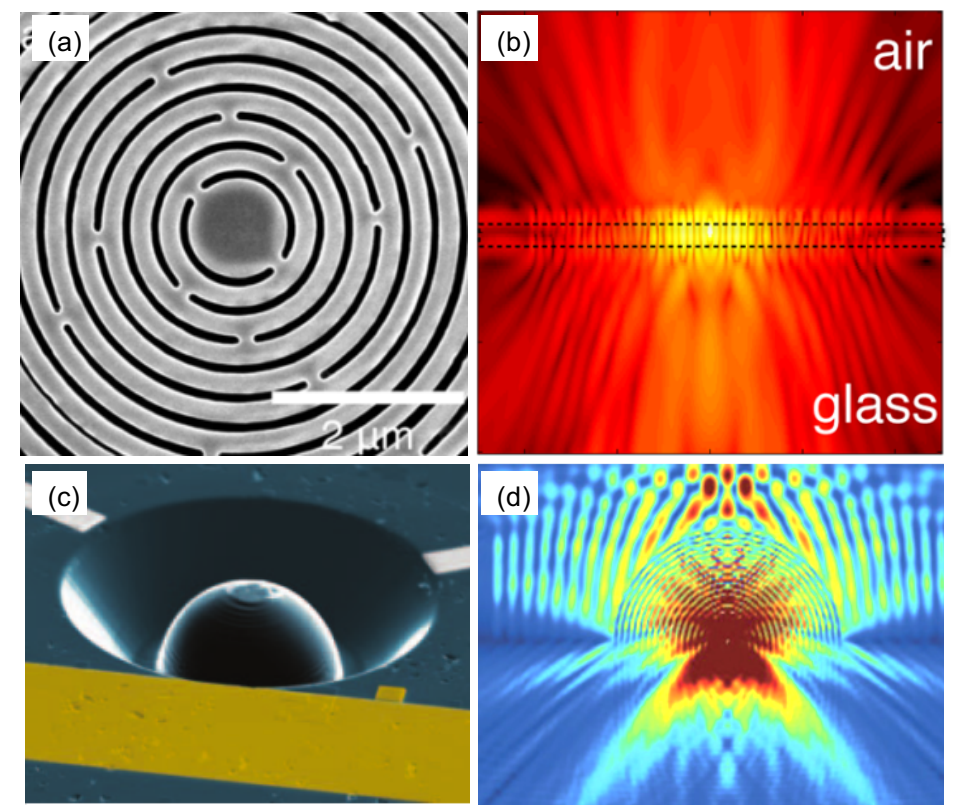

Figure 18: Structures to enhance light collection from colour centres in diamond (a) SEM Image of a bullseye structure in diamond. (b) FDTD simulation of the electric field intensity (log-scale). Reprinted with permission from [175]. Copyright 2015 American Chemical Society. (c) SEM image of a solid immersion lens milled into a diamond surface. Reprinted by permission from Macmillan Publishers Ltd: Nature [11], copyright 2013. (d) FDTD Simulation of the electric field intensity (log-scale). Reprinted from [121]. 

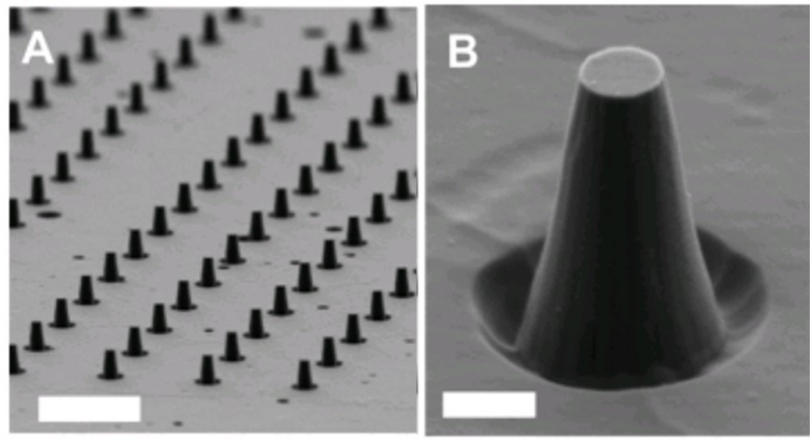

$\mathrm{cnts} / \mathrm{s}$

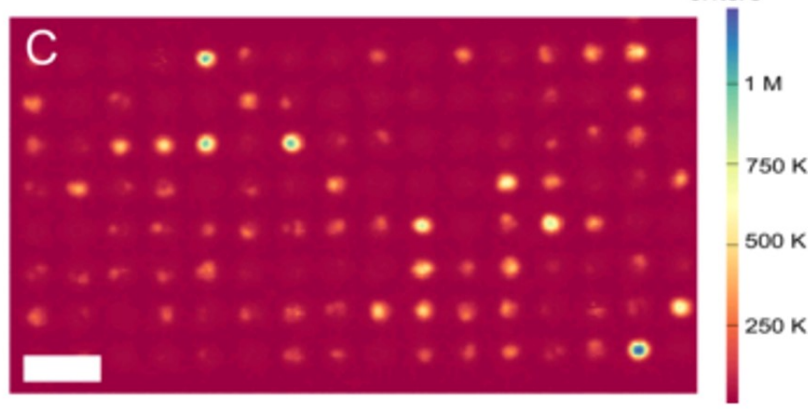

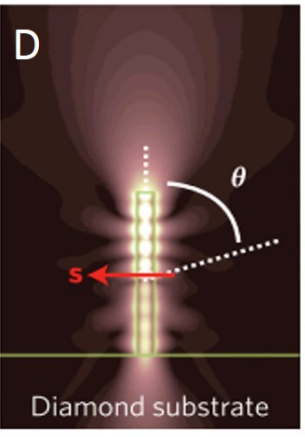

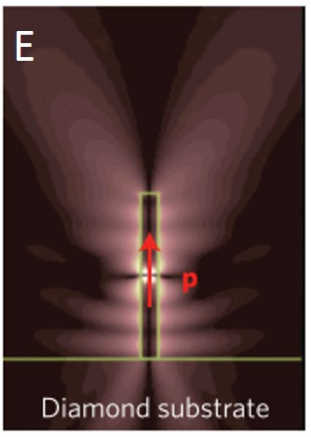

Figure 19: Nanowire/nanopillar structures in diamond. (a,b) SEM images of tapered diamond nanopillars with the scale bar denoting $3 \mu \mathrm{m}$. (c) PL image of a nanopillar array from the reverse side of the substrate showing single NV emission in excess of $10^{6}$ counts/s. Reprinted with permission from [182]. Copyright 2015 American Chemical Society. (d) Simulation of field profile for a radially oriented dipole in a diamond nanowire, with $\theta$ denoting an NA $=0.95$, typical of the best microscope air-objective lenses. The profile suggests optimal collection of light in the vertical direction. (e) Simulation of axially oriented dipole showing larger field components in the lateral directions. However, significant coupling to external optics is still achievable. Reprinted by permission from Macmillan Publishers Ltd: Nature Nanotechnology [183], copyright 2010. 
enhancement of the NV saturation photon count rates (>10 5 counts/s), with simultaneous 10-fold reduction in the saturation excitation power in the nanowire geometry compared with planar bulk light collection. The coupling efficiency is highly dependent on dipole orientation, with figure $19 \mathrm{~d}$ showing a simulation of the ideal coupling from an s-polarised dipole, whilst the sub-optimal p-polarisarion is shown in figure 19e. In (100) diamond substrates, the NV dipole emission has contributions from both of these components, resulting in a $40 \%$ collection efficiency by an objective lens in this geometry. With the advent of (111)-oriented CVD material [186, 127, 128], nanowires have seen further improvements in the achievable photon count rates to more than $10^{6}$ counts/s $[129,182]$. Tapering of the base, as in figure 19a,b has been shown to optimise this design still further [182]. These structures are well suited for applications in scanning magnetometry and as a consequence, significant efforts have been made to preserve the spin coherence of shallow (approaching $10 \mathrm{~nm}$ ) NV centres at the tip. With the appropriate processing (similar to those in section 3.3), it has been shown that the $T_{2}$ times of the starting materials are largely preserved upon nanostructuring [114, 182, 129], allowing for astonishing magnetic field sensitivities down to the single electron spin level [187]. Such developments are worth noting here as they could potentially open new possibilities for hybrid nodes in which an optically networked colour centre is scanned across an array of optically passive spin-qubits, thus greatly increasing the available resources in a single node.

\subsection{On-Chip Waveguides and Photonic Elements}

The combination of controlled top-down nanofabrication techniques for single crystal diamond and colour centre positioning provides the building blocks for development of integrated photonic circuits and on-chip networks. Early work towards an integrated platform focused on straightforward all-diamond ridge waveguide architectures [188, 189]. For full confinement of the optical mode however it becomes necessary to ensure a refractive index contrast between the waveguide and underlying material. A widespread approach has been to use deep RIE to fabricate diamond membranes, supported on $\mathrm{SiO}_{2} / \mathrm{Si}$ substrates, a few hundreds of nanometres in thickness, before fabricating photonic elements. The underlying $\mathrm{SiO}_{2}(n=1.45)$ provides sufficient refractive index contrast to confine the optical mode within the diamond. This approach has formed the basis of resonator architectures such as microrings $[102,69]$ which can be evanescently coupled to integrated waveguides and interrogated with via grating couplers [190, 103, 191]. Hybrid platforms based on gallium phosphide bonded to diamond present another approach to circumvent the issues facing all-diamond ridge structures, where the waveguiding occurs in the higher refractive index GaP layer. This system has yielded demonstrations of microdisks, microrings, directional couplers and grating couplers [192, 193, 194, 195, 196, 197, 198, 199] (discussed in section 5.1.3). The optimal confinement of modes to within the diamond material ultimately requires a free-standing architecture for best possible contrast. Within the limitations of top-down processing, some approaches have been taken to selectively etching the supporting substrate, commonly silicon, in order to suspend the photonic structures [52, 99, 200, 70]. High-energy ion bombardment of single crystal diamond followed by homoepitaxial overgrowth of high quality membranes provides another route towards membrane suspension as the damaged layer can be selectively etched [97, 93, 96, 92]. Micromanipulation and lift out of as-fabricated membranes $[93,94,92]$ could also be employed to enable a free standing structure, however this serial technique is naturally slower than parallel fabrication processes.

The development of angular RIE protocols, in which bespoke Faraday cage structures have been widely used to direct ions at oblique angles to the diamond surface [104], has provided an ingenious new method for producing photonic structures (figure 20). The technique has been 
(a)

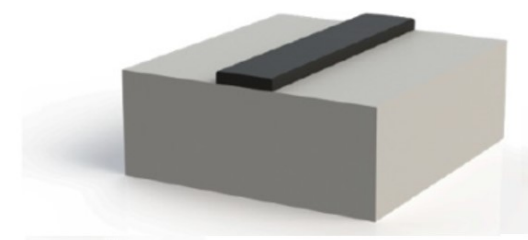

(c)

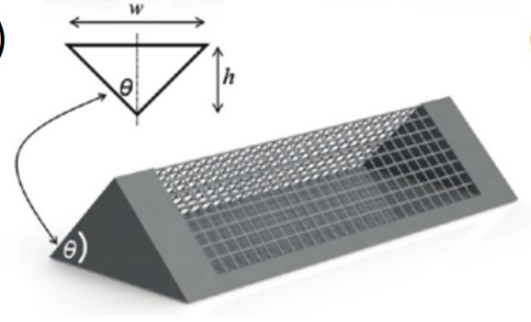

(b)

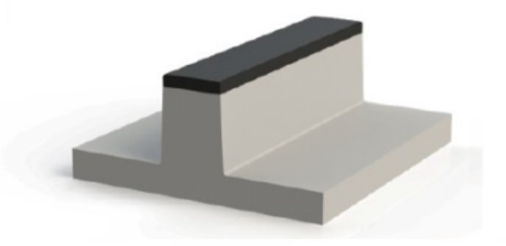

(d)

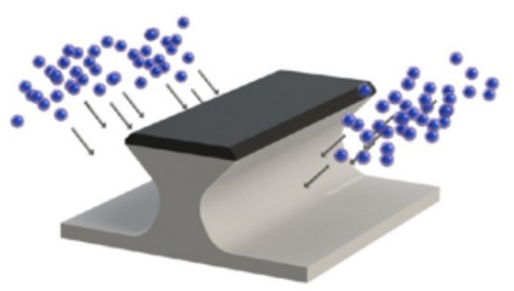

Figure 20: The angular etching protocol. (a) The diamond (light grey) is masked (dark grey) and removed (b) using a top-down etching protocol. (c) A Faraday cage is used to facilitate angular etching to undercut the masked feature as in (d). Reprinted with permission from [104]. Copyright 2012 American Chemical Society.

used to produce photonic crystal nanobeams, microdisks and waveguides [104, 201] and more recently racetrack resonators [87] (see section 5). The fabrication of free-standing devices directly onto the surface of the diamond releases the constraint of the membrane thickness on the operating wavelength of the device, in principle allowing multiple devices with distinct operating frequencies on one substrate which enables applications for on-chip frequency conversion [90]. Alternatively a quasi-isotropic plasma etch has more recently been developed to create undercut features in diamond, enabling high quality microdisks and nanobeam structures for optomechanics [202, 203]. Strategies towards the undercutting of photonic structures exclusively via FIB have been used to create nanobeam cavities using angled and side-milling [98, 124, 115], Q-factors of these devices were initially limited due to ion damage until later work by RiedrichMöller and coworkers showed that extensive post-processing steps with high temperature vacuum annealing, thermal oxidation and acid cleaning could start to recover the quality of the starting material and fabricate high-Q devices $[70,71]$.

\section{Photonic Structures II: Optical Resonators}

Optical microresonators can be employed to enhance and control light-matter coupling in a variety of ways [204]. We limit discussion here to the weak coupling regime of cavity quantum electrodynamics, which provides enhancement of spontaneous emission into well-defined modes for photonic networking, in particular for increasing the efficiency of the light-matter interface via $\eta_{e m}$ and $\eta_{c o}$. Schemes for quantum information processing involving solid state qubits strongly coupled to optical resonators have been proposed [205], but such 'quantum strong coupling' is considerably more difficult to realise than weak coupling and is not a required element of measurement-based network architectures.

In weak coupling the role of the resonator can be described in terms of its modification to the 
optical density of states experienced by the atomic system. The affect this has on the spontaneous emission process (the Purcell effect) is the most commonly cited benefit, but it is important to note that other schemes for spin-photon interfaces such as those of Hu et al [19] and Nemoto et al [20] benefit in similar ways from weak cavity coupling. Weak coupling occurs when the cavity photon leakage rate $\kappa$ is greater than the free-space atomic emission rate $\gamma$ and the atom-cavity coupling strength $g$, and substantial effects are observed when the single atom cooperativity $C=\frac{g^{2}}{\kappa \gamma}$ is of order unity or greater. Typical magnitudes for these parameters in diamond colour centres and microresonators are $\gamma \sim 0.1 \mathrm{GHz}, \kappa \sim 10-100 \mathrm{GHz}$, and $g \sim 0.1-10 \mathrm{GHz}$.

There are two principal ways in which optical resonators can enhance the efficiency for generation of indistinguishable photons beyond that which can be achieved by non-resonant structures. The first is by promoting emission of ZPL photons over those from phonon sidebands (enhancing $\eta_{e m}$ ), and the second is by increasing the homogeneous line width of the emitter (enhancing $\eta_{e x}$ ), a consequence of reducing the spontaneous emission lifetime via the Purcell effect. Both of these mechanisms are likely to be significant in the use of cavities for NV centres, due to the low Debye Waller factor and the sensitivity of the ZPL to inhomogeneous broadening mechanisms. The reduced optical power required for resonant excitation of cavity-coupled transitions may in addition improve fidelity by reducing background noise.

Before reviewing progress in the realisation of cavity-coupled structures, we outline the basic theory that describes the increase in ZPL emission efficiency and homogeneous linewidth. Our starting point is the enhancement of the rate of emission by a narrow atomic transition into a resonant cavity mode. This enhancement is given by the ratio of the optical density of states experienced by an atom in a resonant cavity mode compared to that in free space, encapsulated by the Purcell factor, $F_{\mathrm{P}}$ :

$$
F_{\mathrm{P}}=\frac{3}{4 \pi^{2}}\left(\frac{\lambda}{n}\right)^{3} \frac{Q}{V_{\text {mode }}}
$$

where $\lambda$ is the emitter-cavity resonance, $n$ is the refractive index, $Q$ is the cavity quality factor and $V_{\text {mode }}$ is the mode volume. This equation assumes that the emitter and the cavity mode are in perfect resonance, that the emitting dipole is situated at the electric field maximum of the cavity mode and is oriented parallel to the field direction, and that the atomic line width is narrower than the cavity mode line width (ie the dephasing time $\gamma *<\kappa$ ). The generalised form of the Purcell factor:

$$
F_{c a v}=F_{\mathrm{P}}\left(\frac{|\underline{\mu} \cdot \underline{\mathcal{E}}|}{|\underline{\mu}| \underline{\mathcal{E}_{\mathrm{max}}} \mid}\right)^{2} \frac{1}{1+4 Q_{\mathrm{cav}}^{2}\left(\lambda_{\mathrm{em}} / \lambda_{\mathrm{cav}}-1\right)^{2}}
$$

accounts for both spatial and orientational misalignment of the emitter dipole vector $\mu$ and the cavity mode electric field vector $\mathcal{E}_{\max }$ and relative spectral detuning between the emitter $\lambda_{\mathrm{em}}$ and

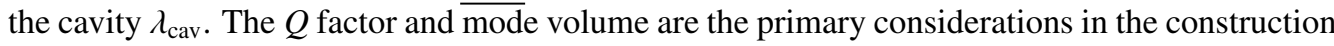
and design of a cavity system. For the implementation of an emitter-cavity system however, the position, orientation and resonance with the emitter become critical.

The effect of the cavity on $\eta_{e m}$ is to enhance emission into the desired mode relative to competing mechanisms for relaxation from the optically excited state. In diamond colour centres these include both other radiative processes such as phonon sideband (PSB) emission, and nonradiative (NR) processes. Typically diamond colour centres, like other emitters, will relax from 
the optically excited state via both radiative and non-radiative channels. The radiative emission can in turn be separated into the ZPL and photon sideband contributions:

$$
\gamma=\gamma_{r a d}+\gamma_{n r}=\gamma_{Z P L}+\gamma_{P S B}+\gamma_{n r}
$$

The ratio of radiative emission to the total relaxation rate $\gamma_{\text {rad }} / \gamma$ is known as the quantum efficiency of the emitter. If the ZPL is transform limited, the efficiency of emission of indistinguishable photons in bulk material is $\eta_{e m}=\gamma_{Z P L} / \gamma$

Coupling the ZPL emission to a microcavity mode enhances the ZPL emission rate by $F_{P}$. There may also be some effect of the microcavity on the phonon sideband emission, which can be partially suppressed by a reduced optical density of states [206, 71]. In general the total emission rate of the cavity-coupled colour centre will be

$$
\gamma^{\prime}=\gamma_{r a d}+\gamma_{n r}=F_{c a v} \cdot \gamma_{Z P L}+\beta \gamma_{P S B}+\gamma_{n r}
$$

where $0<\beta<1$ represents the suppression of the sideband emission. The efficiency of emission of the ZPL photons is now given by

$$
\eta_{e m}{ }^{\prime}=\frac{F_{c a v} \cdot \gamma_{Z P L}}{\gamma^{\prime}}=\frac{F_{c a v} \cdot \gamma_{Z P L}}{F_{c a v} \cdot \gamma_{Z P L}+\beta \gamma_{P S B}+\gamma_{n r}}
$$

For large enough $F_{P}$ this efficiency can approach unity. For example in $\mathrm{NV}^{-}, \gamma_{Z P L} \simeq 3 \mathrm{MHz}$, $\gamma_{P S B} \simeq 80 \mathrm{MHz}$ and we neglect $\gamma_{n r}$. In the absence of a cavity, $\eta_{e m} \simeq 0.04$ but in a cavity with $F_{P}=100$ one achieves $\eta_{e m}=0.79$, and $F_{P}=1000$ gives $\eta_{e m}=0.97$ (assuming conservatively that $\beta=1$ ). One can see from these examples that high efficiencies can be achieved with realisable microcavities. Equation 2 shows that for a cavity of mode volume $V=\lambda^{3}$ the Purcell factor is of order $F_{P} \simeq Q / 10$.

The increase in excitation efficiency $\eta_{e x}$ gained by increased homogeneous broadening is more subtle. For a fixed wavelength of excitation laser, the efficiency with which the colour centre will be excited is approximately the ratio of the homogeneous to total line width $\eta_{e x}=$ $\gamma /\left(\gamma+\gamma_{\text {inhom }}\right)$. For the transform limited ZPL the homogeneous line width is the same as the total emission rate $\gamma$, which changes to $\gamma^{\prime}$ in the cavity. Thus for an NV centre in which the phonon sideband emission rate is unchanged by the cavity, we find that $F_{P}=100$ gives $\gamma^{\prime} / \gamma=$ 4.6 and $F_{P}=1000$ gives $\gamma^{\prime} / \gamma=37$. For inhomogeneous broadening of $\gamma_{\text {inhom }}=300 \mathrm{MHz}$ the excitation efficiency without a cavity would be $\eta_{e x} \simeq 21 \%$, rising to $55 \%$ in a cavity of $F_{P}=100$ and $91 \%$ for $F_{P}=1000$.

With these basic considerations in place, we now turn attention to the main strategies adopted so far for cavity coupling of diamond colour centres. We begin by reviewing work on integrated diamond photonics approaches with all-diamond resonators (section 5.1) and hybrid diamondGaP structures (section 5.1.3). We then look at recent work on coupling to open-cavity systems (section 5.2). Throughout we focus on the particular challenges of the use of optical resonators to enhance the spin-photon interface, considering the achievable Purcell factors when coupling to zero phonon lines of colour centres. Other important considerations are the degree to which the ZPL-cavity coupling can be achieved on-demand, including reproducibility and in-situ tuning that will be highly advantageous in any scalable system (discussed in section 3). Efforts towards optimal defect placement within these photonic structures will also be highlighted. 


\subsection{Integrated Resonators}

\subsubsection{Photonic Crystal Cavities (PCCs)}

A photonic crystal consists of a periodic dielectric structure, serving to create a photonic band-gap, prohibiting the propagation of light of particular wave vectors. To form a PCC one or more of these 'lattice sites' are modified or removed forming a local modification of the band structure (a 'photonic crystal defect') that allows light to be confined [204]. Both onedimensional (referred to as nanobeam cavities) and two dimensional PCC architectures have been used to enhance emission from colour centres in diamond. Early demonstrations took a hybrid approach, whereby 2D-PCCs were fabricated from GaP, with pick-and-place techniques employed to position NV centres in nanodiamond at the cavity mode [207, 208, 209]. However the developments in single-crystal diamond fabrication have allowed for monolithic structures, where periodicity is achieved through an array of air-holes in membrane thickness material, as in figure 21(a). The high-refractive index contrast between air and diamond means the penetration of the field into the surrounding crystal is low, leading to ultra-small mode volumes of the order $V_{\text {mode }} \approx\left(\frac{\lambda}{n}\right)^{3}$. Though FDTD simulations suggest these structures are capable of sustaining high$\mathrm{Q}$ modes [210,211], the challenge is to maintain these properties in fabrication, which depends strongly on the ability to obtain smooth, vertical side-walls for the air-holes.

Riedrich-Möller and co-workers [70, 71] have pioneered the FIB approach, demonstrating both 2D-PCC and nanobeam structures with mode volumes of $V_{\text {mode }}=1.5\left(\frac{\lambda}{n}\right)^{3}$ (2D PCC) and $V_{\text {mode }}=0.7\left(\frac{\lambda}{n}\right)^{3}$ (Nanobeam) respectively and Q-factors up to 700. These Q-factors are at least one order of magnitude less than the idealised structures, a limit which is shown to be primarily due to the side-wall tapering, but nevertheless provide Purcell factors of $F_{P} \simeq 35$. The as-fabricated cavity modes are spectrally very close to the targeted colour centre resonances, red-shifted just a few $\mathrm{nm}$. Tuning of the cavity resonances was achieved through repeated thermal oxidation to reduce the structure dimensions, showing spectral blue-shifting of the cavity mode by up to $15 \mathrm{~nm}$ without significant degradation of the Q-factor. SiV ensembles were used to trial the emitter-cavity coupling process with the 2D-PCC showing a factor of 2.8 intensity enhancement at the ZPL wavelength for light collected above the plane.

One of the most significant demonstrations of 2D-PCC structures in diamond was by Faraon and co-workers [51] who fabricated PCC structures using $\mathrm{O}_{2}$ RIE and demonstrated low temperature coupling of single NVs to the cavity modes with some of the highest Purcell factors to date. Here a PCC with three missing lattice points (categorised as an M3 cavity), offered a $V_{\text {mode }}=0.88\left(\frac{\lambda}{n}\right)^{3}$. Q-factors of $\approx 3000$ were shown, about half of the simulated value, corresponding to a Purcell factor of $F_{P}=260$. Coupling to the ZPL of a single NV centre was demonstrated at $<10 \mathrm{~K}$. Here tuning of the as-fabricated cavity resonance to the ZPL is achieved by the condensation of xenon gas on the surface of the cold cavity structure, thus red-shifting the mode resonance with fine control by as much as $7 \mathrm{~nm}$ [212]. Lifetime measurements on the cavity coupled ZPL showed a factor of 3 reduction on resonance as shown in figure 21(b). Taking into account the Debye-Waller factor of 0.03 , this lifetime reduction corresponds to $F_{c a v}=70$. Single photon emission from the cavity-coupled ZPL was verified with a Hanbury Brown and Twiss (HBT) measurement, with $g^{(2)}(0)=0.38$.

Nanobeam cavities have also been fabricated with $\mathrm{O}_{2}$ RIE using the Faraday cage technique discussed in section 4.4. This process was first reported by the Lončar group at Harvard in 2012 [104], who have since fabricated cavities with $Q$-factors up to 8200 and mode volumes down to $V_{\text {mode }}=2.26\left(\frac{\lambda}{n}\right)^{3}\left(F_{P}=280\right)$ at visible wavelengths [87]. A different approach reported by the same group fabricates nanobeam structures from diamond membranes $(\approx 250 \mathrm{~nm})$ on silicon 


\section{(a) FIB Milled M7 Photonic crystal}

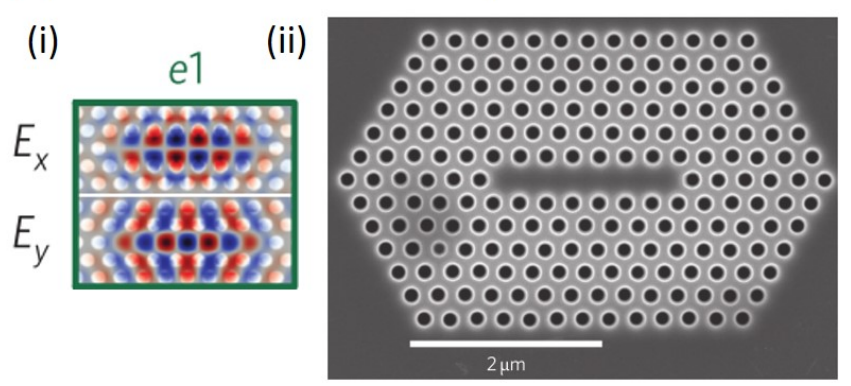

(b) $\mathrm{O}_{2}$ etched M3 Photonic Crystal

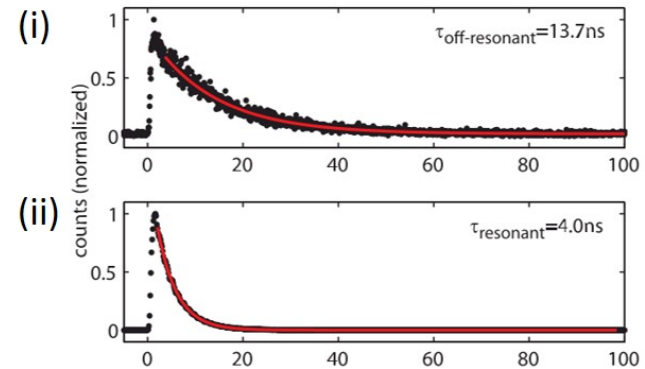

Figure 21: 2D photonic crystal cavity in diamond. (a) A FIB milled M7 (seven-hole defect) photonic crystal, (i) FDTD simulations of the $\mathrm{E}_{x}$ and $\mathrm{E}_{y}$ profiles of the fundamental e1 mode, and (ii) SEM image of the fabricated cavity structure. Reprinted with permission from Macmillan Publishers Ltd: Nature Nanotechnology [70], copyright 2012. (b) Data from a RIE etched M3 (three hole defect) photonic crystal. Lifetime measurements of a single NV centre when its ZPL is (i) off and (ii) on resonance with the photonic crystal mode, corresponding to a factor of $\approx 70$ enhancement of the ZPL when accounting for the branching ratio. Reprinted with permission from [51]. Copyright 2012 by the American Physical Society.

(a)

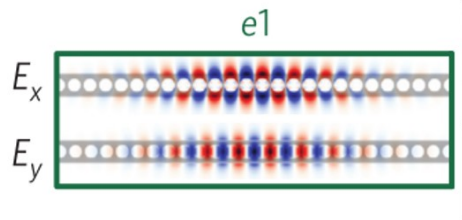

(b)

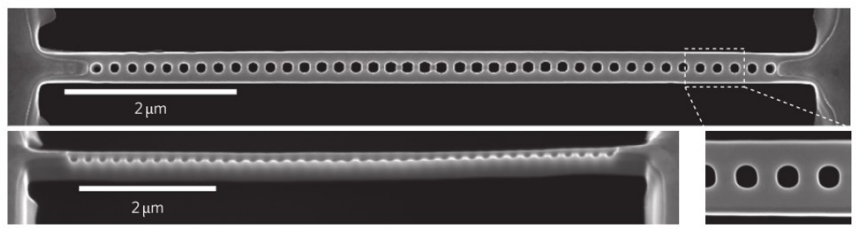

Figure 22: FIB milled suspended nanobeam cavities in diamond. (a) FDTD simulations of the $\mathrm{E}_{x}$ and $\mathrm{E}_{y}$ profiles of the fundamental e1 mode of the nanobeam cavity. (b) SEM image. Reprinted with permission from Macmillan Publishers Ltd: Nature Nanotechnology [70], copyright 2012. 


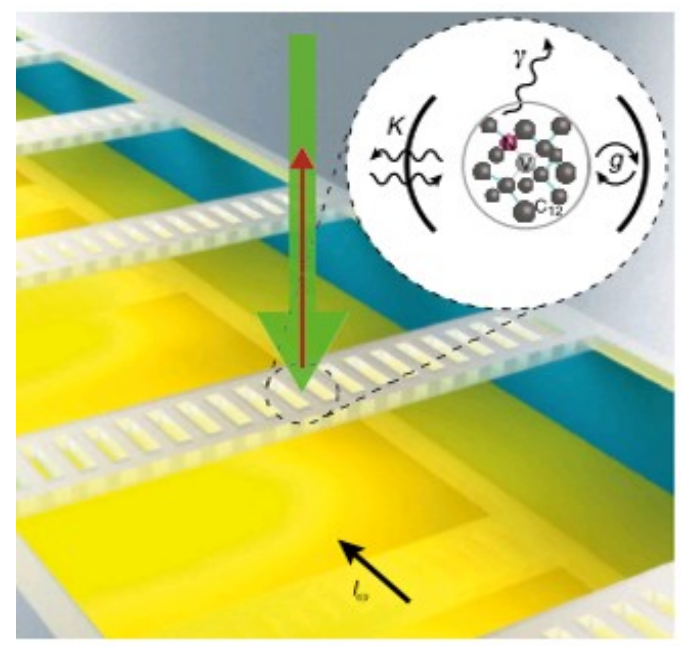

Figure 23: Photonic crystal nanobeam cavities - illustration of suspended nanobeam structures fabricated through silicon hard-masked RIE. The device is integrated with a microwave stripline for coherent spin control of the cavity coupled NV. Reprinted by permission from Macmillan Publishers Ltd: Nature Communications [52], copyright 2015.

substrates. E-beam patterning in photoresist is followed by $\mathrm{O}_{2} \mathrm{RIE}$ to transfer the PCC pattern into the diamond membrane, before using a final isotropic etch acting only on the supporting silicon to result in free-standing nanobeam structures [200]. Q-factors up to 6000 were reported, and both red $(7 \mathrm{~nm})$ and blue $(23 \mathrm{~nm})$ shifting of the cavity resonances were achieved using Xe condensation and thermal oxidation respectively. Coupling to a single NV centre at $4 \mathrm{~K}$ was demonstrated with a factor of 7 enhancement in the ZPL peak intensity on-resonance. This particular structure had $V_{\text {mode }}=3.7\left(\frac{\lambda}{n}\right)^{3}$ and $Q \approx 1600$.

Recently nanobeams fabricated by Li et al [52] have shown impressive Purcell factors when coupled to a single $\mathrm{NV}$ at $18 \mathrm{~K}$. An initial deep etch of alternating $\mathrm{O}_{2}$ and $\mathrm{Ar} / \mathrm{Cl}_{2}$ plasma recipes (section 3.3) was used to create a diamond membrane of $\approx 200 \mathrm{~nm}$ thickness with smooth surfaces. Silicon hard-masking of a further $\mathrm{O}_{2}$ plasma etch was then used for pattern transfer (section 3.2.1). Here Q-factors up to 9900 were demonstrated with $V_{\text {mode }}=1.05\left(\frac{\lambda}{n}\right)^{3}\left(F_{P} \simeq 720\right)$. Coupling of a single NV centre with a measured Debye-Waller factor of 0.02 to a $Q=3300$ nanobeam showed an almost three-fold reduction in the lifetime on-resonance, corresponding to $F_{\text {cav }}=62$. This device was integrated with a microwave-stripline resonator on silicon as shown in figure 23, allowing for measurements of the spin coherence and revealing $\mathrm{T}_{2} \simeq 230 \mu$ s suggesting that the spin coherence remains relatively unaffected by the fabrication of the resonator [52]. This is an important result, and one of the only reports to date of cavity-coupled spin-control in diamond, obtaining both a large Purcell factor and long spin coherence time, thus bringing together some of the major elements needed for the development of efficient quantum network nodes based on NV centres.

\section{Colour centre placement in PCCs}

The single colour centre coupling experiments discussed above have mostly relied on stochastic approaches and post-selection of cavity structures to obtain single colour centres in proximity 


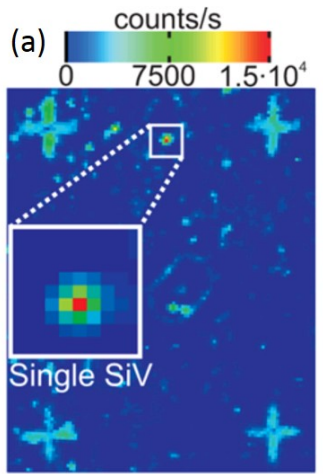

(b)

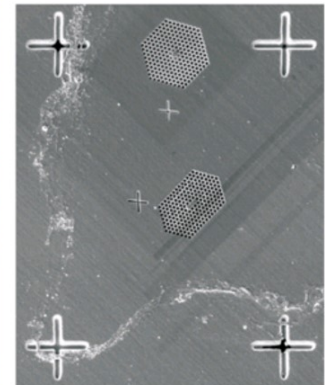

(c)

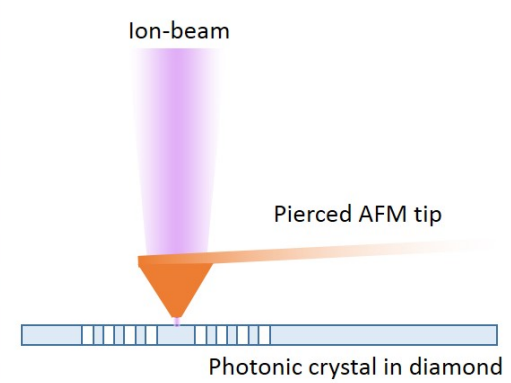

Figure 24: Strategies for incorporating defects with integrated devices. (a,b) A pre-registration strategy: (a) PL image of an identified single emitter (b) SEM image of photonic crystal cavities milled around the defects with registration markers for guidance. Reprinted with permission from [71]. Copyright 2014 American Chemical Society. (c) A pierced AFM tip collimates a focused nitrogen ion beam which can be targeted at an integrated device, in this case a 2D-PCC. Adapted from [72].

to the cavity mode. A scalable technology will look to increase the yield of successful emittercavity structures through a more deterministic approach. Riedrich-Möller et al [72] have used a pierced AFM tip, with a $30 \mathrm{~nm}$ aperture (introduced in section 3.2.1) to mask nitrogen ion implantation into M1, M3 and M7 PCCs in diamond. The implantation energy of $5 \mathrm{keV}$ was chosen to achieve transverse spatial resolution of $<15 \mathrm{~nm}$ combined with an implantation depth of $8 \pm 3 \mathrm{~nm}$. Coupling to NV centres was demonstrated in the M1 cavity, however the ion fluences used were not sufficiently low to reach the single NV limit. Lee and coworkers [97] used deltadoping (section 3.2.3) combined with a $10^{14} \mathrm{~cm}^{-2}$ dose of $2 \mathrm{MeV}$ electrons before annealing [167] to place ensembles of NVs in in photonic stuctures. The NV layer was situated $100 \mathrm{~nm}$ below the surface of the crystal and after nanobeam fabrication, cavities with minimum mode volumes $V_{\text {mode }}=0.47\left(\frac{\lambda}{n}\right)^{3}$ and Q-factors up to 24,000 were demonstrated $\left(F_{P} \simeq 3900\right)$. Lifetime studies at $4 \mathrm{~K}$ revealed $F_{c a v}=22$ when coupled to a $Q=7000$ structure, the discrepancy explained as resulting from the large number of NVs coupled to each cavity.

Deterministic construction of M1 and M7 PCCs around pre-registered single SiV centres was reported by Riedrich-Möller et al, as seen in figure 24a,b [71]. Coupling of a single SiV to a $V_{\text {mode }}=1.7\left(\frac{\lambda}{n}\right)^{3}$ and $Q=430$ cavity was demonstrated, attaining a 19 -fold enhancement of the measured ZPL fluorescence intensity at room temperature. HBT measurements elucidate the internal dynamics before and after cavity fabrication, showing only a 2.5 -fold reduction of the fluorescence lifetime attributed to the low quantum efficiency of the $\mathrm{SiV}$. The spatial mismatch between emitter and cavity also reduces the expected Purcell enhancement by $60 \%$ with respect to the idealised case.

Recent work by Schukraft and coworkers explored the integration of colour centre placement with the nanobeam fabrication process [149]. In this work, silicon hard-masks (introduced in section 3.2.1) served a dual purpose, both for pattern transfer to fabricate the PCC and to mask ion implantation, thereby reducing the number of lithographic steps. In addition to the larger holes for photonic crystal fabrication, the silicon mask had a $40 \mathrm{~nm}$ aperture aligned with the center of the cavity. The high-aspect ratio of this $40 \mathrm{~nm}$ aperture meant that the diamond did 


\section{(a) nanobeam $\longrightarrow$ tapered fiber}
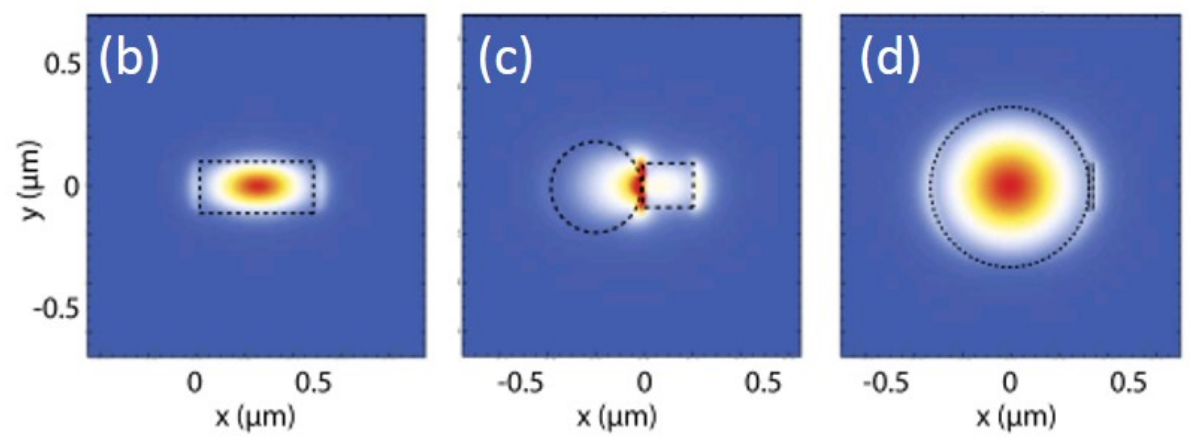

Figure 25: (a) Out-coupling scheme for nanobeam cavities. The tapered nanobeam waveguide is coupled to a tapered fiber. (b-d) FDTD simulations of the mode intensity from the (b) waveguide through to the (c) interface and the (d) optical fibre, showing the evolution and transfer of the mode. Reprinted with permission from [214]. Copyright 2015 Optical Society of America.

not etch efficiently at this position, but $100 \mathrm{keV}$ nitrogen ions penetrated it effectively and were implanted at a depth of $110 \mathrm{~nm}$ with anticipated straggle of $20 \mathrm{~nm}$. Following earlier efforts [201, 104], angular etching then yielded free standing nanobeams with triangular cross-sections. The single NV-cavity yield was estimated to be an impressive $26 \%$ and some initial room temperature NV coupling data were presented.

Out-coupling from PCCs

Of the PCCs, the nanobeam resonators are particularly well-suited to achieving efficient outcoupling of photons (high $\eta_{c o}$ ). Modulating the size of the periodic holes away from the centre of the resonator allows for efficient coupling from the cavity to the waveguide mode [70, 213]. By using tapered nanobeam architectures in conjunction with tapered fibers, these photons can then be connected to a wider photonic network, off-chip. This was demonstrated in principle with silicon-nitride ridge waveguides by Tiecke and co-workers, reporting coupling efficiencies up to $97 \%$ [214], shown schematically in figure 25a. Gradual tapering in the propagation direction enables the mode to be contained within the hybrid waveguide as shown in figure $25 \mathrm{~b}-\mathrm{d}$, whereby the mode is adiabatically transferred between the two optical structures. Recently, tapered diamond ridge waveguides were coupled to SiN integrated waveguide architectures using a pick-and-place method, with $10^{6}$ counts/s out-coupling from a single NV in the waveguide [176]. The out-coupling of NV waveguided emission to a tapered single mode optical fiber has also been shown with an efficiency of 16-37\% [215]. Whilst the first experimental values for $\eta_{c o}$ from a single emitter to a network are still awaited, these demonstrations show that efficient coupling to optical fibre systems should be possible. 


\subsubsection{Whispering Gallery Resonators}

Another popular approach to microcavitation is the use of whispering gallery mode resonators, relying on the total internal reflection at the interface to build up circulating modes [204]. This umbrella term encompasses microsphere, microtoroid, microdisk and microring structures, many of which have been demonstrated in principle through hybrid approaches with NV centres in nanocrystals or pillars coupled evanescently to non-diamond resonators [216, 217, 218, $219,220,221,195]$. Exploiting the maturing chip-based fabrication methods, whispering gallery resonators have found application within integrated diamond photonics. A prominent work on diamond microrings was conducted by Faraon et al [102], fabricating resonators from $280 \mathrm{~nm}$ diamond membranes with a $4.3 \mu$ m outer diameter, situated on an $\mathrm{SiO}_{2}$ substrate to confine the mode in the vertical plane (section 4.4), as shown in figure 26a. These structures have larger mode volumes than PCCs, with mode volumes in a range $V_{\text {mode }}=17-32\left(\frac{\lambda}{n}\right)^{3}$ and $Q$ up to 5000 $\left(F_{P} \simeq 20\right)$, limited by surface roughness. Low temperature coupling experiments demonstrated resonant coupling to the NV ZPL. Fabrication of the resonator incorporated strain into the structure, such that the initial high density of $\mathrm{NV}$ defects became individually resolvable spectrally. The device supports a plethora of resonances. Modes $\mathrm{C} 1$ and $\mathrm{C} 2$, as labeled in figure 26b, are identified as two counter propagating modes of the ring resonator with $Q=4300$ and 3800 respectively, which were tuned through the ZPL resonances using the gas-condensation technique. Lifetime measurements of the NV ZPL transition were taken as a function of mode detuning (figure 26c) corresponding to more than a ten-fold enhancement of the ZPL transition intensity.

More recently, racetrack resonators have been fabricated by the Lončar group in Harvard [87] using angular etching methods, with high $Q$ devices shown for both the visible $\left(Q_{t o t}=5.9 \times 10^{4}\right)$ and telecoms wavelengths $\left(Q_{t o t}=1.5 \times 10^{5}\right)$. Monolithic microdisk resonators have also been fabricated directly into single-crystal diamond through a quasi-isotropic plasma etching method [222]. These exhibited similarly high $Q=1.1 \times 10^{5}$ and $V=11\left(\frac{\lambda}{n}\right)^{3}\left(F_{P} \simeq 760\right)$ measured for $\lambda$ $=1500 \mathrm{~nm}$. However, colour-centre coupling was not implemented in these works.

\section{Out-coupling from WGM resonators}

Out-coupling of photons from whispering gallery modes generally relies on coupling to adjacent waveguides via the evanescent field surrounding the resonator. Such an architecture was demonstrated in diamond by Hausmann et al (figure 27a) [103]. In this work, isolated resonators were shown to have an intrinsic $Q$ up to 12600 , whilst the overall waveguide coupled device had $Q_{t o t}=3200$. A single NV coupled to a resonator was demonstrated, with the photons collected at grating out-couplers at the ends of the waveguide, and a measured efficiency of $\eta_{c}=$ 0.1 . Multiple resonances of the microring can be seen coupling to the PSB emission in figure 27b. Subsequently Faraon and coworkers also studied the coupling of the microring resonator geometry to integrated diamond ridge waveguides. Resonant enhancement of the NV ZPL was shown at cryogenic temperature, with most of the ZPL emission out-coupled from the grating terminated waveguide [190]. Transmission measurements indicate $Q_{t o t}=5500$, compared to $Q$ $=1.2 \times 10^{4}$ for an isolated microring. This waveguide coupled resonator system is almost at the critical coupling regime where the intrinsic loss rate and the coupling rate are balanced. Low temperature Purcell enhancements of up to 12 were demonstrated.

\subsubsection{Hybrid GaP/Diamond Devices}

Hybrid gallium-phosphide/diamond platforms have been developed as an approach to the outcoupling of NV emission from chip-based integrated architectures. With a bulk semiconductor 
(a)

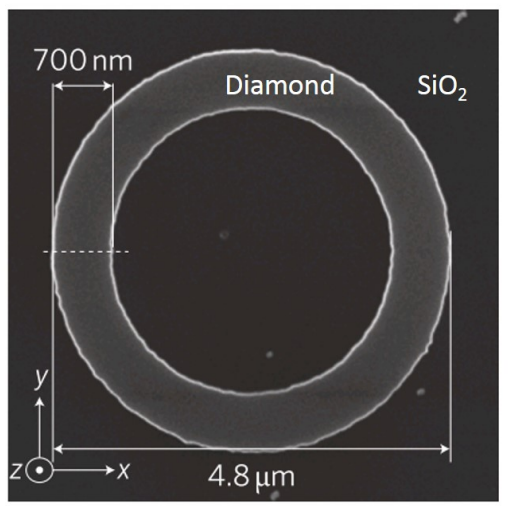

(b) $\mathrm{C}_{2} \mathrm{Cl} \quad \mathrm{NV} 1$

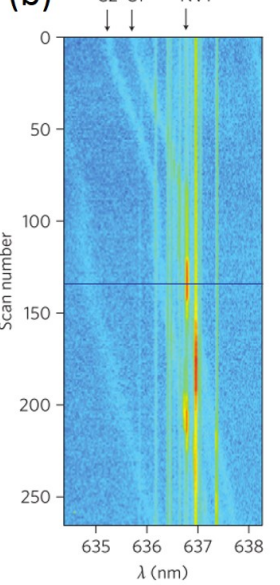

$\Delta \lambda_{1}(\mathrm{~nm})$

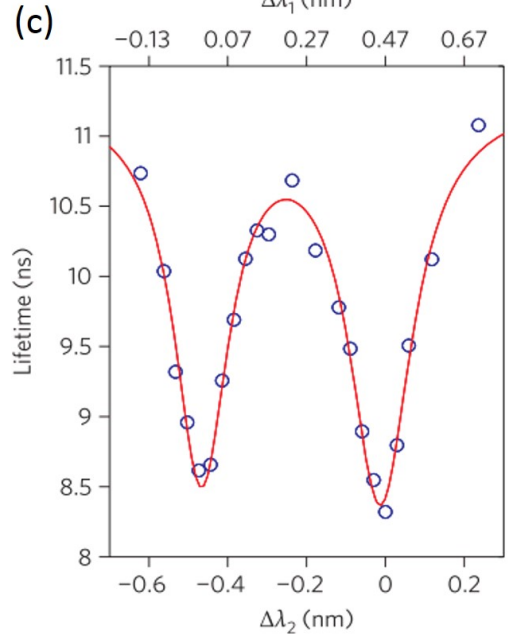

Figure 26: Microring resonators in single crystal diamond (a) SEM image of diamond microring on an $\mathrm{SiO}_{2}$ substrate (b) Tuning of the counter-propagating modes $\mathrm{C} 1$ and $\mathrm{C} 2$ through the ZPL of the NV centres at cryogenic temperature. The ZPL of NV1 is indicated. (c) The lifetime of NV1 as C1 and C2 are tuned through resonance. Reprinted with permission from Macmillan Publishers Ltd: Nature Photonics [102], copyright 2011.
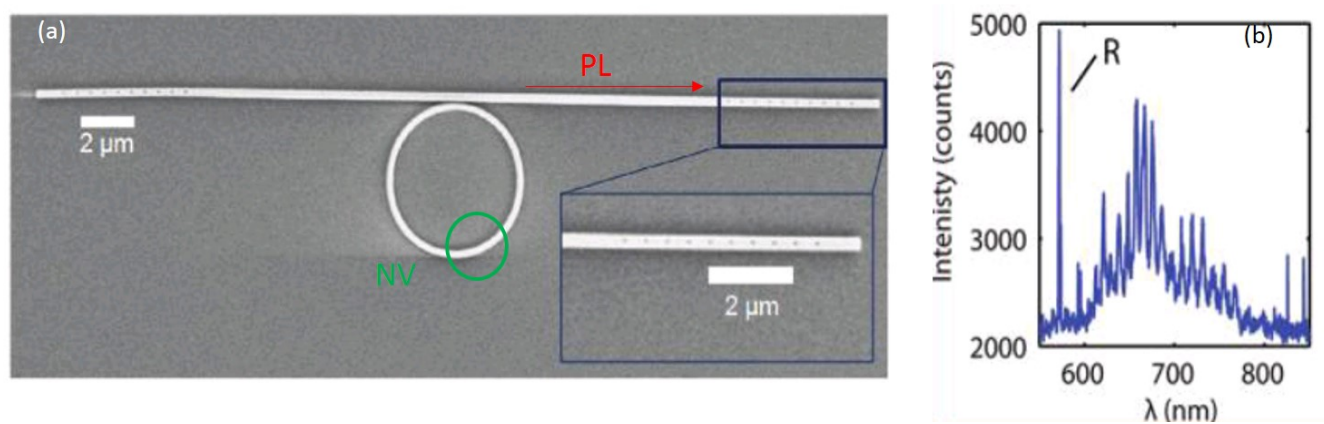

Figure 27: Outcoupling from microring resonators via a ridge waveguide (a) SEM image of the microring and waveguide integrated architecture. Grating couplers are located at the each end (detailed inset). Schematic of NV location and outcoupled collection indicates with the room temperature spectrum shown in (b). The NV spectrum is overlaid with the microring resonances. The Raman line is indicated by R. Reprinted and adapted with permission from [103]. Copyright 2012 American Chemical Society. 


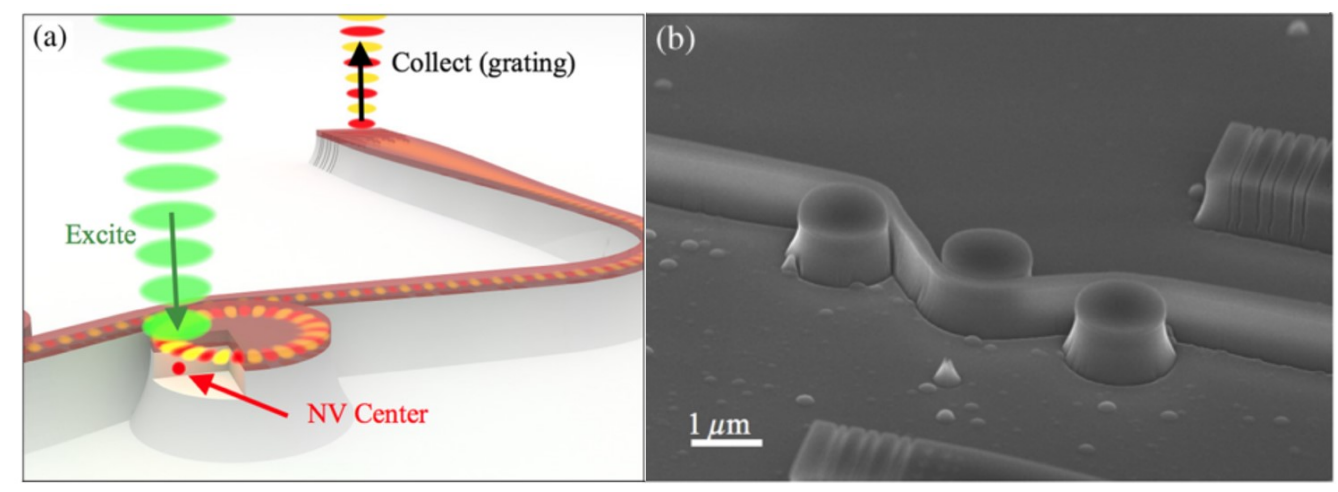

Figure 28: GaP-on-diamond integrated architecture. (a) Schematic diagram of GaP (orange) waveguide coupled disk resonator on diamond (white). $\mathrm{NV}$ located in proximity to the $\mathrm{GaP}$ microdisk is excited as shown, with waveguide coupled fluorescence collected from the grating (b) SEM image of the system. Reprinted with permission from [199]. Copyright 2016 by the American Physical Society.

band-gap of $E_{g}=2.26 \mathrm{eV}$ and high refractive index $n=3.3$, a GaP layer bonded to a diamond photonic structure [194], shown schematically in figure 28a, can act as an efficient waveguide, whilst being transparent to $\mathrm{NV}$ emission. In addition $\mathrm{GaP}$ also displays electro-optical properties and may enable in-situ tuning, switching and routing in an integrated device [197, 198]. Further advantages lie in the availability of uniform thickness, large area wafers, circumventing one of the outstanding challenges of integrated all-diamond approaches (section 3). For colour centre coupling however, the emitter and mode maxima are situated in different materials, reducing the coupling strength. Early hybrid structures demonstrated with NV emission coupled to GaP ridge waveguides at cryogenic temperatures [192] and room-temperature coupling to $Q>2.5 \times 10^{4}$ $\mathrm{GaP}$ microdisks [193]. Later $\mathrm{Fu}$ and coworkers investigated $\mathrm{GaP}$ microrings and microdisks on diamond, demonstrating low temperature coupling to NV ensembles, with photons extracted through tapered fiber coupling. Only a modest change in the PL lifetime was observed, attributed to ensemble averaging and spatial mismatch [196].

Further work by Thomas et al [197] demonstrated the coupling of diamond microdisks to integrated ridge waveguides and their use to out-couple fluorescence from NV centre ensembles. Grating couplers were used to extract the fluorescence at either end of the waveguide. Loaded Qfactors are measured up to $Q_{t o t} \simeq 3800$, combined with mode volumes of $V_{\text {mode }} \sim 3.5\left(\frac{\lambda}{n}\right)^{3}\left(F_{P} \simeq\right.$ $80)$. Recent work by Gould et al $[198,199]$ demonstrated waveguide-coupled disk resonators, directional couplers and grating couplers, all based on the hybrid GaP-diamond system, shown in figure 28a,b. In this work low temperature coupling to single NV centres was also reported with device mode volumes similar to previously fabricated devices with $V_{\text {mode }} \sim 3.5\left(\frac{\lambda}{n}\right)^{3}$ [197], and improved Q-factors of $Q \simeq 8200$, such that an optimised $F_{P}=180$ is expected. It is noted that with the NV centres situated around $20 \mathrm{~nm}$ away from the GaP interface, the measured lifetimes of $8.7 \mathrm{~ns}$ are shorter than those in bulk diamond even in the absence of a cavity resonance, due to the higher local optical density of states created by the nearby GaP. On-resonance with the microdisk modes, the lifetime reduces to $4.7 \mathrm{~ns}$, corresponding to $F_{c a v}=26$. The fluorescence was out-coupled through the waveguide mode with an efficiency of $\eta_{c o}=0.09$. 


\subsection{Open Cavities}

The open cavity has recently emerged as a viable photonic interface for a host of emitters in the solid state $[206,223]$ including colour centres in diamond. These Fabry-Pérot-type resonators, most commonly in a planar-concave configuration of opposing, highly reflective mirrors as in figure 29(d), offer an attractive alternative to integrated diamond based photonic approaches. By decoupling the resonator geometry from the diamond structure, the challenges of precision formation of structures in diamond are considerably mitigated, and both the lateral spatial positioning and the emitter-cavity resonance can be tuned in-situ. The geometry supports modes that comprise confined Gaussian beams (and their transverse harmonics) such that emission couples efficiently to external optics.

To a first approximation the Q-factor of an open cavity scales with the mirror separation, such that the cavity finesse $\mathcal{F}$, determined by the round trip absorption, scattering and transmission losses, remains constant. The Purcell factor of an empty cavity is related to the geometry [224] by:

$$
F_{P}=\frac{6 \lambda}{\pi} \frac{\mathcal{F}}{\sqrt{2 R L-L^{2}}}
$$

where $L$ is the cavity length (mirror separation plus a field penetration depth of $\sim \lambda$ into either mirror), and $R$ is the radius of curvature for the concave mirror.

\subsubsection{Fabrication of Open Cavities}

Dielectric mirrors deposited using the ion beam sputtering technique on super-polished planar and large radius-of-curvature substrates can achieve losses down to a few parts-per-million, bringing $\mathcal{F} \sim 10^{6}$. For micro-mirrors the principal challenge is the fabrication of a smooth concave surface of the required radius of curvature $R$, so that additional scattering losses can be avoided. Methods for doing so have previously involved microsphere templates, bubble cleaving, wet etching and transfer printing [225, 226, 227, 228]. More recently $\mathrm{CO}_{2}$ laser ablation $[229,224,230,231]$ and FIB milling $[232,233]$, have become prominent. Of these methods $\mathrm{CO}_{2}$ laser ablation has been popular due to the ultra-smooth finish it can produce on a concave silica surface as a result of the melting (known also as 'reflowing') of a thin surface layer. A cavity finesse of $\mathcal{F}=1.7 \times 10^{5}$ has been achieved [234] which, if combined with realistic dimensions $R=10 \mu \mathrm{m}$ and $L=2 \mu \mathrm{m}$, could provide for Purcell factors as large as $F_{P} \simeq 50,000$.

Despite the $\mathrm{CO}_{2}$ laser wavelength of $10 \mu \mathrm{m}$, radii of curvature as small as $5 \mu \mathrm{m}$ have been produced, although the nature of the ablation process is such that combining these small $R$ with cavity lengths short enough for stable cavity modes is challenging and requires additional processing steps [231]. FIB-milling can also achieve sub-nanometre roughness due to an intrinsic polishing effect [235], and so far cavities with $\mathcal{F} \simeq 40,000$ have been achieved with this method. Minimum ion beam diameters below $10 \mathrm{~nm}$ combined with precise control of dwell time and flexible patterning software allow nanometre precision over the mirror shape to be achieved [233]. Such flexibility and control offers considerable scope for the further optimisation and engineering of open cavity systems.

Out-coupling of photons from open cavities is generally through one of the mirrors, which is coated with fewer Bragg pairs to provide slightly lower reflectivity. Concave mirrors have been 

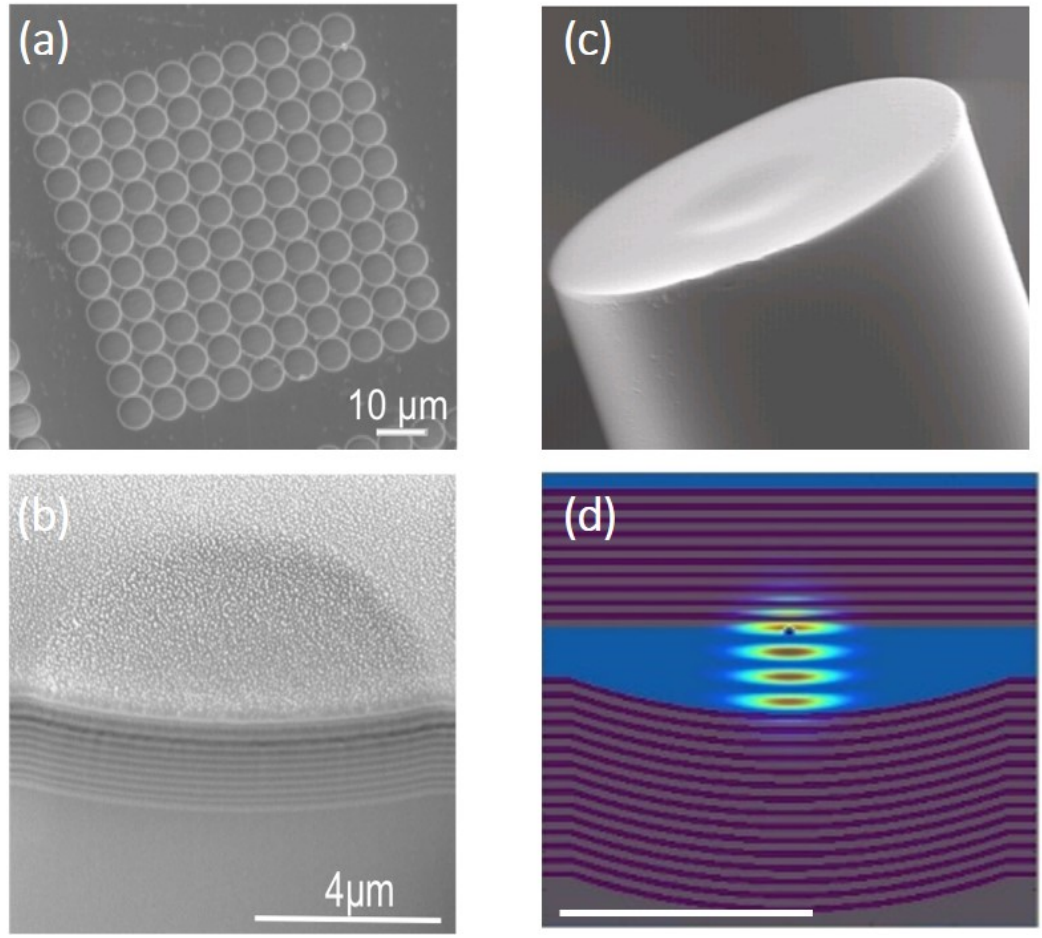

Figure 29: Open Cavity fabrication. (a) SEM image of an array of concave features milled via the FIB method. Reproduced with permission from [232]. (b) A cross-sectional SEM image of a single concave mirror with radius of curvaature $R=10 \mu \mathrm{m}$. The alternating layers of high and low index dielectric layers are apparent and retain the geometry of the underlying structure. (c) A concave feature fabricated onto a fiber end facet with $\mathrm{CO}_{2}$ laser machining. Reproduced with permission from [224]. (d) Finite difference time domain simulation of the electric field intensity in the open-cavity geometry. The high and low index layers of the DBR are indicated in purple and grey respectively. There are four antinodes of the electric field between the surfaces in this instance, whilst penetration of the mode into the mirror substrates can also be observed. Reproduced with permission from [236]. 
produced in large arrays on silica substrates (figure 29(a)) for coupling to free-space optics, and on cleaved optical fiber facets as in figure 29(c). This latter approach has been popularised in recent years by members of the atom optics community where they are known as 'fibre cavities', and may in the future allow direct integration with a large-scale fiber network. It is important to note however that the relatively low numerical aperture of optical fibres provides a limiting factor to the mode volume of fibre cavities that can achieve high out-coupling efficiency $\eta_{c o}$.

\subsubsection{Colour Centre Coupling to Open Cavities}

There have now been several proof of principle demonstrations of colour centre coupling to the open cavity system. These have primarily been conducted at room temperature with both ensembles and single colour centres in nanodiamond [237, 238, 239, 240, 241]. For applications in quantum networks, it is required that these operate in a cryogenic environment for the enhancement of coherent ZPL emission. Towards this goal, Johnson and coworkers demonstrated the tunable coupling to the ZPL transitions (fig. 30a) of a single NV centre in nanodiamond at 77K [236]. FIB milled cavities were used with a radius of curvature of $7.6 \mu \mathrm{m}$, to accomplish mode volumes down to $4.7 \frac{\lambda}{n}^{3}$ and $F_{P} \simeq 50$. A comparison of the in-cavity and free space emission spectrum of the same $\mathrm{NV}$ centre at $77 \mathrm{~K}$ is shown in figure $30 \mathrm{~b}$. The enhanced intensity of the ZPL transitions can be observed, whilst the broad PSB emission no-longer couples to the external optics for the in-cavity emission. The enhancement is quantified by a $40 \%$ increase in the decay rate $\left(F_{c a v} \simeq 10\right)$, and providing a factor 6.25 enhancement in the measured ZPL intensity (figure 30c). This is shown to have an explicit mode-volume dependence, demonstrated by coupling a series of subsequent $\mathrm{TEM}_{00}$ modes to the ZPL as the cavity length is tuned. Single photon emission into the cavity mode is shown in figure $30 \mathrm{~d}$.

These coupling experiments have so far been exclusively carried out using colour centres in nanodiamond to facilitate their inclusion between the mirror substrates, with separations up to a few microns. Whilst electron spin-coherence times for NV-defects in CVD nanodiamonds have been demonstrated to approach those seen in bulk [242, 243, 244], there has been only one report of optical transitions in nanodiamond approaching the transform limit [245]. Therefore the use of single crystal diamond membranes is an important direction for applications in quantum networks. Janitz and coworkers presented a demonstration of such a system through an investigation into the effects of including a diamond membrane of thickness $>10 \mu \mathrm{m}$ within a high finesse fibre based open cavity [246], shown in figure 31a. Crucially, the incorporation of the membrane did not significantly compromise the finesse of the system, measured through transmission experiments in figures $31 \mathrm{~b}, \mathrm{c}$, with $\mathcal{F}$ still in excess of $10^{4}$. A more recent experiment demonstrated the cryogenic implementation of a membrane-cavity in a closed-cycle cryostat, maintaining $\mathcal{F}$ $>10^{4}$ with only passive vibration isolation [247].

Successful implementations to date of cavity enhanced zero-phonon lines for both the NV and $\mathrm{SiV}$ in diamond are summarised in table 1 and figure 32. 

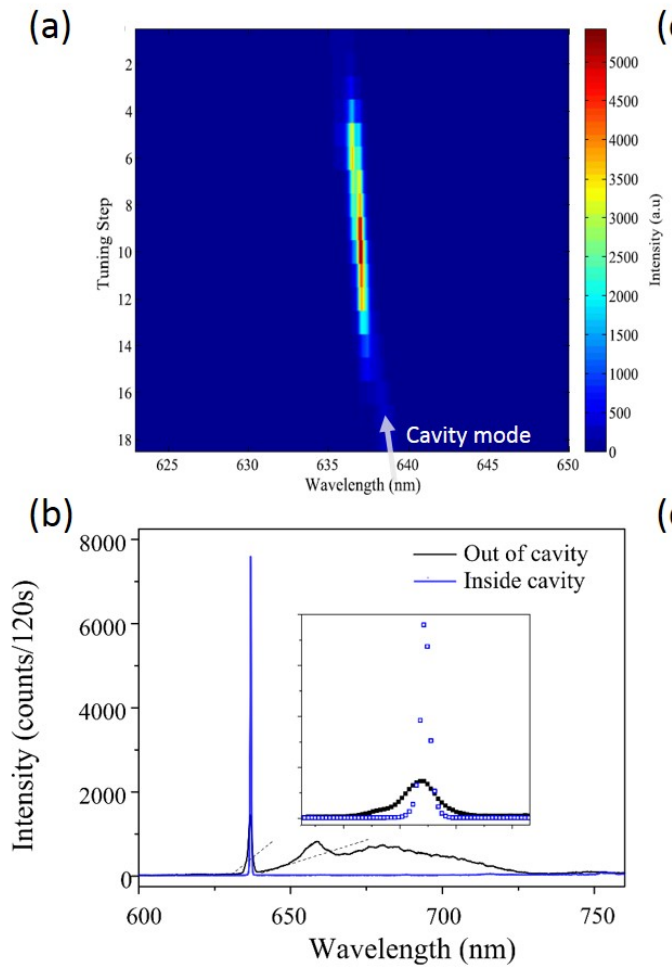

(c)

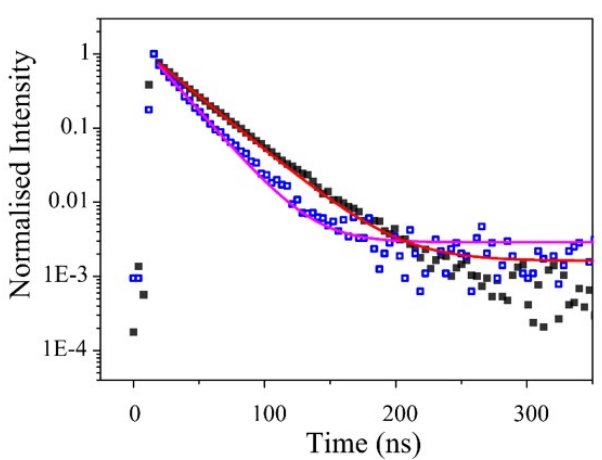

(d)(i)

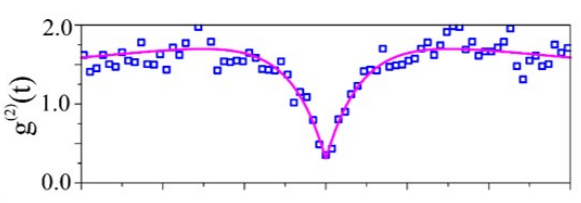

(ii)

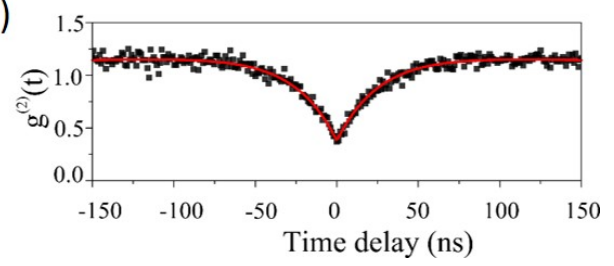

Figure 30: Tunable coupling to the zero-phonon line of a single NV in nanodiamond at 77K. (a) 2D spectral plot showing tuning of the cavity mode (indicated by the white arrow) across the strain-split doublet of a single NV. (b-d) A direct comparison of in-cavity (blue) and on-substrate (black) emission can be observed by studying the emitter with and without the hemispherical substrate. (b) PL spectrum (c) Lifetime measurements at $V_{\text {mode }}=4.7 \frac{\lambda}{n}^{3}$ corresponding to a $40 \%$ reduction in lifetime. (d) Photon intensity correlation measurements showing single photon emission from (i) the cavity coupled zero-phonon line and (ii) out of cavity spectrum. Reproduced with permission from [236].
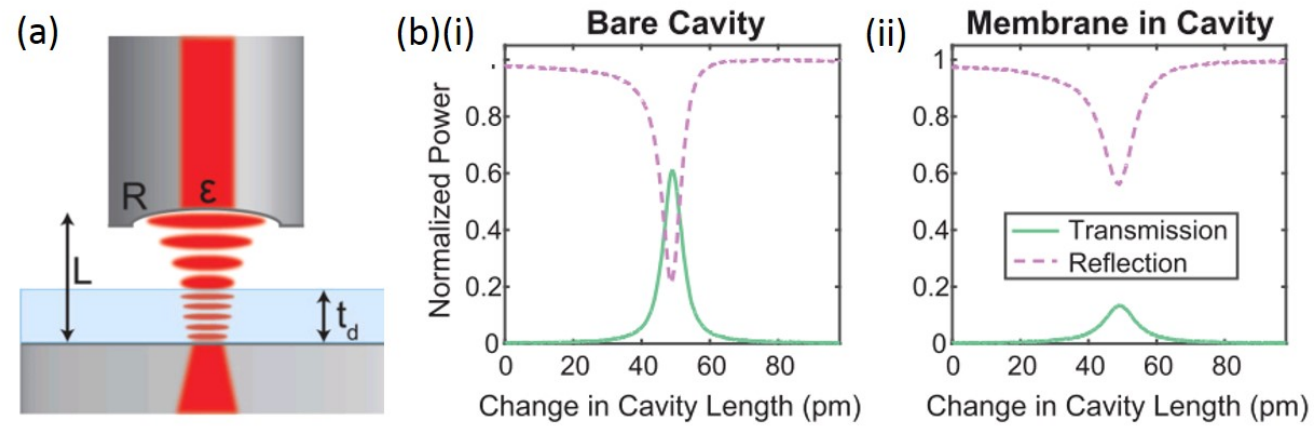

Figure 31: Diamond membrane in an open cavity.(a) Schematic diagram: measured transmission (solid) and reflection (dashed) for the (b) bare cavity and and (c) membrane-in-cavity case, as a function of cavity length. There is a slight reduction in the finesse upon the inclusion of the membrane, reducing the Q-factor at this length. Reprinted with permission from [246]. Copyright 2015 by the American Physical Society. 
Table 1: Cavity enhanced zero-phonon lines in diamond ( *nanodiamond)

\begin{tabular}{|c|c|c|c|c|c|}
\hline Cavity & Colour Centre & $Q$ & $V\left(\frac{1}{n}\right)^{3}$ & $F_{\text {cav }}($ measured $)$ & Author \\
\hline \multicolumn{6}{|c|}{ Photonic Crystal Cavities } \\
\hline 2D PCC & NV & 3000 & 0.88 & 70 & Faraon et al 2012. [51] \\
\hline 2D PCC & $\operatorname{SiV}(300 K)$ & 430 & 1.7 & 19 & Riedrich-Möller et al 2014. [71] \\
\hline 2D PCC & SiV ensemble $(300 \mathrm{~K})$ & 400 & 1.5 & 2.8 & Riedrich-Möller et al 2012. [70] \\
\hline Nanobeam & $\mathrm{NV}$ & 3300 & 1.05 & 62 & Li et al. $2015[52]$ \\
\hline Nanobeam & NV ensemble & 7000 & 0.47 & 26 & Lee et al. 2014 [97] \\
\hline Nanobeam & NV & 1630 & 3.7 & 7 & Hausmann et al. 2013 [200] \\
\hline \multicolumn{6}{|c|}{ Whispering Gallery Resonators } \\
\hline Microring & NV & 5500 & 15 & 12 & Faraon et al. 2013 [190] \\
\hline Microring & $\mathrm{NV}$ & 4300 & $17-32$ & 12 & Faraon et al. 2011 [102] \\
\hline Microdisk & SiV ensemble $(300 \mathrm{~K})$ & 2200 & 9.6 & 1.3 & Lee at al. 2012 [69] \\
\hline \multicolumn{6}{|c|}{ Hybrid Resonators } \\
\hline GaP microdisk & NV & 8200 & 3.5 & 26 & Gould et al. 2016 [199] \\
\hline \multicolumn{6}{|c|}{ Open Cavities } \\
\hline FIB cavity & NV* & 3000 & 4.7 & 10 & Johnson et al. [236] \\
\hline
\end{tabular}

Cavity enhanced zero-phonon lines in diamond

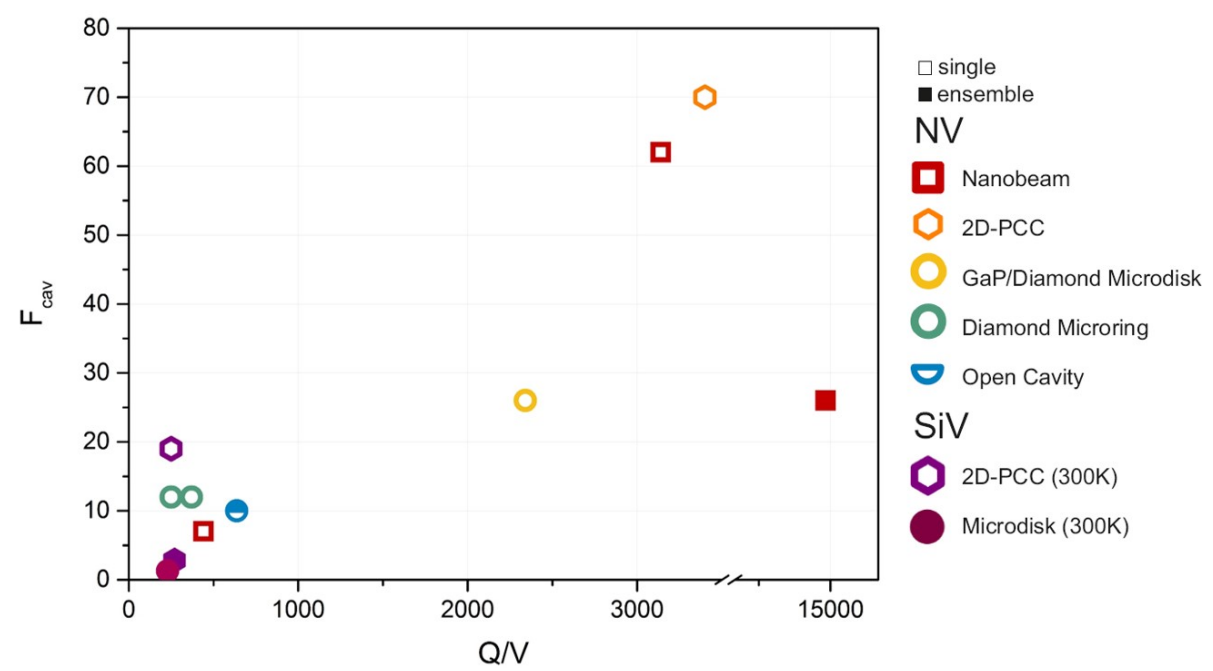

Figure 32: State of the art cavity enhanced ZPL measurements in diamond, summarising table 1. The measured Purcell enhancement is plotted as a function of the $\mathrm{Q} / \mathrm{V}\left(\frac{\lambda}{n}\right)^{-3}$ figure of merit for comparison between resonator geometries. All measurements are at cryogenic temperatures unless otherwise stated. The open shapes represent single colour centre coupling, whilst the filled shapes show ensembles. Reference [97] achieves only a modest $F_{\text {cav }}$, despite a high Q/V metric. This is attributed to a mismatch of the narrow cavity mode to the broader ensemble of ZPL transition frequencies. 


\section{Conclusions and Future Outlook}

Colour centres in diamond have in recent years revealed considerable potential for use as nodes in quantum networks. The NV centre has been well established as one of the pre-eminent solid state qubit systems, and pioneering experiments have demonstrated the first building blocks of a quantum network in which entanglement between stationary NV nodes is generated through quantum interference of spontaneously emitted photons. The NV displays remarkable coherence of the ground state electron spin, particularly with the application of dynamical-decoupling radio-frequency control sequences to alleviate decoherence from the spin-bath, and the advent of isotopically pure ${ }^{12} \mathrm{C}$ diamond materials. The optical properties are one of the major impediments to further scaling up of NV networks, with only $\approx 3 \%$ of the emission in the ZPL severely limiting the entanglement generation rate. The $\mathrm{SiV}$ has emerged as a second candidate qubit, with spin-dependent optical transitions and around $70 \%$ of the emission originating the ZPL. The electron spin of $\mathrm{SiV}$ can be manipulated using picosecond laser pulses, but its coherence time is currently much shorter at $\sim 100 \mathrm{~ns}$. Work is ongoing to extend this coherence, but the degree to which this can be achieved is for now an open question.

The application of these colour centres in a quantum network will require an efficient photonic interface between the node and the network channels for the communication of optically coherent, indistinguishable photons. This is a particularly demanding requirement for the NV, in which the ZPL transition energies are highly sensitive to external electric fields and strain in the diamond lattice. The requirement of indistinguishability between nodes has necessitated external electrostatic tuning to ensure resonance and additional optical control fields to sustain the negative charge state under resonant excitation. In this respect the $\mathrm{SiV}$ displays much better properties. Its split-vacancy configuration possesses inversion symmetry which renders the $\mathrm{SiV}$ transitions largely unperturbed by fluctuating electric fields. This brings two benefits: that there is a large degree of uniformity between defects which may eliminate the requirement for the tuning of defects into resonance, and improved robustness to integration into photonic structures.

Non-resonant photonic structures have provided a good preliminary strategy to improve the collection of indistinguishable photons, and the solid-immersion lens in particular has facilitated the first demonstrations of a NV-based quantum network. In pursuit of larger-scale networks, resonant structures offer greater enhancement rates for indistinguishable photons to increase the entanglement generation rate. Several architectures are being explored towards this goal, the most impressive colour centre coupling results so far having originated from the photonic crystal cavity structures, namely the 2D-PCC and nanobeam structures. Ultimately, monolithic all-diamond resonators offer a route towards fully scalable chip-based devices, but substantial challenges remain regarding the yield with which such devices can be fabricated and the need to tune each cavity independently to the desired resonance. Hybrid approaches with piezoelectric materials such as GaP may offer a route forwards in this regard. With recent demonstrations of diamond integrated superconducting single-photon detection [177], one can start to envisage that a fully integrated device architecture may become a reality.

In the nearer term, newly emerging open cavities provide the distinct advantage of in-situ alignment and tuning, and relaxed requirements for the positioning of colour centres within optical microstructures. The retention of highly coherent electron spins and optical transitions for colour centres positioned in diamond microstructures is nevertheless an ongoing challenge, with no one method providing a complete solution as yet. Rapid progress continues to be made in 
several areas however, with new and refined techniques for diamond etching, control of surface chemistry, colour centre implantation, and lattice damage removal appearing on a regular basis. At this rate of progress it appears likely that quantum networks of colour centres containing multiple nodes may be realised in the next few years.

\section{Acknowledgments}

The authors acknowledge support from the United Kingdom Engineering and Physical Sciences Research Council (EPSRC) through the Networked Quantum Information Technologies (NQIT) hub (grant EP/M013243/1), and the European Union Seventh Framework Programme (FP7/2007-2013) through the Wavelength Tunable Advanced Single Photon Sources (WASPS) programme (grant agreement 618078). 
[1] R. Raussendorf, H. J. Briegel, A One-Way Quantum Computer, Physical Review Letters 86 (22) (2001) 51885191. doi:10.1103/PhysRevLett.86.5188.

[2] H. J. Kimble, The quantum internet, Nature 453 (7198) (2008) 1023-1030. arXiv:0806.4195, doi:10.1038/nature07127.

[3] I. Bloch, Quantum coherence and entanglement with ultracold atoms in optical lattices, Nature 453 (7198) (2008) 1016-1022. doi:10.1038/nature07126.

[4] S. Benjamin, B. Lovett, J. Smith, Prospects for measurement-based quantum computing with solid state spins, Laser \& Photonics Review 3 (6) (2009) 556-574. doi:10.1002/lpor.200810051.

[5] G. Balasubramanian, P. Neumann, D. Twitchen, M. Markham, R. Kolesov, N. Mizuochi, J. Isoya, J. Achard, J. Beck, J. Tissler, V. Jacques, P. R. Hemmer, F. Jelezko, J. Wrachtrup, Ultralong spin coherence time in isotopically engineered diamond, Nature Materials 8 (5) (2009) 383-387. doi:10.1038/nmat2420.

[6] N. Bar-Gill, L. Pham, A. Jarmola, D. Budker, R. Walsworth, Solid-state electronic spin coherence time approaching one second, Nature Communications 4 (2013) 1743. doi:10.1038/ncomms2771.

[7] P. C. Maurer, G. Kucsko, C. Latta, L. Jiang, N. Y. Yao, S. D. Bennett, F. Pastawski, D. Hunger, N. Chisholm, M. Markham, D. J. Twitchen, J. I. Cirac, M. D. Lukin, Room-Temperature Quantum Bit Memory Exceeding One Second, Science 336 (6086) (2012) 1283-1286. doi:10.1126/science.1220513.

[8] S. Yang, Y. Wang, D. D. B. Rao, T. Hien Tran, A. S. Momenzadeh, M. Markham, D. J. Twitchen, P. Wang, W. Yang, R. Stöhr, P. Neumann, H. Kosaka, J. Wrachtrup, High-fidelity transfer and storage of photon states in a single nuclear spin, Nature Photonics 10 (8) (2016) 507-511. arXiv:1511.04939, doi:10.1038/nphoton.2016.103.

[9] L. Robledo, L. Childress, H. Bernien, B. Hensen, P. F. a. Alkemade, R. Hanson, High-fidelity projective read-out of a solid-state spin quantum register, Nature 477 (7366) (2011) 574-578. arXiv:1301.0392v1, doi:10.1038/nature10401.

[10] D. P. DiVincenzo, The Physical Implementation of Quantum Computation, Fortschritte der Physik 48 (9-11) (2000) 771-783.

[11] H. Bernien, B. Hensen, W. Pfaff, G. Koolstra, M. S. Blok, L. Robledo, T. H. Taminiau, M. Markham, D. J. Twitchen, L. Childress, R. Hanson, Heralded entanglement between solid-state qubits separated by three metres, Nature 497 (7447) (2013) 86-90. doi:10.1038/nature12016.

[12] W. Pfaff, B. J. Hensen, H. Bernien, S. B. van Dam, M. S. Blok, T. H. Taminiau, M. J. Tiggelman, R. N. Schouten, M. Markham, D. J. Twitchen, R. Hanson, Unconditional quantum teleportation between distant solid-state quantum bits, Science 345 (6196) (2014) 532-535. doi:10.1126/science.1253512.

[13] B. Hensen, H. Bernien, A. E. Dréau, A. Reiserer, N. Kalb, M. S. Blok, J. Ruitenberg, R. F. L. Vermeulen, R. N. Schouten, C. Abellán, W. Amaya, V. Pruneri, M. W. Mitchell, M. Markham, D. J. Twitchen, D. Elkouss, S. Wehner, T. H. Taminiau, R. Hanson, Loophole-free Bell inequality violation using electron spins separated by 1.3 kilometres, Nature 526 (7575) (2015) 682-686. doi:10.1038/nature15759.

[14] C. Cabrillo, J. I. Cirac, P. García-Fernández, P. Zoller, Creation of entangled states of distant atoms by interference, Physical Review A 59 (2) (1999) 1025-1033. arXiv:9810013, doi:10.1103/PhysRevA.59.1025.

[15] S. D. Barrett, P. Kok, Efficient high-fidelity quantum computation using matter qubits and linear optics, Physical Review A 71 (6) (2005) 060310. arXiv:0408040, doi:10.1103/PhysRevA.71.060310.

[16] X.-L. Feng, Z.-M. Zhang, X.-D. Li, S.-Q. Gong, Z.-Z. Xu, Entangling Distant Atoms by Interference of Polarized Photons, Physical Review Letters 90 (21) (2003) 217902. doi:10.1103/PhysRevLett.90.217902.

[17] Y. L. Lim, A. Beige, L. C. Kwek, Repeat-Until-Success Linear Optics Distributed Quantum Computing, Physical Review Letters 95 (3) (2005) 030505. arXiv:0408043, doi:10.1103/PhysRevLett.95.030505.

[18] S. C. Benjamin, J. Eisert, T. M. Stace, Optical generation of matter qubit graph states, New Journal of Physics 7 (2005) 194-194. arXiv:0506110, doi:10.1088/1367-2630/7/1/194.

[19] C. Y. Hu, A. Young, J. L. OBrien, W. J. Munro, J. G. Rarity, Giant optical Faraday rotation induced by a singleelectron spin in a quantum dot: Applications to entangling remote spins via a single photon, Physical Review B 78 (8) (2008) 085307. arXiv:0708.2019, doi:10.1103/PhysRevB.78.085307.

[20] K. Nemoto, M. Trupke, S. J. Devitt, A. M. Stephens, B. Scharfenberger, K. Buczak, T. Nöbauer, M. S. Everitt, J. Schmiedmayer, W. J. Munro, Photonic Architecture for Scalable Quantum Information Processing in Diamond, Physical Review X 4 (3) (2014) 031022. arXiv:1309.4277, doi:10.1103/PhysRevX.4.031022.

[21] T. Grange, G. Hornecker, D. Hunger, J.-p. Poizat, J.-m. Gérard, P. Senellart, A. Auffèves, Cavity-Funneled Generation of Indistinguishable Single Photons from Strongly Dissipative Quantum Emitters, Physical Review Letters 114 (19) (2015) 193601. doi:10.1103/PhysRevLett.114.193601.

URL http://link.aps.org/doi/10.1103/PhysRevLett.114.193601

[22] S. C. Benjamin, D. E. Browne, J. Fitzsimons, J. J. L. Morton, Brokered graph-state quantum computation, New Journal of Physics 8 (8) (2006) 141-141. arXiv:0509209, doi:10.1088/1367-2630/8/8/141.

[23] A. Reiserer, N. Kalb, M. S. Blok, K. J. M. van Bemmelen, T. H. Taminiau, R. Hanson, D. J. Twitchen, M. Markham, Robust Quantum-Network Memory Using Decoherence-Protected Subspaces of Nuclear Spins, Physical Review X 6 (2) (2016) 021040. doi:10.1103/PhysRevX.6.021040. 
[24] N. H. Nickerson, J. F. Fitzsimons, S. C. Benjamin, Freely Scalable Quantum Technologies Using Cells of 5-to-50 Qubits with Very Lossy and Noisy Photonic Links, Physical Review X 4 (4) (2014) 041041. arXiv:1406.0880, doi:10.1103/PhysRevX.4.041041.

[25] F. Dolde, V. Bergholm, Y. Wang, I. Jakobi, B. Naydenov, S. Pezzagna, J. Meijer, F. Jelezko, P. Neumann, T. Schulte-Herbrüggen, J. Biamonte, J. Wrachtrup, High-fidelity spin entanglement using optimal control, Nature Communications 5 (2014) 3371. arXiv:1309.4430, doi:10.1038/ncomms4371.

[26] G. Waldherr, Y. Wang, S. Zaiser, M. Jamali, T. Schulte-Herbrüggen, H. Abe, T. Ohshima, J. Isoya, J. F. Du, P. Neumann, J. Wrachtrup, Quantum error correction in a solid-state hybrid spin register, Nature 506 (7487) (2014) 204-207. arXiv:arXiv:1309.6424v1, doi:10.1038/nature12919.

[27] T. H. Taminiau, J. Cramer, T. van der Sar, V. V. Dobrovitski, R. Hanson, Universal control and error correction in multi-qubit spin registers in diamond, Nature Nanotechnology 9 (3) (2014) 171-176. arXiv:arXiv:1309.5452v2, doi:10.1038/nnano.2014.2.

[28] J. Cramer, N. Kalb, M. A. Rol, B. Hensen, M. S. Blok, M. Markham, D. J. Twitchen, R. Hanson, T. H. Taminiau, Repeated quantum error correction on a continuously encoded qubit by real-time feedback, Nature Communications 7 (11526) (2016) 11526. arXiv:1508.01388, doi:10.1038/ncomms11526.

[29] A. Zaitsev, Optical Properties of Diamond: A Data Handbook, 1st Edition, Springer, 2001.

[30] I. Aharonovich, E. Neu, Diamond Nanophotonics, Advanced Optical Materials 2 (10) (2014) 911-928. doi:10.1002/adom.201400189.

[31] E. Togan, Y. Chu, a. S. Trifonov, L. Jiang, J. Maze, L. Childress, M. V. G. Dutt, a. S. Sø rensen, P. R. Hemmer, a. S. Zibrov, M. D. Lukin, Quantum entanglement between an optical photon and a solid-state spin qubit, Nature 466 (7307) (2010) 730-734. doi:10.1038/nature09256.

[32] L. Rondin, J.-P. Tetienne, T. Hingant, J.-F. Roch, P. Maletinsky, V. Jacques, Magnetometry with nitrogen-vacancy defects in diamond, Reports on Progress in Physics 77 (5) (2014) 056503. arXiv:1311.5214, doi:10.1088/0034 4885/77/5/056503.

[33] D. Le Sage, K. Arai, D. R. Glenn, S. J. DeVience, L. M. Pham, L. Rahn-Lee, M. D. Lukin, A. Yacoby, A. Komeili, R. L. Walsworth, Optical magnetic imaging of living cells, Nature 496 (7446) (2013) 486-489. doi:10.1038/nature 12072 .

[34] A. Gruber, A. Drabenstedt, C. Tietz, L. Fleurt, J. Wrachtrup, C. von Borczyskowski, Scanning Confocal Optical Microscopy and Magnetic Resonance on Single Defect Centers, Science 276 (5321) (1997) 2012-2014. doi:10.1126/science.276.5321.2012.

[35] C. Kurtsiefer, S. Mayer, P. Zarda, H. Weinfurter, Stable Solid-State Source of Single Photons, Physical Review Letters 85 (2) (2000) 290-293. doi:10.1103/PhysRevLett.85.290.

[36] F. Jelezko, T. Gaebel, I. Popa, A. Gruber, J. Wrachtrup, Observation of Coherent Oscillations in a Single Electron Spin, Physical Review Letters 92 (7) (2004) 076401. doi:10.1103/PhysRevLett.92.076401.

[37] F. Jelezko, T. Gaebel, I. Popa, M. Domhan, A. Gruber, J. Wrachtrup, Observation of Coherent Oscillation of a Single Nuclear Spin and Realization of a Two-Qubit Conditional Quantum Gate, Physical Review Letters 93 (13) (2004) 130501. doi:10.1103/PhysRevLett.93.130501.

[38] L. Childress, M. V. Gurudev Dutt, J. M. Taylor, a. S. Zibrov, F. Jelezko, J. Wrachtrup, P. R. Hemmer, M. D. Lukin, Coherent Dynamics of Coupled Electron and Nuclear Spin Qubits in Diamond, Science 314 (5797) (2006) 281-285. doi:10.1126/science.1131871.

[39] M. W. Doherty, N. B. Manson, P. Delaney, F. Jelezko, J. Wrachtrup, L. C. Hollenberg, The nitrogen-vacancy colour centre in diamond, Physics Reports 528 (1) (2013) 1-45. arXiv:1302.3288, doi:10.1016/j.physrep.2013.02.001.

[40] G. de Lange, Z. H. Wang, D. Riste, V. V. Dobrovitski, R. Hanson, Universal Dynamical Decoupling of a Single Solid-State Spin from a Spin Bath, Science 330 (6000) (2010) 60-63. arXiv:1008.2119, doi:10.1126/science.1192739.

[41] C. a. Ryan, J. S. Hodges, D. G. Cory, Robust Decoupling Techniques to Extend Quantum Coherence in Diamond, Physical Review Letters 105 (20) (2010) 200402. arXiv:1008.2197, doi:10.1103/PhysRevLett.105.200402.

[42] B. Naydenov, F. Dolde, L. T. Hall, C. Shin, H. Fedder, L. C. L. Hollenberg, F. Jelezko, J. Wrachtrup, Dynamical decoupling of a single-electron spin at room temperature, Physical Review B 83 (8) (2011) 081201. arXiv:1008.1953, doi:10.1103/PhysRevB.83.081201

[43] M. V. G. Dutt, L. Childress, L. Jiang, E. Togan, J. Maze, F. Jelezko, a. S. Zibrov, P. R. Hemmer, M. D. Lukin, Quantum Register Based on Individual Electronic and Nuclear Spin Qubits in Diamond, Science 316 (5829) (2007) 1312-1316. doi:10.1126/science.1139831.

[44] P. Neumann, J. Beck, M. Steiner, F. Rempp, H. Fedder, P. R. Hemmer, J. Wrachtrup, F. Jelezko, Single-Shot Readout of a Single Nuclear Spin, Science 329 (5991) (2010) 542-544. doi:10.1126/science.1189075.

[45] A. Dréau, P. Spinicelli, J. R. Maze, J.-F. Roch, V. Jacques, Single-Shot Readout of Multiple Nuclear Spin Qubits in Diamond under Ambient Conditions, Physical Review Letters 110 (6) (2013) 060502. arXiv:1210.6195v2, doi:10.1103/PhysRevLett.110.060502. 
URL http://link.aps.org/doi/10.1103/PhysRevLett.110.060502

[46] N. Bar-Gill, L. Pham, C. Belthangady, D. Le Sage, P. Cappellaro, J. Maze, M. Lukin, A. Yacoby, R. Walsworth, Suppression of spin-bath dynamics for improved coherence of multi-spin-qubit systems, Nature Communications 3 (May) (2012) 858. arXiv:arXiv:1112.0667v2, doi:10.1038/ncomms1856.

[47] A. Batalov, V. Jacques, F. Kaiser, P. Siyushev, P. Neumann, L. J. Rogers, R. L. McMurtrie, N. B. Manson, F. Jelezko, J. Wrachtrup, Low Temperature Studies of the Excited-State Structure of Negatively Charged NitrogenVacancy Color Centers in Diamond, Physical Review Letters 102 (19) (2009) 195506. arXiv:arXiv:0906.3426v1, doi:10.1103/PhysRevLett.102.195506.

[48] K.-M. C. Fu, C. Santori, P. E. Barclay, L. J. Rogers, N. B. Manson, R. G. Beausoleil, Observation of the Dynamic Jahn-Teller Effect in the Excited States of Nitrogen-Vacancy Centers in Diamond, Physical Review Letters 103 (25) (2009) 256404. doi:10.1103/PhysRevLett.103.256404.

[49] T. a. Abtew, Y. Y. Sun, B.-C. Shih, P. Dev, S. B. Zhang, P. Zhang, Dynamic Jahn-Teller Effect in the NV Center in Diamond, Physical Review Letters 107 (14) (2011) 146403. doi:10.1103/PhysRevLett.107.146403.

[50] P. Tamarat, T. Gaebel, J. R. Rabeau, M. Khan, a. D. Greentree, H. Wilson, L. C. L. Hollenberg, S. Prawer, P. Hemmer, F. Jelezko, J. Wrachtrup, Stark Shift Control of Single Optical Centers in Diamond, Physical Review Letters 97 (8) (2006) 083002. arXiv:0607170, doi:10.1103/PhysRevLett.97.083002.

[51] A. Faraon, C. Santori, Z. Huang, V. M. Acosta, R. G. Beausoleil, Coupling of Nitrogen-Vacancy Centers to Photonic Crystal Cavities in Monocrystalline Diamond, Physical Review Letters 109 (3) (2012) 033604 doi:10.1103/PhysRevLett.109.033604.

[52] L. Li, T. Schröder, E. H. Chen, M. Walsh, I. Bayn, J. Goldstein, O. Gaathon, M. E. Trusheim, M. Lu, J. Mower, M. Cotlet, M. L. Markham, D. J. Twitchen, D. Englund, Coherent spin control of a nanocavity-enhanced qubit in diamond, Nature Communications 6 (2015) 6173. doi:10.1038/ncomms7173.

[53] K.-M. C. Fu, C. Santori, P. E. Barclay, R. G. Beausoleil, Conversion of neutral nitrogen-vacancy centers to negatively charged nitrogen-vacancy centers through selective oxidation, Applied Physics Letters 96 (12) (2010) 121907. arXiv:1001.5449, doi:10.1063/1.3364135

URL http://scitation.aip.org/content/aip/journal/apl/96/12/10.1063/1.3364135

[54] T. Gaebel, M. Domhan, C. Wittmann, I. Popa, F. Jelezko, J. Rabeau, A. Greentree, S. Prawer, E. Trajkov, P. Hemmer, J. Wrachtrup, Photochromism in single nitrogen-vacancy defect in diamond, Applied Physics B 82 (2) (2006) 243-246. doi:10.1007/s00340-005-2056-2.

[55] P. Siyushev, H. Pinto, M. Vörös, A. Gali, F. Jelezko, J. Wrachtrup, Optically Controlled Switching of the Charge State of a Single Nitrogen-Vacancy Center in Diamond at Cryogenic Temperatures, Physical Review Letters 110 (16) (2013) 167402. doi:10.1103/PhysRevLett.110.167402.

[56] V. M. Acosta, C. Santori, A. Faraon, Z. Huang, K.-M. C. Fu, A. Stacey, D. a. Simpson, K. Ganesan, S. Tomljenovic-Hanic, a. D. Greentree, S. Prawer, R. G. Beausoleil, Dynamic Stabilization of the Optical Resonances of Single Nitrogen-Vacancy Centers in Diamond, Physical Review Letters 108 (20) (2012) 206401. arXiv:1112.5490, doi:10.1103/PhysRevLett.108.206401

[57] Y. Chu, N. de Leon, B. Shields, B. Hausmann, R. Evans, E. Togan, M. J. Burek, M. Markham, A. Stacey, A. Zibrov, A. Yacoby, D. Twitchen, M. Loncar, H. Park, P. Maletinsky, M. Lukin, Coherent Optical Transitions in Implanted Nitrogen Vacancy Centers, Nano Letters 14 (4) (2014) 1982-1986. doi:10.1021/n1404836p. URL http://pubs .acs .org/doi/abs/10.1021/nl404836p

[58] H. F. Fotso, A. E. Feiguin, D. D. Awschalom, V. V. Dobrovitski, Suppressing Spectral Diffusion of Emitted Photons with Optical Pulses, Physical Review Letters 116 (3) (2016) 033603. arXiv:1512.05754, doi:10.1103/PhysRevLett.116.033603.

[59] H. Bernien, L. Childress, L. Robledo, M. Markham, D. Twitchen, R. Hanson, Two-Photon Quantum Interference from Separate Nitrogen Vacancy Centers in Diamond, Physical Review Letters 108 (4) (2012) 043604 arXiv:arXiv:1110.3329v1, doi:10.1103/PhysRevLett.108.043604.

[60] A. Sipahigil, M. L. Goldman, E. Togan, Y. Chu, M. Markham, D. J. Twitchen, a. S. Zibrov, A. Kubanek, M. D. Lukin, Quantum Interference of Single Photons from Remote Nitrogen-Vacancy Centers in Diamond, Physical Review Letters 108 (14) (2012) 143601. arXiv:1112.3975, doi:10.1103/PhysRevLett.108.143601. URL http://link.aps .org/doi/10.1103/PhysRevLett.108.143601

[61] C. K. Hong, Z. Y. Ou, L. Mandel, Measurement of Subpicosecond Time Intervals between Two Photons by Interference, Physical Review Letters 59 (18) (1987) 523-523. doi:10.1103/PhysRevLett.58.523.

[62] D. L. Moehring, P. Maunz, S. Olmschenk, K. C. Younge, D. N. Matsukevich, L.-M. Duan, C. Monroe, Entanglement of single-atom quantum bits at a distance, Nature 449 (7158) (2007) 68-71. doi:10.1038/nature06118. URL http://www . nature.com/doifinder/10.1038/nature06118

[63] T. Müller, C. Hepp, B. Pingault, E. Neu, S. Gsell, M. Schreck, H. Sternschulte, D. Steinmüller-Nethl, C. Becher, M. Atatüre, Optical signatures of silicon-vacancy spins in diamond, Nature Communications 5 (2014) 3328 arXiv:1312.2997, doi:10.1038/ncomms4328.

[64] C. Hepp, T. Müller, V. Waselowski, J. N. Becker, B. Pingault, H. Sternschulte, D. Steinmüller-Nethl, A. Gali, J. R. 
Maze, M. Atatüre, C. Becher, Electronic Structure of the Silicon Vacancy Color Center in Diamond, Physical Review Letters 112 (3) (2014) 036405. arXiv:1310.3106, doi:10.1103/PhysRevLett.112.036405.

[65] L. J. Rogers, K. D. Jahnke, M. W. Doherty, A. Dietrich, L. P. McGuinness, C. Müller, T. Teraji, H. Sumiya, J. Isoya, N. B. Manson, F. Jelezko, Electronic structure of the negatively charged silicon-vacancy center in diamond, Physical Review B 89 (23) (2014) 235101. arXiv:arXiv:1310.3131v2, doi:10.1103/PhysRevB.89.235101. URL http://link.aps.org/doi/10.1103/PhysRevB.89.235101

[66] L. Rogers, K. Jahnke, T. Teraji, L. Marseglia, C. Müller, B. Naydenov, H. Schauffert, C. Kranz, J. Isoya, L. McGuinness, F. Jelezko, Multiple intrinsically identical single-photon emitters in the solid state, Nature Communications 5 (2014) 4739. doi:10.1038/ncomms5739.

[67] E. Neu, D. Steinmetz, J. Riedrich-Möller, S. Gsell, M. Fischer, M. Schreck, C. Becher, Single photon emission from silicon-vacancy colour centres in chemical vapour deposition nano-diamonds on iridium, New Journal of Physics 13 (2) (2011) 025012. doi:10.1088/1367-2630/13/2/025012.

[68] E. Neu, M. Agio, C. Becher, Photophysics of single silicon vacancy centers in diamond: implications for single photon emission, Optics Express 20 (18) (2012) 19956. doi:10.1364/OE.20.019956.

[69] J. C. Lee, I. Aharonovich, A. P. Magyar, F. Rol, E. L. Hu, Coupling of silicon-vacancy centers to a single crystal diamond cavity, Optics Express 20 (8) (2012) 8891. doi:10.1364/OE.20.008891.

[70] J. Riedrich-Möller, L. Kipfstuhl, C. Hepp, E. Neu, C. Pauly, F. Mücklich, A. Baur, M. Wandt, S. Wolff, M. Fischer, S. Gsell, M. Schreck, C. Becher, One- and two-dimensional photonic crystal microcavities in single crystal diamond, Nature Nanotechnology 7 (1) (2012) 69-74. doi:10.1038/nnano.2011.190.

[71] J. Riedrich-Möller, C. Arend, C. Pauly, F. Mücklich, M. Fischer, S. Gsell, M. Schreck, C. Becher, Deterministic Coupling of a Single Silicon-Vacancy Color Center to a Photonic Crystal Cavity in Diamond, Nano Letters 14 (9) (2014) 5281-5287. doi:10.1021/n1502327b.

[72] J. Riedrich-Möller, S. Pezzagna, J. Meijer, C. Pauly, F. Mücklich, M. Markham, A. M. Edmonds, C. Becher, Nanoimplantation and Purcell enhancement of single nitrogen-vacancy centers in photonic crystal cavities in diamond, Applied Physics Letters 106 (22) (2015) 221103. arXiv:1503.05666, doi:10.1063/1.4922117. URL http://scitation.aip.org/content/aip/journal/apl/106/22/10.1063/1.4922117

[73] E. Neu, R. Albrecht, M. Fischer, S. Gsell, M. Schreck, C. Becher, Electronic transitions of single silicon vacancy centers in the near-infrared spectral region, Physical Review B 85 (24) (2012) 245207. arXiv:1204.4994v1, doi:10.1103/PhysRevB.85.245207.

[74] A. Sipahigil, K. D. Jahnke, L. J. Rogers, T. Teraji, J. Isoya, A. S. Zibrov, F. Jelezko, M. D. Lukin, Indistinguishable Photons from Separated Silicon-Vacancy Centers in Diamond, Physical Review Letters 113 (11) (2014) 113602. arXiv:1406.4268, doi:10.1103/PhysRevLett.113.113602.

[75] J. L. Zhang, H. Ishiwata, T. M. Babinec, M. Radulaski, K. Müller, K. G. Lagoudakis, C. Dory, J. Dahl, R. Edgington, V. Soulière, G. Ferro, A. a. Fokin, P. R. Schreiner, Z.-X. Shen, N. a. Melosh, J. Vučković, Hybrid Group IV Nanophotonic Structures Incorporating Diamond Silicon-Vacancy Color Centers, Nano Letters 16 (1) (2016) 212-217. arXiv:1509.01617, doi:10.1021/acs.nanolett.5b03515.

[76] K. D. Jahnke, A. Sipahigil, J. M. Binder, M. W. Doherty, M. Metsch, L. J. Rogers, N. B. Manson, M. D. Lukin, F. Jelezko, Electronphonon processes of the silicon-vacancy centre in diamond, New Journal of Physics 17 (4) (2015) 043011. doi:10.1088/1367-2630/17/4/043011.

[77] J. N. Becker, J. Görlitz, C. Arend, M. Markham, C. Becher, Ultrafast all-optical coherent control of single silicon vacancy colour centres in diamond, Nature Communications 7 (2016) 13512. arXiv:1603.00789, doi:10.1038/ncomms13512.

[78] B. Pingault, J. N. Becker, C. H. H. Schulte, C. Arend, C. Hepp, T. Godde, A. I. Tartakovskii, M. Markham, C. Becher, M. Atatüre, All-Optical Formation of Coherent Dark States of Silicon-Vacancy Spins in Diamond, Physical Review Letters 113 (26) (2014) 263601. doi:10.1103/PhysRevLett.113.263601.

[79] L. J. Rogers, K. D. Jahnke, M. H. Metsch, A. Sipahigil, J. M. Binder, T. Teraji, H. Sumiya, J. Isoya, M. D. Lukin, P. Hemmer, F. Jelezko, All-Optical Initialization, Readout, and Coherent Preparation of Single Silicon-Vacancy Spins in Diamond, Physical Review Letters 113 (26) (2014) 263602. doi:10.1103/PhysRevLett.113.263602.

[80] A. Sipahigil, K. D. Jahnke, L. J. Rogers, T. Teraji, J. Isoya, A. S. Zibrov, F. Jelezko, M. D. Lukin, Indistinguishable Photons from Separated Silicon-Vacancy Centers in Diamond (Supplementary Information), Physical Review Letters 113 (11) (2014) 113602. arXiv:1406.4268, doi:10.1103/PhysRevLett.113.113602. URL http://link.aps.org/doi/10.1103/PhysRevLett.113.113602

[81] I. Aharonovich, S. Castelletto, D. a. Simpson, C.-H. Su, a. D. Greentree, S. Prawer, Diamond-based single-photon emitters, Reports on Progress in Physics 74 (7) (2011) 076501. doi:10.1088/0034-4885/74/7/076501.

[82] J. M. Smith, F. Grazioso, B. R. Patton, P. R. Dolan, M. L. Markham, D. J. Twitchen, Optical properties of a single-colour centre in diamond with a green zero-phonon line, New Journal of Physics 13 (4) (2011) 045005 doi:10.1088/1367-2630/13/4/045005.

[83] S.-Y. Lee, M. Widmann, T. Rendler, M. W. Doherty, T. M. Babinec, S. Yang, M. Eyer, P. Siyushev, B. J. M. Hausmann, M. Loncar, Z. Bodrog, A. Gali, N. B. Manson, H. Fedder, J. Wrachtrup, Readout and control of a single nu- 
clear spin with a metastable electron spin ancilla, Nature Nanotechnology 8 (7) (2013) 487-492. arXiv:1302.4608, doi:10.1038/nnano.2013.104.

[84] T. Iwasaki, F. Ishibashi, Y. Miyamoto, Y. Doi, S. Kobayashi, T. Miyazaki, K. Tahara, K. D. Jahnke, L. J. Rogers, B. Naydenov, F. Jelezko, S. Yamasaki, S. Nagamachi, T. Inubushi, N. Mizuochi, M. Hatano, Germanium-Vacancy Single Color Centers in Diamond, Scientific Reports 5 (2015) 12882. doi:10.1038/srep12882.

[85] P. Siyushev, M. H. Metsch, A. Ijaz, J. M. Binder, M. K. Bhaskar, D. D. Sukachev, A. Sipahigil, R. E. Evans, C. T. Nguyen, M. D. Lukin, P. R. Hemmer, Y. N. Palyanov, I. N. Kupriyanov, Y. M. Borzdov, L. J. Rogers, F. Jelezko, Optical and microwave control of germanium-vacancy center spins in diamond (2016) 1-6arXiv:1612.02947. URL http: //arxiv.org/abs/1612.02947v1

[86] M. K. Bhaskar, D. D. Sukachev, A. Sipahigil, R. E. Evans, M. J. Burek, C. T. Nguyen, L. J. Rogers, P. Siyushev, M. H. Metsch, H. Park, F. Jelezko, M. Lončar, M. D. Lukin, Quantum Nonlinear Optics with a GermaniumVacancy Color Center in a Nanoscale Diamond Waveguide (2016) 1-5arXiv:1612.03036.

[87] M. J. Burek, Y. Chu, M. S. Z. Liddy, P. Patel, J. Rochman, S. Meesala, W. Hong, Q. Quan, M. D. Lukin, M. Lončar, High quality-factor optical nanocavities in bulk single-crystal diamond, Nature Communications 5 (2014) 5718. arXiv: 1408.5973, doi:10.1038/ncomms6718.

[88] Y. Tao, C. Degen, Facile Fabrication of Single-Crystal-Diamond Nanostructures with Ultrahigh Aspect Ratio, Advanced Materials 25 (29) (2013) 3962-3967. doi:10.1002/adma.201301343.

[89] P. Rath, S. Ummethala, C. Nebel, W. H. P. Pernice, Diamond as a material for monolithically integrated optical and optomechanical devices, physica status solidi (a) 212 (11) (2015) 2385-2399. doi:10.1002/pssa.201532494.

[90] B. J. M. Hausmann, I. Bulu, V. Venkataraman, P. Deotare, M. Lončar, Diamond nonlinear photonics, Nature Photonics 8 (5) (2014) 369-374. doi:10.1038/nphoton.2014.72.

[91] A. H. Piracha, P. Rath, K. Ganesan, S. Kühn, W. H. P. Pernice, S. Prawer, Scalable Fabrication of Integrated Nanophotonic Circuits on Arrays of Thin Single Crystal Diamond Membrane Windows, Nano Letters 16 (5) (2016) 3341-3347. doi:10.1021/acs.nanolett.6b00974.

[92] P. Olivero, S. Rubanov, P. Reichart, B. C. Gibson, S. T. Huntington, J. Rabeau, A. D. Greentree, J. Salzman, D. Moore, D. N. Jamieson, S. Prawer, Ion-Beam-Assisted Lift-Off Technique for Three-Dimensional Micromachining of Freestanding Single-Crystal Diamond, Advanced Materials 17 (20) (2005) 2427-2430. doi:10.1002/adma.200500752.

[93] B. a. Fairchild, P. Olivero, S. Rubanov, A. D. Greentree, F. Waldermann, R. a. Taylor, I. Walmsley, J. M. Smith, S. Huntington, B. C. Gibson, D. N. Jamieson, S. Prawer, Fabrication of Ultrathin Single-Crystal Diamond Membranes, Advanced Materials 20 (24) (2008) 4793-4798. arXiv:arXiv:0809.2133, doi:10.1002/adma.200801460.

[94] B. R. Patton, P. R. Dolan, F. Grazioso, M. B. Wincott, J. M. Smith, M. L. Markham, D. J. Twitchen, Y. Zhang, E. Gu, M. D. Dawson, B. a. Fairchild, A. D. Greentree, S. Prawer, Optical properties of single crystal diamond microfilms fabricated by ion implantation and lift-off processing, Diamond and Related Materials 21 (2012) 1623. doi:10.1016/j.diamond.2011.09.006.

[95] A. P. Magyar, J. C. Lee, A. M. Limarga, I. Aharonovich, F. Rol, D. R. Clarke, M. Huang, E. L. Hu, Fabrication of thin, luminescent, single-crystal diamond membranes, Applied Physics Letters 99 (8) (2011) 081913. arXiv:1108.0738, doi:10.1063/1.3628463.

[96] I. Aharonovich, J. C. Lee, A. P. Magyar, B. B. Buckley, C. G. Yale, D. D. Awschalom, E. L. Hu, Homoepitaxial Growth of Single Crystal Diamond Membranes for Quantum Information Processing, Advanced Materials 24 (10) (2012) OP54-OP59. arXiv:1109.5634, doi:10.1002/adma.201103932.

[97] J. C. Lee, D. O. Bracher, S. Cui, K. Ohno, C. a. McLellan, X. Zhang, P. Andrich, B. Alemán, K. J. Russell, A. P. Magyar, I. Aharonovich, A. Bleszynski Jayich, D. Awschalom, E. L. Hu, Deterministic coupling of delta-doped nitrogen vacancy centers to a nanobeam photonic crystal cavity, Applied Physics Letters 105 (26) (2014) 261101. arXiv:1411.0725, doi:10.1063/1.4904909.

[98] I. Bayn, B. Meyler, J. Salzman, R. Kalish, Triangular nanobeam photonic cavities in single-crystal diamond, New Journal of Physics 13 (2) (2011) 025018. doi:10.1088/1367-2630/13/2/025018.

[99] L. Li, T. Schröder, E. H. Chen, H. Bakhru, D. Englund, One-dimensional photonic crystal cavities in single-crystal diamond, Photonics and Nanostructures - Fundamentals and Applications 15 (2015) $130-136$. doi:10.1016/j.photonics.2015.03.002.

[100] T. Schröder, S. L. Mouradian, J. Zheng, M. E. Trusheim, M. Walsh, E. H. Chen, L. Li, I. Bayn, D. Englund, Quantum nanophotonics in diamond [Invited], Journal of the Optical Society of America B 33 (4) (2016) B65. arXiv:1603.05339, doi:10.1364/JOSAB.33.000B65.

[101] R. P. Mildren, J. R. Rabeau, Optical Engineering of Diamond, Wiley-VCH Verlag GmbH \& Co. KGaA, Weinheim, Germany, 2013. doi:10.1002/9783527648603. URL http://doi.wiley.com/10.1002/9783527648603

[102] A. Faraon, P. E. Barclay, C. Santori, K.-M. C. Fu, R. G. Beausoleil, Resonant enhancement of the zerophonon emission from a colour centre in a diamond cavity, Nature Photonics 5 (5) (2011) 301-305. doi:10.1038/nphoton.2011.52. 
[103] B. J. M. Hausmann, B. Shields, Q. Quan, P. Maletinsky, M. McCutcheon, J. T. Choy, T. M. Babinec, A. Kubanek, A. Yacoby, M. D. Lukin, M. Lončar, Integrated diamond networks for quantum nanophotonics, Nano Letters 12 (3) (2012) 1578-1582. arXiv:1111.5330v1, doi:10.1021/nl204449n

[104] M. J. Burek, N. P. de Leon, B. J. Shields, B. J. M. Hausmann, Y. Chu, Q. Quan, A. S. Zibrov, H. Park, M. D. Lukin, M. Lončar, Free-Standing Mechanical and Photonic Nanostructures in Single-Crystal Diamond, Nano Letters 12 (12) (2012) 6084-6089. doi:10.1021/n1302541e.

[105] H. W. Choi, E. Gu, C. Liu, C. Griffin, J. M. Girkin, I. M. Watson, M. D. Dawson, Fabrication of natural diamond microlenses by plasma etching, Journal of Vacuum Science \& Technology B: Microelectronics and Nanometer Structures 23 (1) (2005) 130. doi:10.1116/1.1843826.

[106] C. Lee, H. Choi, E. Gu, M. Dawson, H. Murphy, Fabrication and characterization of diamond micro-optics, Diamond and Related Materials 15 (4-8) (2006) 725-728. doi:10.1016/j.diamond.2005.09.033.

[107] C. Lee, E. Gu, M. Dawson, Micro-cylindrical and micro-ring lenses in CVD diamond, Diamond and Related Materials 16 (4-7) (2007) 944-948. doi:10.1016/j.diamond.2006.11.027.

[108] C. Lee, E. Gu, M. Dawson, I. Friel, G. Scarsbrook, Etching and micro-optics fabrication in diamond using chlorine-based inductively-coupled plasma, Diamond and Related Materials 17 (7-10) (2008) 1292-1296. doi:10.1016/j.diamond.2008.01.011.

[109] P. Appel, E. Neu, M. Ganzhorn, A. Barfuss, M. Batzer, M. Gratz, A. Tschöpe, P. Maletinsky, Fabrication of all diamond scanning probes for nanoscale magnetometry, Review of Scientific Instruments 87 (6) (2016) 063703 arXiv:1604.00021, doi:10.1063/1.4952953.

[110] S. Cui, Near-surface Nitrogen Vacancy Centers in Diamond, Ph.D. thesis, Harvard University (2014).

[111] Y. Tao, J. M. Boss, B. a. Moores, C. L. Degen, Single-crystal diamond nanomechanical resonators with quality factors exceeding one million, Nature Communications 5 (2014) 3638. arXiv:1212.1347, doi:10.1038/ncomms4638.

[112] A. K. Tiwari, J. P. Goss, P. R. Briddon, N. G. Wright, A. B. Horsfall, R. Jones, H. Pinto, M. J. Rayson, Calculated electron affinity and stability of halogen-terminated diamond, Physical Review B 84 (24) (2011) 245305 doi:10.1103/PhysRevB.84.245305.

[113] D. Riedel, D. Rohner, M. Ganzhorn, T. Kaldewey, P. Appel, E. Neu, R. J. Warburton, P. Maletinsky, Low-Loss Broadband Antenna for Efficient Photon Collection from a Coherent Spin in Diamond, Physical Review Applied 2 (6) (2014) 064011. arXiv:1408.4117, doi:10.1103/PhysRevApplied.2.064011.

URL http://link.aps .org/doi/10.1103/PhysRevApplied.2.064011

[114] P. Maletinsky, S. Hong, M. S. Grinolds, B. Hausmann, M. D. Lukin, R. L. Walsworth, M. Loncar, A. Yacoby, A robust scanning diamond sensor for nanoscale imaging with single nitrogen-vacancy centres, Nature Nanotechnology 7 (5) (2012) 320-324. doi:10.1038/nnano.2012.50.

[115] L. Li, T. Schröder, E. H. Chen, H. Bakhru, D. Englund, One-dimensional photonic crystal cavities in single-crystal diamond, Photonics and Nanostructures - Fundamentals and Applications 15 (2015) 130-136. doi:10.1016/j.photonics.2015.03.002.

URL http://dx.doi.org/10.1016/j.photonics.2015.03.002

[116] M. Kim, H. J. Mamin, M. H. Sherwood, C. T. Rettner, J. Frommer, D. Rugar, Effect of oxygen plasma and thermal oxidation on shallow nitrogen-vacancy centers in diamond, Applied Physics Letters 105 (4) (2014) 042406. doi:10.1063/1.4891839.

[117] A. Denisenko, A. Romanyuk, C. Pietzka, J. Scharpf, E. Kohn, Surface damages in diamond by Ar/O[sub 2] plasma and their effect on the electrical and electrochemical characteristics of boron-doped layers, Journal of Applied Physics 108 (7) (2010) 074901. doi:10.1063/1.3489986.

[118] S. Cui, A. S. Greenspon, K. Ohno, B. a. Myers, A. C. B. Jayich, D. D. Awschalom, E. L. Hu, Reduced PlasmaInduced Damage to Near-Surface Nitrogen-Vacancy Centers in Diamond, Nano Letters 15 (5) (2015) 2887-2891. doi:10.1021/acs.nanolett.5b00457.

[119] F. Fávaro de Oliveira, S. A. Momenzadeh, Y. Wang, M. Konuma, M. Markham, A. M. Edmonds, A. Denisenko, J. Wrachtrup, Effect of low-damage inductively coupled plasma on shallow nitrogen-vacancy centers in diamond, Applied Physics Letters 107 (7) (2015) 073107. arXiv:1507.00890, doi:10.1063/1.4929356.

[120] J. P. Hadden, J. P. Harrison, a. C. Stanley-Clarke, L. Marseglia, Y.-L. D. Ho, B. R. Patton, J. L. OBrien, J. G. Rarity, Strongly enhanced photon collection from diamond defect centers under microfabricated integrated solid immersion lenses, Applied Physics Letters 97 (24) (2010) 241901. doi:10.1063/1.3519847. URL http://scitation.aip.org/content/aip/journal/apl/97/24/10.1063/1.3519847

[121] S. Castelletto, J. P. Harrison, L. Marseglia, a. C. Stanley-Clarke, B. C. Gibson, B. a. Fairchild, J. P. Hadden, Y.L. D. Ho, M. P. Hiscocks, K. Ganesan, S. T. Huntington, F. Ladouceur, a. D. Greentree, S. Prawer, J. L. O'Brien, J. G. Rarity, Diamond-based structures to collect and guide light, New Journal of Physics 13 (2) (2011) 025020. doi:10.1088/1367-2630/13/2/025020.

[122] L. Marseglia, J. P. Hadden, a. C. Stanley-Clarke, J. P. Harrison, B. Patton, Y.-L. D. Ho, B. Naydenov, F. Jelezko, J. Meijer, P. R. Dolan, J. M. Smith, J. G. Rarity, J. L. OBrien, Nanofabricated solid immersion lenses registered to single emitters in diamond, Applied Physics Letters 98 (13) (2011) 133107. doi:10.1063/1.3573870. 
[123] T. M. Babinec, J. T. Choy, K. J. M. Smith, M. Khan, M. Loncar, Design and focused ion beam fabrication of single crystal diamond nanobeam cavities, Journal of Vacuum Science \& Technology B: Microelectronics and Nanometer Structures 29 (1) (2011) 010601. doi:10.1116/1.3520638.

[124] I. Bayn, B. Meyler, A. Lahav, J. Salzman, R. Kalish, B. a. Fairchild, S. Prawer, M. Barth, O. Benson, T. Wolf, P. Siyushev, F. Jelezko, J. Wrachtrup, Processing of photonic crystal nanocavity for quantum information in diamond, Diamond and Related Materials 20 (7) (2011) 937-943. arXiv:1012.5878, doi:10.1016/j.diamond.2011.05.002.

[125] J. O. Orwa, C. Santori, K. M. C. Fu, B. Gibson, D. Simpson, I. Aharonovich, A. Stacey, A. Cimmino, P. Balog, M. Markham, D. Twitchen, A. D. Greentree, R. G. Beausoleil, S. Prawer, Engineering of nitrogen-vacancy color centers in high purity diamond by ion implantation and annealing, Journal of Applied Physics 109 (8) (2011) 083530. doi:10.1063/1.3573768.

[126] J. Orwa, A. Greentree, I. Aharonovich, A. Alves, J. Van Donkelaar, A. Stacey, S. Prawer, Fabrication of single optical centres in diamonda review, Journal of Luminescence 130 (9) (2010) 1646-1654. doi:10.1016/j.jlumin.2009.12.028.

[127] J. Michl, T. Teraji, S. Zaiser, I. Jakobi, G. Waldherr, F. Dolde, P. Neumann, M. W. Doherty, N. B. Manson, J. Isoya, J. Wrachtrup, Perfect alignment and preferential orientation of nitrogen-vacancy centers during chemical vapor deposition diamond growth on (111) surfaces, Applied Physics Letters 104 (10) (2014) 102407. doi:10.1063/1.4868128

[128] M. Lesik, J.-P. Tetienne, A. Tallaire, J. Achard, V. Mille, A. Gicquel, J.-F. Roch, V. Jacques, Perfect preferential orientation of nitrogen-vacancy defects in a synthetic diamond sample, Applied Physics Letters 104 (11) (2014) 113107. doi:10.1063/1.4869103.

[129] E. Neu, P. Appel, M. Ganzhorn, J. Miguel-Sánchez, M. Lesik, V. Mille, V. Jacques, A. Tallaire, J. Achard, P. Maletinsky, Photonic nano-structures on (111)-oriented diamond, Applied Physics Letters 104 (15) (2014) 153108. doi:10.1063/1.4871580.

[130] T. Ishikawa, K.-M. C. Fu, C. Santori, V. M. Acosta, R. G. Beausoleil, H. Watanabe, S. Shikata, K. M. Itoh, Optical and Spin Coherence Properties of Nitrogen-Vacancy Centers Placed in a $100 \mathrm{~nm}$ Thick Isotopically Purified Diamond Layer, Nano Letters 12 (4) (2012) 2083-2087. doi:10.1021/n1300350r.

[131] E. Neu, C. Hepp, M. Hauschild, S. Gsell, M. Fischer, H. Sternschulte, D. Steinmüller-Nethl, M. Schreck, C. Becher, Low-temperature investigations of single silicon vacancy colour centres in diamond, New Journal of Physics 15 (4) (2013) 043005. arXiv:1210.3201, doi:10.1088/1367-2630/15/4/043005.

[132] A. M. Edmonds, M. E. Newton, P. M. Martineau, D. J. Twitchen, S. D. Williams, Electron paramagnetic resonance studies of silicon-related defects in diamond, Physical Review B - Condensed Matter and Materials Physics 77 (24) (2008) 1-11. doi:10.1103/PhysRevB.77.245205.

[133] U. F. S. D’Haenens-Johansson, A. M. Edmonds, B. L. Green, M. E. Newton, G. Davies, P. M. Martineau, R. U. A. Khan, D. J. Twitchen, Optical properties of the neutral silicon split-vacancy center in diamond, Physical Review B 84 (24) (2011) 245208. doi:10.1103/PhysRevB.84.245208.

[134] S. Pezzagna, B. Naydenov, F. Jelezko, J. Wrachtrup, J. Meijer, Creation efficiency of nitrogen-vacancy centres in diamond, New Journal of Physics 12 (6) (2010) 065017. doi:10.1088/1367-2630/12/6/065017.

[135] J. Meijer, B. Burchard, M. Domhan, C. Wittmann, T. Gaebel, I. Popa, F. Jelezko, J. Wrachtrup, Generation of single color centers by focused nitrogen implantation, Applied Physics Letters 87 (26) (2005) 261909. arXiv:0505063, doi:10.1063/1.2103389.

[136] C. Santori, P. E. Barclay, K.-M. C. Fu, R. G. Beausoleil, Vertical distribution of nitrogen-vacancy centers in diamond formed by ion implantation and annealing, Physical Review B 79 (12) (2009) 125313. arXiv:0812.3905, doi:10.1103/PhysRevB.79.125313.

[137] B. Naydenov, F. Reinhard, A. Lammle, V. Richter, R. Kalish, U. F. S. DHaenens-Johansson, M. Newton, F. Jelezko, J. Wrachtrup, Increasing the coherence time of single electron spins in diamond by high temperature annealing, Applied Physics Letters 97 (24) (2010) 242511. doi:10.1063/1.3527975.

[138] C. Wang, C. Kurtsiefer, H. Weinfurter, B. Burchard, Single photon emission from SiV centres in diamond produced by ion implantation, Journal of Physics B: Atomic, Molecular and Optical Physics 39 (1) (2006) 37. doi:10.1088/0953-4075/39/1/005

URL http: //iopscience.iop.org/0953-4075/39/1/005

[139] S. Tamura, G. Koike, A. Komatsubara, T. Teraji, S. Onoda, L. P. McGuinness, L. Rogers, B. Naydenov, E. Wu, L. Yan, F. Jelezko, T. Ohshima, J. Isoya, T. Shinada, T. Tanii, Array of bright silicon-vacancy centers in diamond fabricated by low-energy focused ion beam implantation, Applied Physics Express 7 (11) (2014) 115201. doi:10.7567/APEX.7.115201.

[140] C. Arend, J. N. Becker, H. Sternschulte, D. Steinmüller-Nethl, C. Becher, Photoluminescence excitation and spectral hole burning spectroscopy of silicon vacancy centers in diamond, Physical Review B 94 (4) (2016) 045203. arXiv:1603.04295, doi:10.1103/PhysRevB.94.045203.

[141] R. E. Evans, A. Sipahigil, D. D. Sukachev, A. S. Zibrov, M. D. Lukin, Narrow-Linewidth Homogeneous Optical 
Emitters in Diamond Nanostructures via Silicon Ion Implantation, Physical Review Applied 5 (4) (2016) 044010. arXiv:1512.03820, doi:10.1103/PhysRevApplied.5.044010.

[142] S. Pezzagna, D. Rogalla, D. Wildanger, J. Meijer, A. Zaitsev, Creation and nature of optical centres in diamond for single-photon emissionoverview and critical remarks, New Journal of Physics 13 (3) (2011) 035024. doi:10.1088/1367-2630/13/3/035024.

[143] J. R. Rabeau, P. Reichart, G. Tamanyan, D. N. Jamieson, S. Prawer, F. Jelezko, T. Gaebel, I. Popa, M. Domhan, J. Wrachtrup, Implantation of labelled single nitrogen vacancy centers in diamond using [sup 15]N, Applied Physics Letters 88 (2) (2006) 023113. doi:10.1063/1.2158700.

[144] D. J. Twitchen, M. E. Newton, J. M. Baker, T. R. Anthony, W. F. Banholzer, Electron-paramagnetic-resonance measurements on the divacancy defect center R4/W6 in diamond, Physical Review B 59 (20) (1999) 1290012910. doi:10.1103/PhysRevB.59.12900.

URL http://link.aps.org/doi/10.1103/PhysRevB.59.12900

[145] F. Fávaro de Oliveira, S. A. Momenzadeh, D. Antonov, H. Fedder, A. Denisenko, J. Wrachtrup, On the efficiency of combined ion implantation for the creation of near-surface nitrogen-vacancy centers in diamond, physica status solidi (a) 213 (8) (2016) 2044-2050. doi:10.1002/pssa.201600326.

[146] F. Waldermann, P. Olivero, J. Nunn, K. Surmacz, Z. Wang, D. Jaksch, R. Taylor, I. Walmsley, M. Draganski, P. Reichart, A. Greentree, D. Jamieson, S. Prawer, Creating diamond color centers for quantum optical applications, Diamond and Related Materials 16 (11) (2007) 1887-1895. arXiv:0710.5379, doi:10.1016/j.diamond.2007.09.009.

[147] Z. Huang, W.-D. Li, C. Santori, V. M. Acosta, A. Faraon, T. Ishikawa, W. Wu, D. Winston, R. S. Williams, R. G. Beausoleil, Diamond nitrogen-vacancy centers created by scanning focused helium ion beam and annealing, Applied Physics Letters 103 (8) (2013) 081906. doi:10.1063/1.4819339.

[148] J. F. Ziegler, M. Ziegler, J. Biersack, SRIM The stopping and range of ions in matter (2010), Nuclear Instruments and Methods in Physics Research Section B: Beam Interactions with Materials and Atoms 268 (11-12) (2010) 1818-1823. doi:10.1016/j.nimb.2010.02.091.

[149] M. Schukraft, J. Zheng, T. Schröder, S. L. Mouradian, M. Walsh, M. E. Trusheim, H. Bakhru, D. R. Englund Invited Article: Precision nanoimplantation of nitrogen vacancy centers into diamond photonic crystal cavities and waveguides, APL Photonics 1 (2) (2016) 020801. doi:10.1063/1.4948746.

[150] T. Gaebel, M. Domhan, I. Popa, C. Wittmann, P. Neumann, F. Jelezko, J. R. Rabeau, N. Stavrias, A. D. Greentree, S. Prawer, J. Meijer, J. Twamley, P. R. Hemmer, J. Wrachtrup, Room-temperature coherent coupling of single spins in diamond, Nature Physics 2 (6) (2006) 408-413. doi:10.1038/nphys318.

[151] J. S. Hodges, L. Li, M. Lu, E. H. Chen, M. E. Trusheim, S. Allegri, X. Yao, O. Gaathon, H. Bakhru, D. Englund, Long-lived NV - spin coherence in high-purity diamond membranes, New Journal of Physics 14 doi:10.1088/1367-2630/14/9/093004.

[152] M. Lesik, P. Spinicelli, S. Pezzagna, P. Happel, V. Jacques, O. Salord, B. Rasser, A. Delobbe, P. Sudraud, A. Tallaire, J. Meijer, J.-F. Roch, Maskless and targeted creation of arrays of colour centres in diamond using focused ion beam technology, physica status solidi (a) 210 (10) (2013) 2055-2059. doi:10.1002/pssa.201300102.

[153] S. Pezzagna, D. Wildanger, P. Mazarov, A. D. Wieck, Y. Sarov, I. Rangelow, B. Naydenov, F. Jelezko, S. W. Hell, J. Meijer, Nanoscale Engineering and Optical Addressing of Single Spins in Diamond, Small 6 (19) (2010) 2117-2121. doi:10.1002/smll.201000902.

[154] S. Sangtawesin, T. O. Brundage, Z. J. Atkins, J. R. Petta, Highly-tunable formation of nitrogen-vacancy centers via ion implantation 1 (July) (2014) 3-6. arXiv:arXiv:1407.1434v1.

[155] I. Bayn, E. H. Chen, M. E. Trusheim, L. Li, T. Schröder, O. Gaathon, M. Lu, A. Stein, M. Liu, K. Kisslinger, H. Clevenson, D. Englund, Generation of Ensembles of Individually Resolvable Nitrogen Vacancies Using Nanometer-Scale Apertures in Ultrahigh-Aspect Ratio Planar Implantation Masks, Nano Letters 15 (3) (2015) 1751-1758. arXiv:1412.6600, doi:10.1021/n1504441m.

[156] D. M. Toyli, C. D. Weis, G. D. Fuchs, T. Schenkel, D. D. Awschalom, Chip-Scale Nanofabrication of Single Spins and Spin Arrays in Diamond, Nano Letters 10 (8) (2010) 3168-3172. arXiv:1007.0240, doi:10.1021/nl102066q.

[157] P. Spinicelli, A. Dréau, L. Rondin, F. Silva, J. Achard, S. Xavier, S. Bansropun, T. Debuisschert, S. Pezzagna, J. Meijer, V. Jacques, J.-F. Roch, Engineered arrays of nitrogen-vacancy color centers in diamond based on implantation of CN molecules through nanoapertures, New Journal of Physics 13 (2) (2011) 025014. doi:10.1088/13672630/13/2/025014.

[158] D. Scarabelli, M. Trusheim, O. Gaathon, D. Englund, S. J. Wind, Nanoscale Engineering of Closely-Spaced Electronic Spins in Diamond, Nano Letters 16 (8) (2016) 4982-4990. doi:10.1021/acs.nanolett.6b01692.

[159] S. Pezzagna, D. Rogalla, H.-W. Becker, I. Jakobi, F. Dolde, B. Naydenov, J. Wrachtrup, F. Jelezko, C. Trautmann, J. Meijer, Creation of colour centres in diamond by collimated ion-implantation through nano-channels in mica, physica status solidi (a) 208 (9) (2011) 2017-2022. doi:10.1002/pssa.201100455.

[160] F. Dolde, I. Jakobi, B. Naydenov, N. Zhao, S. Pezzagna, C. Trautmann, J. Meijer, P. Neumann, F. Jelezko, J. Wrachtrup, Room-temperature entanglement between single defect spins in diamond, Nature Physics 9 (3) (2013) 139-143. arXiv:1206.2031, doi:10.1038/nphys2545. 
[161] G. Davies, M. F. Harmer, Optical Studies of the 1.945eV Vibronic Band in Diamond, Proc. R. Soc. Lond. A. 348 (1976) 285-298.

[162] J. Schwartz, S. Aloni, D. F. Ogletree, T. Schenkel, Effects of low-energy electron irradiation on formation of nitrogenvacancy centers in single-crystal diamond, New Journal of Physics 14 (4) (2012) 043024. arXiv:1111.5055, doi:10.1088/1367-2630/14/4/043024.

[163] E. Kim, V. M. Acosta, E. Bauch, D. Budker, P. R. Hemmer, Electron spin resonance shift and linewidth broadening of nitrogen-vacancy centers in diamond as a function of electron irradiation dose, Applied Physics Letters 101 (8) (2012) 082410. doi:10.1063/1.4747211.

[164] Y.-c. Chen, P. S. Salter, S. Knauer, L. Weng, A. C. Frangeskou, C. J. Stephen, S. N. Ishmael, P. R. Dolan, S. Johnson, B. L. Green, G. W. Morley, M. E. Newton, J. G. Rarity, M. J. Booth, J. M. Smith, Laser writing of coherent colour centres in diamond, Nature Photonics 11 (2) (2016) 77-80. arXiv:/arxiv.org/abs/1606.05109, doi:10.1038/nphoton.2016.234.

[165] B. Sun, P. S. Salter, M. J. Booth, High conductivity micro-wires in diamond following arbitrary paths, Applied Physics Letters 105 (23) (2014) 231105. doi:10.1063/1.4902998.

URL http: //scitation.aip.org/content/aip/journal/apl/105/23/10.1063/1.4902998

[166] R. D. Simmonds, P. S. Salter, A. Jesacher, M. J. Booth, Three dimensional laser microfabrication in diamond using a dual adaptive optics system, Optics Express 19 (24) (2011) 24122. doi:10.1364/OE.19.024122.

[167] K. Ohno, F. Joseph Heremans, L. C. Bassett, B. a. Myers, D. M. Toyli, A. C. Bleszynski Jayich, C. J. Palmstrom, D. D. Awschalom, Engineering shallow spins in diamond with nitrogen delta-doping, Applied Physics Letters 101 (8) (2012) 082413. arXiv:1207.2784, doi:10.1063/1.4748280.

[168] K. Ohno, F. Joseph Heremans, C. F. de las Casas, B. a. Myers, B. J. Alemán, A. C. Bleszynski Jayich, D. D. Awschalom, Three-dimensional localization of spins in diamond using 12C implantation, Applied Physics Letters 105 (5) (2014) 052406. arXiv:1405.7352, doi:10.1063/1.4890613.

URL http://dx.doi.org/10.1063/1.4890613 http://scitation.aip.org/content/aip/journal/apl/105/5/10.1063/1.48906

[169] C. a. McLellan, B. a. Myers, S. Kraemer, K. Ohno, D. D. Awschalom, A. C. Bleszynski Jayich, Patterned Formation of Highly Coherent Nitrogen-Vacancy Centers Using a Focused Electron Irradiation Technique, Nano Letters 16 (4) (2016) 2450-2454. arXiv:1512.08821, doi:10.1021/acs.nanolett.5b05304.

URL http://dx.doi.org/10.1021/acs.nanolett.5b05304/nhttp://pubs.acs.org/doi/pdfplus/10.1021/acs.nanolett.5b053 http://pubs.acs.org/doi/abs/10.1021/acs.nanolett.5b05304

[170] B. Grotz, M. V. Hauf, M. Dankerl, B. Naydenov, S. Pezzagna, J. Meijer, F. Jelezko, J. Wrachtrup, M. Stutzmann, F. Reinhard, J. a. Garrido, Charge state manipulation of qubits in diamond, Nature Communications 3 (2012) 729. doi: $10.1038 /$ ncomms 1729.

[171] K. Ohashi, T. Rosskopf, H. Watanabe, M. Loretz, Y. Tao, R. Hauert, S. Tomizawa, T. Ishikawa, J. Ishi-Hayase, S. Shikata, C. L. Degen, K. M. Itoh, Negatively Charged Nitrogen-Vacancy Centers in a $5 \mathrm{~nm}$ Thin 12 C Diamond Film, Nano Letters 13 (10) (2013) 4733-4738. doi:10.1021/nl402286v.

[172] M. Kaviani, P. Deák, B. Aradi, T. Frauenheim, J.-P. Chou, A. Gali, Proper Surface Termination for Luminescent Near-Surface NV Centers in Diamond, Nano Letters 14 (8) (2014) 4772-4777. doi:10.1021/n1501927y.

[173] S. Cui, E. L. Hu, Increased negatively charged nitrogen-vacancy centers in fluorinated diamond, Applied Physics Letters 103 (5) (2013) 051603. doi:10.1063/1.4817651.

[174] C. Osterkamp, J. Scharpf, S. Pezzagna, J. Meijer, T. Diemant, R. Jurgen Behm, B. Naydenov, F. Jelezko, Increasing the creation yield of shallow single defects in diamond by surface plasma treatment, Applied Physics Letters 103 (19) (2013) 193118. doi:10.1063/1.4829875.

[175] L. Li, E. H. Chen, J. Zheng, S. L. Mouradian, F. Dolde, T. Schröder, S. Karaveli, M. L. Markham, D. J. Twitchen, D. Englund, Efficient Photon Collection from a Nitrogen Vacancy Center in a Circular Bullseye Grating, Nano Letters 15 (3) (2015) 1493-1497. arXiv:1409.3068, doi:10.1021/n1503451j.

[176] S. L. Mouradian, T. Schröder, C. B. Poitras, L. Li, J. Goldstein, E. H. Chen, M. Walsh, J. Cardenas, M. L. Markham, D. J. Twitchen, M. Lipson, D. Englund, Scalable Integration of Long-Lived Quantum Memories into a Photonic Circuit, Physical Review X 5 (3) (2015) 031009. arXiv:1409.7965, doi:10.1103/PhysRevX.5.031009.

[177] P. Rath, O. Kahl, S. Ferrari, F. Sproll, G. Lewes-Malandrakis, D. Brink, K. Ilin, M. Siegel, C. Nebel, W. Pernice, Superconducting single-photon detectors integrated with diamond nanophotonic circuits, Light: Science \& Applications 4 (10) (2015) e338. arXiv:1505.04251, doi:10.1038/lsa.2015.111.

[178] P. Siyushev, F. Kaiser, V. Jacques, I. Gerhardt, S. Bischof, H. Fedder, J. Dodson, M. Markham, D. Twitchen, F. Jelezko, J. Wrachtrup, Monolithic diamond optics for single photon detection, Applied Physics Letters 97 (24) (2010) 241902. doi:10.1063/1.3519849.

[179] M. Jamali, I. Gerhardt, M. Rezai, K. Frenner, H. Fedder, J. Wrachtrup, Microscopic diamond solid-immersionlenses fabricated around single defect centers by focused ion beam milling, Review of Scientific Instruments 85 (12) (2014) 123703. arXiv:1406.7209, doi:10.1063/1.4902818.

[180] D. Wildanger, B. R. Patton, H. Schill, L. Marseglia, J. P. Hadden, S. Knauer, A. Schönle, J. G. Rarity, J. L. O’Brien, S. W. Hell, J. M. Smith, Solid Immersion Facilitates Fluorescence Microscopy with Nanome- 
ter Resolution and Sub-Ångström Emitter Localization, Advanced Materials 24 (44) (2012) OP309-OP313. doi:10.1002/adma.201203033.

[181] D. Riedel, D. Rohner, M. Ganzhorn, T. Kaldewey, P. Appel, E. Neu, R. J. Warburton, P. Maletinsky, Low-Loss Broadband Antenna for Efficient Photon Collection from a Coherent Spin in Diamond, Physical Review Applied 2 (6) (2014) 064011. arXiv:1408.4117, doi:10.1103/PhysRevApplied.2.064011.

[182] S. A. Momenzadeh, R. J. Stöhr, F. F. de Oliveira, A. Brunner, A. Denisenko, S. Yang, F. Reinhard, J. Wrachtrup, Nanoengineered Diamond Waveguide as a Robust Bright Platform for Nanomagnetometry Using Shallow Nitrogen Vacancy Centers, Nano Letters 15 (1) (2015) 165-169. arXiv:1409.0027, doi:10.1021/nl503326t.

[183] T. M. Babinec, B. J. M. Hausmann, M. Khan, Y. Zhang, J. R. Maze, P. R. Hemmer, M. Lončar, A diamond nanowire single-photon source, Nature Nanotechnology 5 (3) (2010) 195-199. arXiv:0908.0233, doi:10.1038/nnano.2010.6.

[184] B. J. Hausmann, M. Khan, Y. Zhang, T. M. Babinec, K. Martinick, M. McCutcheon, P. R. Hemmer, M. Lončar, Fabrication of diamond nanowires for quantum information processing applications, Diamond and Related Materials 19 (5-6) (2010) 621-629. arXiv:0908.0352, doi:10.1016/j.diamond.2010.01.011.

[185] B. J. M. Hausmann, T. M. Babinec, J. T. Choy, J. S. Hodges, S. Hong, I. Bulu, A. Yacoby, M. D. Lukin, M. Lončar, Single-color centers implanted in diamond nanostructures, New Journal of Physics 13 (4) (2011) 045004. arXiv:1009.4224, doi:10.1088/1367-2630/13/4/045004

[186] A. Tallaire, J. Achard, A. Boussadi, O. Brinza, A. Gicquel, I. Kupriyanov, Y. Palyanov, G. Sakr, J. Barjon, High quality thick CVD diamond films homoepitaxially grown on (111)-oriented substrates, Diamond and Related Materials 41 (2014) 34-40. doi:10.1016/j.diamond.2013.11.002.

[187] M. S. Grinolds, S. Hong, P. Maletinsky, L. Luan, M. D. Lukin, R. L. Walsworth, A. Yacoby, Nanoscale magnetic imaging of a single electron spin under ambient conditions, Nature Physics 9 (4) (2013) 215-219. doi: $10.1038 /$ nphys 2543

[188] M. P. Hiscocks, C. J. Kaalund, F. Ladouceur, S. T. Huntington, B. C. Gibson, S. Trpkovski, D. Simpson, E. Ampem-Lassen, S. Prawer, J. E. Butler, Reactive ion etching of waveguide structures in diamond, Diamond and Related Materials 17 (11) (2008) 1831-1834. doi:10.1016/j.diamond.2008.04.019.

[189] M. P. Hiscocks, K. Ganesan, B. C. Gibson, S. T. Huntington, F. Ladouceur, S. Prawer, Diamond waveguides fabricated by reactive ion etching, Optics Express 16 (24) (2008) 19512. doi:10.1364/OE.16.019512.

[190] A. Faraon, C. Santori, Z. Huang, K.-M. C. Fu, V. M. Acosta, D. Fattal, R. G. Beausoleil, Quantum photonic devices in single-crystal diamond, New Journal of Physics 15 (2) (2013) 025010. doi:10.1088/13672630/15/2/025010.

[191] B. J. M. Hausmann, I. B. Bulu, P. B. Deotare, M. McCutcheon, V. Venkataraman, M. L. Markham, D. J. Twitchen, M. Lončar, Integrated High-Quality Factor Optical Resonators in Diamond, Nano Letters 13 (5) (2013) 18981902. doi:10.1021/n13037454.

[192] K.-M. C. Fu, C. Santori, P. E. Barclay, I. Aharonovich, S. Prawer, N. Meyer, a. M. Holm, R. G. Beausoleil, Coupling of nitrogen-vacancy centers in diamond to a GaP waveguide, Applied Physics Letters 93 (23) (2008) 234107. doi:10.1063/1.3045950.

[193] P. E. Barclay, K.-M. C. Fu, C. Santori, R. G. Beausoleil, Chip-based microcavities coupled to nitrogen-vacancy centers in single crystal diamond, Applied Physics Letters 95 (19) (2009) 191115. doi:10.1063/1.3262948.

[194] P. E. Barclay, K.-m. Fu, C. Santori, R. G. Beausoleil, Hybrid photonic crystal cavity and waveguide for coupling to diamond NV-centers, Optics Express 17 (12) (2009) 9588. arXiv:arXiv:0901.3092v2, doi:10.1364/OE.17.009588.

[195] C. Santori, P. E. Barclay, K.-M. C. Fu, R. G. Beausoleil, S. Spillane, M. Fisch, Nanophotonics for quantum optics using nitrogen-vacancy centers in diamond, Nanotechnology 21 (27) (2010) 274008. doi:10.1088/09574484/21/27/274008

[196] K.-M. C. Fu, P. E. Barclay, C. Santori, A. Faraon, R. G. Beausoleil, Low-temperature tapered-fiber probing of diamond nitrogen-vacancy ensembles coupled to GaP microcavities, New Journal of Physics 13 (5) (2011) 055023. doi:10.1088/1367-2630/13/5/055023

[197] N. Thomas, R. J. Barbour, Y. Song, M. L. Lee, K.-m. C. Fu, Waveguide-integrated single-crystalline GaP resonators on diamond, Optics Express 22 (11) (2014) 13555. doi:10.1364/OE.22.013555

[198] M. Gould, S. Chakravarthi, I. R. Christen, N. Thomas, S. Dadgostar, Y. Song, M. L. Lee, F. Hatami, K.-M. C. Fu, Large-scale GaP-on-diamond integrated photonics platform for NV center-based quantum information, Journal of the Optical Society of America B 33 (3) (2016) B35. arXiv:1510.05047, doi:10.1364/JOSAB.33.000B35.

[199] M. Gould, E. R. Schmidgall, S. Dadgostar, F. Hatami, K.-M. C. Fu, Efficient Extraction of Zero-Phonon-Line Photons from Single Nitrogen-Vacancy Centers in an Integrated GaP-on-Diamond Platform, Physical Review Applied 6 (1) (2016) 011001. arXiv:1606.01826, doi:10.1103/PhysRevApplied.6.011001

[200] B. J. M. Hausmann, B. J. Shields, Q. Quan, Y. Chu, N. P. de Leon, R. Evans, M. J. Burek, a. S. Zibrov, M. Markham, D. J. Twitchen, H. Park, M. D. Lukin, M. Loncr, Coupling of NV Centers to Photonic Crystal Nanobeams in Diamond, Nano Letters 13 (12) (2013) 5791-5796. doi:10.1021/n1402174g.

[201] I. Bayn, S. Mouradian, L. Li, J. A. Goldstein, T. Schr??der, J. Zheng, E. H. Chen, O. Gaathon, M. Lu, A. Stein, 
C. A. Ruggiero, J. Salzman, R. Kalish, D. Englund, Fabrication of triangular nanobeam waveguide networks in bulk diamond using single-crystal silicon hard masks, Applied Physics Letters 105 (21) (2014) 2012-2017. arXiv:1411.3639, doi:10.1063/1.4902562.

[202] B. Khanaliloo, M. Mitchell, A. C. Hryciw, P. E. Barclay, High- $i i_{i} Q_{i} / i_{i} / i_{i} V_{i} / i_{i}$ Monolithic Di amond Microdisks Fabricated with Quasi-isotropic Etching, Nano Letters 15 (8) (2015) 5131-5136. doi:10.1021/acs.nanolett.5b01346.

URL http://pubs.acs.org/doi/abs/10.1021/acs.nanolett.5b01346

[203] B. Khanaliloo, H. Jayakumar, A. C. Hryciw, D. P. Lake, H. Kaviani, P. E. Barclay, Single-Crystal Diamond Nanobeam Waveguide Optomechanics, Physical Review X 5 (4) (2015) 041051. arXiv:1502.01788, doi:10.1103/PhysRevX.5.041051.

[204] K. J. Vahala, Optical microcavities, Nature 424 (6950) (2003) 839-846. doi:10.1038/nature01939. URL http: //www . nature.com/doifinder/10.1038/nature01939

[205] A. Imamoglu, D. D. Awschalom, G. Burkard, D. P. DiVincenzo, D. Loss, M. Sherwin, A. Small, Quantum Information Processing Using Quantum Dot Spins and Cavity QED, Physical Review Letters 83 (20) (1999) 42044207. arXiv:9904096v2, doi:10.1103/PhysRevLett.83.4204. URL http://link.aps.org/doi/10.1103/PhysRevLett.83.4204

[206] Z. Di, H. V. Jones, P. R. Dolan, S. M. Fairclough, M. B. Wincott, J. Fill, G. M. Hughes, J. M. Smith, Controlling the emission from semiconductor quantum dots using ultra-small tunable optical microcavities, New Journal of Physics 14 (10) (2012) 103048. doi:10.1088/1367-2630/14/10/103048.

[207] J. Wolters, A. W. Schell, G. Kewes, N. Nusse, M. Schoengen, H. Doscher, T. Hannappel, B. Lochel, M. Barth O. Benson, Enhancement of the zero phonon line emission from a single nitrogen vacancy center in a nanodiamond via coupling to a photonic crystal cavity, Applied Physics Letters 97 (14) (2010) 141108. doi:10.1063/1.3499300.

[208] D. Englund, B. Shields, K. Rivoire, F. Hatami, J. Vuckovic, H. Park, M. D. Lukin, Deterministic Coupling of a Single Nitrogen Vacancy Center to a Photonic Crystal Cavity, Nano Letters 10 (10) (2010) 3922-3926. doi:10.1021/nl101662v

[209] T. van der Sar, E. C. Heeres, G. M. Dmochowski, G. de Lange, L. Robledo, T. H. Oosterkamp, R. Hanson, Nanopositioning of a diamond nanocrystal containing a single nitrogen-vacancy defect center, Applied Physics Letters 94 (17) (2009) 173104. doi:10.1063/1.3120558.

URL http://link.aip.org/link/APPLAB/v94/i17/p173104/s1\&Agg=doi

[210] I. Bayn, J. Salzman, Ultra high-Q photonic crystal nanocavity design: The effect of a low- $\epsilon$ slab material, Optics Express 16 (7) (2008) 4972. arXiv:0802.3342, doi:10.1364/OE.16.004972.

[211] S. Tomljenovic-Hanic, M. J. Steel, C. M. de Sterke, J. Salzman, Diamond based photonic crystal microcavities, Optics Express 14 (8) (2006) 3556. doi:10.1364/OE.14.003556.

[212] S. Mosor, J. Hendrickson, B. C. Richards, J. Sweet, G. Khitrova, H. M. Gibbs, T. Yoshie, A. Scherer, O. B. Shchekin, D. G. Deppe, Scanning a photonic crystal slab nanocavity by condensation of xenon, Applied Physics Letters 87 (14) (2005) 141105. doi:10.1063/1.2076435.

[213] Q. Quan, P. B. Deotare, M. Loncar, Photonic crystal nanobeam cavity strongly coupled to the feeding waveguide, Applied Physics Letters 96 (20) (2010) 203102. arXiv:1002.1319, doi:10.1063/1.3429125.

[214] T. G. Tiecke, K. P. Nayak, J. D. Thompson, T. Peyronel, N. P. de Leon, V. Vuletić, M. D. Lukin, Efficient fiber-optical interface for nanophotonic devices, Optica 2 (2) (2015) 70. arXiv:arXiv:1409.7698v1, doi:10.1364/OPTICA.2.000070

[215] R. N. Patel, T. Schröder, N. Wan, L. Li, S. L. Mouradian, E. H. Chen, D. R. Englund, Efficient photon coupling from a diamond nitrogen vacancy center by integration with silica fiber, Light: Science \& Applications 5. doi:10.1038/1sa.2016.32.

[216] Y.-S. Park, A. K. Cook, H. Wang, Cavity QED with diamond nanocrystals and silica microspheres., Nano letters 6 (9) (2006) 2075-9. doi:10.1021/n1061342r.

URL http://www.ncbi.nlm.nih.gov/pubmed/16968028

[217] S. Schietinger, T. Schröder, O. Benson, One-by-one coupling of single defect centers in nanodiamonds to high-Q modes of an optical microresonator., Nano letters 8 (11) (2008) 3911-5. doi:10.1021/n18023627. URL http://www.ncbi.nlm.nih.gov/pubmed/18811213

[218] M. Larsson, K. N. Dinyari, H. Wang, Composite Optical Microcavity of Diamond Nanopillar and Silica Microsphere, Nano Letters 9 (4) (2009) 1447-1450. doi:10.1021/n18032944.

[219] P. E. Barclay, C. Santori, K.-M. Fu, R. G. Beausoleil, O. Painter, Coherent interference effects in a nanoassembled diamond NV center cavity-QED system, Optics Express 17 (10) (2009) 8081. arXiv:0812.4505, doi:10.1364/OE.17.008081.

[220] M. Gregor, R. Henze, T. Schroder, O. Benson, On-demand positioning of a preselected quantum emitter on a fiber-coupled toroidal microresonator, Applied Physics Letters 95 (15) (2009) 153110. doi:10.1063/1.3243989.

[221] R. J. Barbour, K. N. Dinyari, H. Wang, A composite microcavity of diamond nanopillar and deformed silica microsphere with enhanced evanescent decay length, Optics Express 18 (18) (2010) 18968. arXiv:1001.5449, 
doi:10.1364/OE. 18.018968

[222] B. Khanaliloo, M. Mitchell, A. C. Hryciw, P. E. Barclay, High- Q / V Monolithic Diamond Microdisks Fabricated with Quasi-isotropic Etching, Nano Letters 15 (8) (2015) 5131-5136. doi:10.1021/acs.nanolett.5b01346.

[223] S. Schwarz, S. Dufferwiel, P. M. Walker, F. Withers, a. a. P. Trichet, M. Sich, F. Li, E. a. Chekhovich, D. N. Borisenko, N. N. Kolesnikov, K. S. Novoselov, M. S. Skolnick, J. M. Smith, D. N. Krizhanovskii, a. I. Tartakovskii, Two-Dimensional MetalChalcogenide Films in Tunable Optical Microcavities, Nano Letters 14 (12) (2014) 7003-7008. arXiv:1408.3612, doi:10.1021/n1503312x.

[224] D. Hunger, T. Steinmetz, Y. Colombe, C. Deutsch, T. W. Hänsch, J. Reichel, A fiber FabryPerot cavity with high finesse, New Journal of Physics 12 (6) (2010) 065038. doi:10.1088/1367-2630/12/6/065038.

[225] G. Cui, J. M. Hannigan, R. Loeckenhoff, F. M. Matinaga, M. G. Raymer, S. Bhongale, M. Holland, S. Mosor, S. Chatterjee, H. M. Gibbs, G. Khitrova, A hemispherical, high-solid-angle optical micro-cavity for cavity-QED studies, Optics Express 14 (6) (2006) 2289. arXiv:0601046, doi:10.1364/OE.14.002289.

[226] T. Steinmetz, Y. Colombe, D. Hunger, T. W. Hansch, A. Balocchi, R. J. Warburton, J. Reichel, Stable fiber-based Fabry-Perot cavity, Applied Physics Letters 89 (11) (2006) 111110. arXiv:0606231v1, doi:10.1063/1.2347892.

[227] M. Trupke, E. a. Hinds, S. Eriksson, E. A. Curtis, Z. Moktadir, E. Kukharenka, M. Kraft, Microfabricated high-finesse optical cavity with open access and small volume, Applied Physics Letters 87 (21) (2005) 211106 doi: $10.1063 / 1.2132066$

[228] A. Muller, E. B. Flagg, M. Metcalfe, J. Lawall, G. S. Solomon, Coupling an epitaxial quantum dot to a fiber-based external-mirror microcavity, Applied Physics Letters 95 (17) (2009) 173101. arXiv:0910.4658, doi:10.1063/1.3245311.

[229] D. Hunger, C. Deutsch, R. J. Barbour, R. J. Warburton, J. Reichel, Laser micro-fabrication of concave, lowroughness features in silica, AIP Advances 2 (1) (2012) 012119. arXiv:1109.5047, doi:10.1063/1.3679721.

[230] L. Greuter, S. Starosielec, D. Najer, A. Ludwig, L. Duempelmann, D. Rohner, R. J. Warburton, A small mode volume tunable microcavity: Development and characterization, Applied Physics Letters 105 (12) (2014) 121105 arXiv:1408.1357, doi:10.1063/1.4896415.

[231] D. Najer, M. Renggli, D. Riedel, S. Starosielec, R. J. Warburton, Fabrication of mirror templates in silica with micron-sized radii of curvature, Applied Physics Letters 110 (1) (2017) 011101. arXiv:1608.04527, doi: $10.1063 / 1.4973458$.

[232] P. R. Dolan, G. M. Hughes, F. Grazioso, B. R. Patton, J. M. Smith, Femtoliter tunable optical cavity arrays, Optics Letters 35 (21) (2010) 3556. doi:10.1364/OL.35.003556.

[233] A. a. P. Trichet, P. R. Dolan, D. M. Coles, G. M. Hughes, J. M. Smith, Topographic control of openaccess microcavities at the nanometer scale, Optics Express 23 (13) (2015) 17205. arXiv:arXiv:1502.01532, doi:10.1364/OE.23.017205.

[234] J. F. S. Brachmann, H. Kaupp, T. W. Hänsch, D. Hunger, Photothermal effects in ultra-precisely stabilized tunable microcavities, Optics Express 24 (18) (2016) 21205-21215.

[235] R. Fechner, D. Flamm, W. Frank, A. Schindler, F. Frost, B. Ziberi, Ion beam assisted smoothing of optical surfaces, Applied Physics A: Materials Science \& Processing 78 (5) (2004) 651-654. doi:10.1007/s00339-003-2274-6.

[236] S. Johnson, P. R. Dolan, T. Grange, A. A. P. Trichet, G. Hornecker, Y. C. Chen, L. Weng, G. M. Hughes, A. A. R. Watt, A. Auffèves, J. M. Smith, Tunable cavity coupling of the zero phonon line of a nitrogenvacancy defect in diamond, New Journal of Physics 17 (12) (2015) 122003. arXiv:1506.05161, doi:10.1088/13672630/17/12/122003.

[237] H. Kaupp, C. Deutsch, H.-C. Chang, J. Reichel, T. W. Hänsch, D. Hunger, Scaling laws of the cavity enhancement for nitrogen-vacancy centers in diamond, Physical Review A 88 (5) (2013) 053812. arXiv:1304.0948, doi:10.1103/PhysRevA.88.053812.

[238] R. Albrecht, A. Bommer, C. Deutsch, J. Reichel, C. Becher, Coupling of a Single Nitrogen-Vacancy Center in Diamond to a Fiber-Based Microcavity, Physical Review Letters 110 (24) (2013) 243602. arXiv:arXiv:1303.7418v2, doi:10.1103/PhysRevLett.110.243602.

[239] R. Albrecht, A. Bommer, C. Pauly, F. Mücklich, A. W. Schell, P. Engel, T. Schröder, O. Benson, J. Reichel, C. Becher, Narrow-band single photon emission at room temperature based on a single nitrogen-vacancy center coupled to an all-fiber-cavity, Applied Physics Letters 105 (7) (2014) 073113. doi:10.1063/1.4893612.

[240] H. Kaupp, T. Hümmer, M. Mader, B. Schlederer, J. Benedikter, P. Haeusser, H.-C. Chang, H. Fedder, T. W. Hänsch, D. Hunger, Purcell-enhanced single-photon emission from nitrogen-vacancy centers coupled to a tunable microcavity, ArXiv 1 (2016) 1-8. arXiv:1606.00167.

[241] J. Benedikter, H. Kaupp, T. Hümmer, Y. Liang, A. Bommer, C. Becher, A. Krueger, J. M. Smith, T. W. Hänsch, D. Hunger, A cavity-enhanced single photon source based on the silicon vacancy center in diamond (2016) 1 6arXiv: 1612.05509.

[242] P. Andrich, B. J. Alemán, J. C. Lee, K. Ohno, C. F. de las Casas, F. J. Heremans, E. L. Hu, D. D. Awschalom, Engineered Micro- and Nanoscale Diamonds as Mobile Probes for High-Resolution Sensing in Fluid, Nano Letters 14 (9) (2014) 4959-4964. doi:10.1021/n1501208s. 
[243] H. S. Knowles, D. M. Kara, M. Atatüre, Observing bulk diamond spin coherence in high-purity nanodiamonds, Nature Materials 13 (1) (2013) 21-25. arXiv:1310.1133, doi:10.1038/nmat3805.

[244] M. E. Trusheim, L. Li, A. Laraoui, E. H. Chen, H. Bakhru, T. Schröder, O. Gaathon, C. a. Meriles, D. Englund, Scalable Fabrication of High Purity Diamond Nanocrystals with Long-Spin-Coherence Nitrogen Vacancy Centers, Nano Letters 14 (1) (2014) 32-36. doi:10.1021/n1402799u.

[245] Y. Shen, T. Sweeney, H. Wang, Zero-phonon linewidth of single nitrogen vacancy centers in diamond nanocrystals, Physical Review B 77 (3) (2008) 033201. doi:10.1103/PhysRevB.77.033201

[246] E. Janitz, M. Ruf, M. Dimock, A. Bourassa, J. Sankey, L. Childress, Fabry-Perot microcavity for diamond-based photonics, Physical Review A 92 (4) (2015) 043844. arXiv:1508.06588, doi:10.1103/PhysRevA.92.043844.

[247] S. Bogdanovic, S. B. van Dam, C. Bonato, L. C. Coenen, A.-m. J. Zwerver, B. Hensen, M. S. Z. Liddy, T. Fink, A. Reiserer, M. Loncar, R. Hanson, Design and low-temperature characterization of a tunable microcavity for diamond-based quantum networks (2016) 1-5arXiv:1612.02164. 